\title{
Survey of an approach to quantum measurement, classical properties and realist interpretation problems
}

\author{
P. Hájíček \\ Institute for Theoretical Physics \\ University of Bern \\ Sidlerstrasse 5, CH-3012 Bern, Switzerland \\ hajicek@itp.unibe.ch \\ and \\ Jiří Tolar \\ Department of Physics \\ Faculty of Nuclear Sciences and Physical Engineering \\ Czech Technical University in Prague \\ Břehová 7 \\ 11519 Prague, Czech Republic
}

December 2010

PACS number: 03.65.Ta,03.65.-w,03.65.Ca 


\begin{abstract}
The paper gives a systematic review of the basic ideas of (non-relativistic) quantum mechanics including all improvements that result from previous work of the authors. The aim is to show that the new theory is self-consistent and that it reproduces all measurable predictions of standard quantum mechanics. The most important improvements are: 1) A new realist interpretation of quantum mechanics assumes that all properties that are uniquely determined by preparations can be viewed as objective. These properties are then sufficient to justify the notion of quantum object. 2) Classical systems are defined as macroscopic quantum objects in states close to maximum entropy. For classical mechanics, new states of such kind are introduced, the so-called maximum-entropy packets, and shown to approximate classical dynamics better than Gaussian wave packets. 3) A new solution of quantum measurement problem is proposed for measurements that are performed on microsystems. First, it is assumed that readings of registration apparatuses are always signals from detectors. Second, an application of the cluster separability principle leads to a locality requirement on observables and to the key notion of separation status. Separation status of a microsystem is shown to change in preparation and registration processes. This gives preparation and registration new meaning and enhances the importance of these notions. Changes of separation status are alterations of kinematic description rather than some parts of dynamical trajectories and thus more radical than 'collapse of the wave function'. Standard quantum mechanics does not provide any information of how separation status changes run, hence new rules must be formulated. As an example of such a new rule, Beltrametti-Cassinelli-Lahti model of measurement is modified and shown then to satisfy both the probability-reproducibility and the objectification requirements.
\end{abstract}




\section{Contents}

0.1 Introduction . . . . . . . . . . . . . . . 3

0.1 .1 Examples of quantum systems . . . . . . . . . . . . 4

0.1 .2 Examples of quantum experiments . . . . . . . . . 6

0.1 .3 Realism . . . . . . . . . . . . . . . . . . 10

I General notions and rules $\quad 15$

1 States and observables 17

1.1 States . . . . . . . . . . . . . . . . . . . . 17

1.1.1 Mathematical preliminaries . . . . . . . . . . . . 18

1.1 .2 General rules . . . . . . . . . . . . . . . . . . 22

1.2 Observables ................................. 25

1.2.1 Mathematical preliminaries . . . . . . . . . . . 26

1.2 .2 General rules . . . . . . . . . . . . . . . . . . . 28

1.3 Galilean group $\ldots \ldots \ldots \ldots \ldots \ldots \ldots$

1.3.1 Euclidean group . . . . . . . . . . . . . . . . . . . 34

1.3.2 Time translations . . . . . . . . . . . . . . . . 36

2 Composition of quantum systems

2.1 Composition of systems of different types . . . . . . . . . . . . . 40

2.1.1 Tensor product of Hilbert spaces . . . . . . . . . . . . . . 40

2.1 .2 Entanglement . . . . . . . . . . . . . . . . . . 43

2.2 Composition of identical systems . . . . . . . . . . . 46

2.2 .1 Identical subsystems . . . . . . . . . . . . . . . . . . 46

2.2 .2 Cluster separability . . . . . . . . . . . . . . . . . 49

$2.2 .3 \quad$ D-local states and observables . . . . . . . . . . . . 52

2.2 .4 Separation status . . . . . . . . . . . . . . . 57

3 Further general ideas 61

3.1 Probability and information . . . . . . . . . . . 61

3.2 Joint measurability and contextuality . . . . . . . . . . 65 
3.2 .1 Joint measurability . . . . . . . . . . . . . . . . . 65

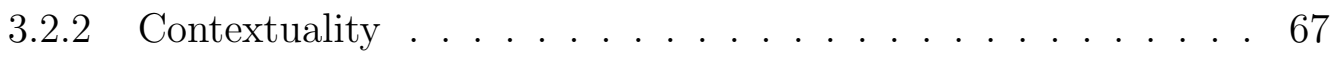

3.3 Superselection rules . . . . . . . . . . . . . . . . . . . 68

II The models

4 Models of microscopic systems 74

4.1 Basic PV measures . . . . . . . . . . . . . . . 75

4.2 Hamiltonian . . . . . . . . . . . . . . . . . 77

4.3 Galilean group . . . . . . . . . . . . . . . . 78

4.4 Joint measurement of position

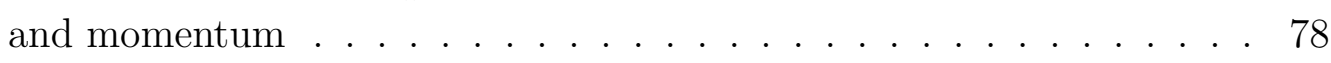

5 Quantum models of classical properties 83

5.1 Modified correspondence principle . . . . . . . . . . . 84

5.2 A model of thermodynamic properties . . . . . . . . 88

5.2.1 Composition, Hamiltonian and spectrum . . . . . . . . 88

5.2 .2 The length of the body . . . . . . . . . . . . . . . . 91

5.3 Maximum entropy assumption in classical mechanics . . . . . . . . . . . . . . . . . . . 92

5.4 Classical ME packets . . . . . . . . . . . . . . 94

5.4 .1 Definition and properties . . . . . . . . . . . 95

5.4 .2 Classical equations of motion . . . . . . . . . . . 96

5.5 Quantum ME packets . . . . . . . . . . . . . . . . . 97

5.5.1 Definition and properties . . . . . . . . . . . . . . 98

5.5.2 Quantum equations of motion . . . . . . . . . . . 999

5.6 Classical limit . . . . . . . . . . . . . . . . . . . . . 100

6 Quantum models of preparation and registration of microsystems 103

6.1 Beltrametti-Cassinelli-Lahti model . . . . . . . . . . . . . . . 104

6.1.1 Repeatable premeasurement and von Neumann model . . . . . 107

6.2 Comparison with real experiment.

Importance of detectors . . . . . . . . . . . . . . . 109

6.3 A simple modification of BCL model . . . . . . . . . . . 115

6.3.1 Proofs of Propositions 22 and $23 \ldots \ldots \ldots$

7 Conclusion $\quad \mathbf{1 2 4}$ 


\subsection{Introduction}

Since its beginnings, quantum mechanics has been plagued by two often discussed paradoxes. The first one, let us call it 'non-objectivity-of-the measurable', is that the values obtained by most measurements on microsystems cannot be assumed to exist before the measurements, that is, to be objective properties of the microsystem on which the measurement is done. An assumption of this kind of objectivity would lead to contradictions under various circumstances (contextuality [7, 40], Bell inequalities [4], Hardy impossibilities [33], Greenberger-Horne-Zeilinger equality [27]). This seduces people into thinking that quantum systems are 'elusive' [56] or 'not physical objects' [48].

The second one, let us call it 'Schrödinger-cat' paradox, is that application of quantum mechanics to interactions between microscopic and macroscopic systems leads to results that contradict experience (if artificial augmentations of the theory such as 'collapse of the wave function' are forbidden). Indeed, the result often predicted by quantum mechanics for such an interaction is that the macroscopic system evolves into a linear superposition of macroscopically different states (dead and alive cat). This also underlies the problem of quantum measurement: the state of a macroscopic apparatus after a measurement as predicted by quantum mechanics does not satisfy the objectification requirement [16]. According to the requirement, a well-defined value can be read off the apparatus after each individual measurement.

There is, however, another paradox that has as yet been left rather in shadow. Let us call it 'double-ruling' paradox: quantum mechanics has one set of rules for dealing with a microscopic system as if it were alone in the world (an isolated system) and another set of rules for it as a subsystem of a family of microsystems that are identical to it. To avoid contradictions resulting from the double ruling, the idea of cluster separability [56] has been invented. However, only short and superficial discussion of the idea within (non-relativistic) quantum mechanics seems to exist.

The present paper deals with the paradoxes. The non-objectivity-of-the-measurable is a fact of life but it itself only implies that some properties are not objective. Under certain conditions, microsystems have sufficient number of other properties that can be viewed as objective without contradictions [29]. Under these conditions, the microsystems can be considered as physical objects. This also helps to explain the emergence of classical properties [30]. The double-ruling and the Schrödingercat paradoxes are shown to be related. They can both be solved if the nature of cluster separability is clarified [31, 32]. The meaning and the role of preparations and registrations appear then in a new light. In any case, the quantum mechanics emerging on the basis of our ideas is at many points different from the usual textbook version. This paper gives a short but systematic account of it in order to show that it is a coherent theory free from internal contradictions. 
Any physical theory can be divided into two parts. First, there are basic notions and rules that form a kind of language. The language is general so that it is, on the one hand, very broadly applicable, and, on the other, unable to make any specific numerical predictions. Thus, it is difficult - may be even impossible - to disprove by some observational facts. Second, there are models formulated with the help of the language that are to explain and predict properties of observed systems. Each model introduces assumptions about the structure of the system and values of various parameters so that quantitative results can be obtained. These can be disproved. For example, in Newtonian mechanics, the notion of state as a point in phase space, of forces, the law of gravitation and Newton's dynamical equations can form the language. To describe the solar system, assumptions such as the number of bodies, their masses and certain class of their trajectories define a model. In the present paper, we attempt to separate quantum mechanics into the language and the model parts.

The idea that the main activity of theoreticians is to construct models of observed phenomena and that the general rules and notions must be adapted to this aim plays an important role in our approach. For example, many textbooks (see, e.g., Ref. [56]) maintain that any mathematically existing state of any quantum system can be prepared by some physical device, and that any mathematically existing observable of it can be registered by some physical device, without specifying the devices. The fact that such claims seem to be false for most macroscopic systems leads some authors (see, e.g., [48, 49]) to abandon the universality of quantum mechanics. However, quantum mechanics would work perfectly well without any of the two assumptions. On the one hand, one is free to use any mathematically existing state or observable for a model construction and for prediction of its properties. Mostly, only few are needed. On the other, it will turn out (see Secs. 2.2.2 and 6.2 ) that quantum analysis of the model assumptions itself implies that some of the mathematical observables cannot be registered.

\subsubsection{Examples of quantum systems}

To explain what is quantum mechanics about, this section mentions some known quantum systems. Thus, it belongs mainly to the model part of theory. However, it also introduces some general notions, such as microsystem, macrosystem, structural property or type of system.

Quantum mechanics is a theory that describes certain class of properties of certain class of systems in a similar way as any other physical theory does. For example, Newtonian mechanics describes bodies that can be considered as rigid in a good approximation and studies the motion of the bodies (we consider 'mass point' as an idealisation of a small rigid body). 
Quantum systems that we shall consider are photons, electrons, neutrons and nuclei, which we call particles, and systems containing some number of particles, such as atoms, molecules and macroscopic systems, which are called composite. Of course, neutrons and nuclei themselves are composite of quarks and gluons, but non-relativistic quantum mechanics can and must start from some phenomenological description of neutrons and nuclei. We just view them as structureless.

Let us call particles and quantum systems that are composite of small number of particles microsystems. They are extremely tiny and they can never be perceived directly by our senses. We can observe directly only macroscopic quantum systems that are composed of very many particles (except for photons: the eye can recognize signals of several photons). 'Very many' is: not too different from $10^{23}$ (the Avogadro number). Let us call these macrosystems. Some properties of most macroscopic systems obey classical theories. For example, shape and position of my chair belong to Euclidean geometry, chemical composition of its parts to classical chemistry and thermodynamic properties of the parts such as phase or temperature to phenomenological thermodynamics. Such properties are called 'classical'. Thus, properties of microsystems can only be observed via classical properties of macrosystems, if microsystems interact with them and this interaction leaves classical traces.

Microsystems are divided into types, such as electrons or hydrogen atoms. Systems of one type are not distinguishable from each other in a sense not existing in classical physics. Systems of the same type are often called identical. Microsystems exist always in a huge number of identical copies. The two properties of microsystems, viz. 1) their inaccessibility to direct observations and 2) utter lack of individuality that is connected with the existence of a huge number of identical copies, make them rather different from classical systems or 'things'. Each classical system can be observed directly by humans (in principle: for example, the distant galaxies) and each can be labelled and distinguished from other classical systems, because it is a quantum system composite of a huge number of particles and hence it is highly improbable that it has a relative of the same type in the world.

Objective properties that are common to all microsystems of the same type will be called structural. Thus, each particle has a mass, spin and electric charge. These are parameters that must be chosen in constructing models of existing systems taking into account their empirical values; they are not provided by the language part of theory. For example, each electron has a mass about .5 MeV, a spin 1/2 and a charge about $10^{-19} \mathrm{C}$. Any composite system consists of definite numbers of different particles with their masses, spins and charges. E.g., a hydrogen atom

\footnotetext{
${ }^{1}$ One can ask the question whether there is a non-relativistic limit of photons. In one such limit, photons may move with infinite velocity and their position need not therefore be very well defined. In another, photons may be represented by a classical electromagnetic wave.
} 
contains one electron and one proton (nucleus). The composition of a system is another structural property. The structural properties influence the dynamics of quantum systems; the way they do it and what dynamics is will be explained later. Only then, it will be clear what the meaning of these parameters is and that the type of each system can be recognized, if its dynamics is observed. When we shall know more about dynamics, further structural properties will emerge.

Structural properties are objective in the sense that they can be assumed to exist before and independently of any measurement. Such assumption does not lead to any contradictions with standard quantum mechanics and is at least tacitly made by most physicists. In fact, the ultimate goal of practically all experiments is to find structural properties of quantum systems.

From the formally logical point of view, all possible objective properties of given kind of objects ought to form a Boolean lattice. The structural properties satisfy this condition: systems with a given structural property form a subset of all systems. These subsets are always composed of whole type-classes of quantum systems. Clearly, the intersection of two such subsets and the complement of any such subset is again a structural property.

\subsubsection{Examples of quantum experiments}

The topic of this section plays an important role in understanding quantum mechanics. Specific examples of typical experiments will be given in some detail. That belongs to the model part of theory, but we gain access to some general notions of the language part such as preparation and registration. The concept of individual quantum object will be introduced and its relation to preparation and registration mentioned.

Let us first consider experiments with microsystems that are carried out in laboratories. Such an experiment starts at a source of microsystems that are to be studied. Let us give examples of sources for each microsystem.

1. Electrons. One possible source (called field emission, see e.g. Ref. [52], p. 38) consists of a cold cathode in the form of a sharp tip and a flat anode with an aperture in the middle at some distance from the cathode, in a vacuum tube. The electrostatic field of, say, few $\mathrm{kV}$ will enable electrons to tunnel from the metal and form an electron beam of about $10^{7}$ electrons per second through the aperture, with a relatively well-defined energy.

2. Neutrons can be obtained through nuclear reaction. This can be initiated by charged particles or gamma rays that can be furnished by an accelerator or a radioactive substance. For example the so-called Ra-Be source consists of finely divided $\mathrm{RaCl}_{2}$ mixed with powdered Be, contained in a small capsule. 
The reaction is produced by the alpha particle of Ra. The yield for $1 \mathrm{mg} \mathrm{Ra}$ is about $10^{4}$ neutrons per second with broad energy spectrum from small energies to about $13 \mathrm{MeV}$. The emission of neutrons is roughly spherically symmetric centred at the capsule.

3. Atoms and molecules. A macroscopic specimen of the required substance in gaseous phase at certain temperature can be produced, e.g. by an oven. The gas is in a vessel with an aperture from which a beam of the atoms or molecules emerges.

One can easily observe that each source is completely determined by an arrangement of macroscopic bodies of different shapes, chemical compositions and temperatures, and that the electric or magnetic fields are determined by their macroscopic characteristics, such as field intensities: that is, by their classical properties. These properties determine uniquely what type of microsystem is produced. Let us call this description empirical. It is important that the classical properties defining a source do not include time and position so that the source can be reproduced later and elsewhere. We call different sources that are defined by the same classical properties equivalent. Empirical description is sufficient for reproducibility of experiments but it is not sufficient for understanding of how the sources work. If a source defined by an empirical description is set into action, we have an instance of the so-called preparation.

Quantum mechanics assumes that these are general features of all sources, independently of whether they are arranged in a laboratory by humans or occur spontaneously in nature. For example, some classical conditions in the centre of the Sun lead to emission of neutrinos.

Often, a source yields very many microsystems of a given type that are emitted in all possible directions, a kind of radiation. We stress that the detailed structure of the radiation as it is understood in classical physics, that is where each individual classical system exactly is at different times, is not determined for quantum systems and the question has even no sense. Still, a fixed source gives the microsystems that originate from it some properties. In quantum mechanics, these properties are described on the one hand by the structural properties that define the prepared type, on the other, e.g., by the so-called quantum state. We call a prepared quantum state quantum object. One preparation creates one individual quantum object. However, a statement about a quantum object make sense only if it is independent of the individuality of the quantum microsystem that occupies the corresponding state.

To determine the quantum state that results from a preparation with a given empirical description in each specific case requires the full formalism of quantum mechanics. Hence, we must postpone this point. 
After arranging the source, another stage of the experiment can start. Generally, only a very small part of the radiation from a source has the properties that are needed for the planned experiment. The next step is, therefore, to select the part and to block off the rest. This is done by the so-called collimator, mostly a set of macroscopic screens with apertures and macroscopic electric or magnetic fields. For example, the electron radiation can go through an electrostatic field that accelerates the electrons and through electron-microscope 'lenses', each followed by a suitable screen. A narrow part of the original radiation, a beam, remains. For example, a beam of molecules obtained from an oven can also contain parts of broken molecules including molecules with different degrees of ionisation. The part with suitable composition can then be selected by a mass spectrometer and the rest blocked off by a screen. Again, the beam resulting from a raw source and a collimator consists of individual quantum objects with a well-defined type and quantum state. The process of obtaining these individual quantum objects is another example of preparation. Again, there is an empirical description that defines an equivalence class of preparations and equivalent preparations can be repeated.

The final beam can be characterized not only by the quantum state of individual objects but also by its approximate current, that is how many individual objects it yields per second. The beam can be made very thin. For example, in the electrondiffraction experiment [67], the beam that emerges from the collimator represents an electric current of about $10^{-16} \mathrm{~A}$, or $10^{3}$ electrons per second. As the approximate velocity of the electrons and the distance between the collimator and the detector are known, one can estimate the average simultaneous number of electrons there at each time. In the experiment, it is less than one. One can understand in this way that it is an experiment with individual electron objects.

Next, the beam can be lead through further arrangement of macroscopic bodies and fields. For example, to study the phenomenon of diffraction of electrons, each electron object can be scattered by a thin slab of crystalline graphite or by an electrostatic biprism interference apparatus. The latter consists of two parallel plates and a wire in between with a potential difference between the wire on the one hand and the plates on the other. An electron object runs through between the wire and both the left and right plate simultaneously and interferes with itself afterwards (for details see [67]). Again, the beam from the graphite or the biprism can be viewed as prepared by the whole arrangement of the source, collimator and the interference apparatus. This is another example of preparation procedure.

Finally, what results from the original beam must be made directly perceptible by its interaction with another system of macroscopic bodies and fields. This process is called registration and the system registration apparatus. The division of an experimental arrangement into preparation and registration parts is not unique. For 
example, in the electron-diffraction experiment, one example of a registration apparatus begins after the biprism interference, another one includes also the biprism interference apparatus. Similarly to preparations, the registrations are defined by an empirical description of their relevant classical properties in such a way that equivalent registrations can be repeated.

An important definition property of a registration is that it is applicable to an individual quantum object and that each empirical result of a registration is caused by just one individual object. In the above experiment, this assumption is made plausible by the extreme thinning of the beam, but it is adopted in general even if the beam is not thin.

An empirical description of a registration apparatus can determine a quantum mechanical observable. Again, more theory is needed for understanding of what are observables and how they are related to registration devices. Each individual registration performed by the apparatus, i.e., registration performed on a single quantum object, then gives some value of the observable. The registration is not considered to be finished without the registration apparatus having given a definite, macroscopic and classical response.

A part of registration apparatuses for microsystems are detectors. At the empirical level, a detector is determined by an arrangement of macroscopic fields and bodies, as well as by the chemical composition of its sensitive matter [45]. For example, in the experiment [67] on electron diffraction, the electrons coming from the biprism interference apparatus are absorbed in a scintillation film placed transversally to the beam. An incoming electron object is thus transformed into a light signal. The photons are guided by parallel system of fibres to a photo-cathode. The resulting (secondary) electrons are accelerated and lead to a micro-channel plate, which is a system of parallel thin photo-multipliers. Finally, a system of tiny anodes enables to record the time and the transversal position of the small flash of light in the scintillation film. In this way, each individual electron object coming from the biprism is detected at a position (two transversal coordinates determined by the anodes and one longitudinal coordinate determined by the position of the scintillation film). This triple of numbers is the result of each registration and the value of the corresponding observable, which is the position in this case. Also the time of the arrival at each anode can be approximately determined. Thus, each position obtains a certain time.

An important observation is the following. When an electron that has been prepared by the source and the collimator hits the scintillation film, it is lost as an object. Indeed, there is no property that would distinguish it from other electrons in the scintillation matter. Thus, this particular registration is a process inverse to preparation: while the preparation has created a quantum object with certain 
individuality, the registration entails a loss of the object. Our theory of quantum registration in Chap. 6 will generalise this observation.

If we repeat the experiment with individual electron objects many times and record the transversal position coordinates, the gradual formation of the electron interference pattern can be observed. The pattern can also be described by some numerical values. For example, the distance of adjacent maxima and the direction of the interference fringes can be such values. Still, the interference pattern is not a result of one but of a whole large set of individual registrations.

In some sense, each electron object must be spread out over the whole plane of the scintillation detector after coming from the biprism but the excitation of the molecules in the detector matter happens always only within a tiny well-localized piece of it, which is different for different objects. Thus, one can say that the interference pattern must be encoded in each individual electron object, even if it is not possible to obtain the property by a single registration. The interference pattern can be considered as an objective property of the individual electron objects prepared by the source, the collimator and the biprism interference apparatus. On the other hand, the hitting position of each individual electron object cannot be considered as its objective property. Such an assumption would lead to contradictions with results of other experiments. The position must be regarded as created in the detection process. The interference pattern is not a structural property: preparations that differ in the voltage at some stage of the experiment (e.g., the accelerating field in the collimator or the field between the wire and the side electrodes in the biprism interference apparatus, etc.) will give different interference patterns. We call such objective properties dynamical.

Some structural properties can be measured directly by a registration (on individual quantum objects) and their values are real numbers. For example, mass can be measured by a mass spectrometer. Such structural properties can be described by quantum observables (see Sec. 1.2)2. However, there are also structural properties that cannot be directly measured on individual objects similarly to the interference pattern, such as cross sections. They cannot be described by observables: the value of a cross section is not a value of an observable.

\subsubsection{Realism}

The fact that the description at empirical level is sufficient for reproducibility of experiments and for the account of their results has been used to construct a kind of realist interpretation of quantum mechanics. This interpretation maintains that

\footnotetext{
${ }^{2}$ These observables must commute with all other observables ([48], IV.8), and can be associated with the so-called superselection rules, see Sec. 3.3.
} 
the domain of real world to which quantum mechanics is applicable are exactly the classical properties of macroscopic devices described at the empirical level. A rigorous formulation is Ref. [48]. The macroscopic systems are not considered as quantum systems. Such interpretation does not even need to assume that there are any microsystems [49. This is in agreement with statements such as Bohr's 'there is no quantum concept', see Ref. [17], pp. 33-57.

There are two reasons for such a cautious approach. First, the values of observables concerning a quantum object $\mathcal{S}$ that are read off as the classical properties of a macroscopic device $\mathcal{A}$ after its interaction with the quantum object are not objective properties of the quantum object $\mathcal{S}$. If one assumes that such values are the only 'properties' any quantum systems can exhibit or that each objective property must be accessible by an individual registration, then quantum systems do not possess enough objective properties to be physical objects (for a mathematical proof, see Ref. [48, p. 60). Second, there is neither a completely satisfactory quantum theory of all classical properties of macrosystems nor a generally accepted understanding of quantum measurement process as yet.

The cautious approach has its drawbacks. The question naturally arises, what is the physics that prevents macroscopic systems to be quantum systems. After all, they are composite of quantum particles. The explanation offered in Refs. [48, 49] by G. Ludwig is as follows. Ludwig's method is to postulate certain statements on preparation and registration first and then to derive quantum mechanics from it. For such method to be successful, some completeness of the starting set of preparation and registration properties is needed. This, however, leads to problems: the macroscopic systems described by classical theories yield examples that do not admit such completeness (see also Chap. 5 of the present paper). Thus, if preparations and registrations are to form the domain of reality of a macroscopic system that is to be described by quantum mechanics, then quantum mechanics is not applicable (see Ref. 49], Vol. 2, p. 16). On the one hand, the argument underpins the idea that the cautious approach is self-consistent, on the other, it only shows that the approach itself is inapplicable to macroscopic systems and leaves the question open whether there is another more comprehensive approach.

These and other problems motivate our attempt at an alternative theory. Surprisingly enough, another realist interpretation that is compatible with all physical principles of standard quantum mechanics is possible. The only difficulty are certain prejudices and restrictions motivated, in fact, by classical mechanics. Thus, our deviation from classical thinking (with the exception of realism) is even greater than that of other interpretations of quantum mechanics.

The general hypothesis of realism says that the world is not just an idea in our 
mind but does really exist3. This is an utterly metaphysical hypothesis, which means that it cannot be disproved by any observational facts. Only if some further assumptions about the world are involved then it is indeed possible to prove in some cases that such a world does not exist.

Our approach to properties of quantum systems is necessarily different from those that can be found in literature in order that it can circumvent some no-go theorems concerning objective properties (see, e.g., Ref. [48]). First, we extend the notion of properties to include complex ones in the following sense:

1. Their values may be arbitrary mathematical objects (sets, maps between sets, etc.). For example, the Hamiltonian of a quantum system involves a relation between energy and some other observables of the system. This relation is an example of such a complex property.

2. Their values need not be directly obtained by individual registrations. For example, to measure a cross-section a whole series of scattering experiments must be done. Thus, their values need not possess probability distribution but may be equivalent to, or derivable from, probability distributions.

Second, we weaken the relation of objective properties to the basic quantum-mechanical concepts of preparation and registration. What is objective? A popular opinion is $(48]$, p. 60):

An objective property should refer directly to the microsystem itself and be independent of preparation and registration process. By this we mean that a set $a$ [of systems] which is selected by a preparation (and similarly a set $b$ which is selected by a registration) may be divided according to objective properties into subsets...

The 'independence' does not mean that properties are not manipulable or observable. In fact, if we look at Newton mechanics, a mass point can be put into a space point $\vec{x}$ with a momentum $\vec{p}$ so that it is then objectively in the state $(\vec{x}, \vec{p})$, and measurement procedures exist that can inform us in which state it objectively is in the moment of measurement. One can say that all classical objective properties can be prepared and can be obtained as results of measurements. The problem in quantum mechanics is that a registration in most cases disturbs the microsystem so that the result of the registration is only created during the registration process. The result of the registration cannot thus be assumed to refer directly to the microsystem and to be independent of the registration process. Hence, a set of prepared microsystem

\footnotetext{
${ }^{3}$ Of course, we are a part of the world and can influence it. Thus, the world is objective but not completely independent of us.
} 
objects cannot already be subdivided into subsets according to the properties that will only be registered later.

It is clear then that the objective properties of microsystems, if there are any, cannot be directly related to registrations. To confirm an objective property, many registration procedures often carried out by several registration devices must be done on many copies of equally prepared objects.

Any criterion of objectivity must therefore ignore registration. We are thus led to the following:

Basic Ontological Hypothesis of Quantum Mechanics A property is objective if its value is uniquely determined by a preparation according to the rules of standard quantum mechanics. The 'value' is the value of the mathematical expression that describes the property and it may be more general than just a real number. No registration is necessary to establish such a property but a correct registration cannot disprove its value; in many cases, registrations can confirm the value.

The motivation for the hypothesis is that such a property can be assumed to be possessed by the prepared object without either violating any rules of standard quantum mechanics or contradicting possible results of any registration performed on the prepared object. Any set of equally prepared objects can, therefore, always be subdivided into subsets according to such objective properties. It follows that the properties satisfy Boolean lattice rules. The relation of registrations to such objective properties must be studied for each property separately.

We shall discus the objective properties in more detail in Chap. 1 and see that they can be divided into structural and dynamical. Already now, we stress: with the help of the above criterion, we obtain enough objective properties to characterize quantum systems completely (at least from the standpoint of standard quantum mechanics). Moreover, there will be objective properties of quantum systems that can play the role of classical properties of macroscopic quantum systems.

A few words have to be said on ontological hypotheses. As it is well-known, the objective existence of anything cannot be proved (even that of the chair on which I am now sitting, see, e.g., Ref. [22], where this old philosophical tenet is explained from the point of view of a physicist). Thus, all such statements are only hypotheses, called ontological. However, an ontological hypothesis may lead to contradictions with some observations; exactly that happens if one tries to claim objectivity of quantum observables. Hypotheses that do not lead to contradictions may be useful. For example, the objective existence of the chair nicely explains why we all agree on its properties. Similarly, the assumption that quantum systems can possess some objective properties can be useful for the quantum theory of classical properties or for a solution of the problem of quantum measurement. The fact that a realist 
version of quantum mechanics appeals to our intuition is not essential, but it is a bonus. 


\section{Part I}

\section{General notions and rules}


As announced in the Introduction, we are going to divide quantum mechanics into two parts. The first one contains general notions and rules as well as the corresponding mathematical formalism and its physical interpretation, while the second is a menagerie of models. 


\section{Chapter 1}

\section{States and observables}

This chapter describes the first part of the general language of quantum mechanics concerning the notions and most important properties of states, observables and their relation to space-time transformations. We shall use some of the nomenclature introduced in Refs. [48, 16] but no previous knowledge of these books is assumed. Ref. [48] lists certain axioms first that must be satisfied by preparations and registrations and then derives quantum mechanics from these axioms. Our way will be the opposite one, similar to that of Ref. [16]: we assume the basic notions and rules of quantum mechanics first and derive general consequences for preparations and registrations later.

\section{$1.1 \quad$ States}

A preparation yields quantum object of certain type in a state while a registration results in a value of an observable. What is the type and what is the state is uniquely determined by the classical properties of the preparation and what is the observable is uniquely determined by the classical properties of the registration.

In quantum mechanics, the states and observables are described by specific mathematical entities. If we say 'state' or 'observable', we always mean these entities. It is important to distinguish between the fact of being uniquely determined and the way from the empirical description to a particular mathematical state or observable. The calculation of a state from classical conditions defined by the preparation needs full quantum mechanics. Similarly, to calculate an observable from classical properties of the registration device, quantum mechanics must be used. At this stage of exposition, we just assume that the classical properties determine states and observables and consider the way of how they do it as belonging to the model part of the theory. 


\subsubsection{Mathematical preliminaries}

We assume that the reader is already more or less acquainted with most of the necessary mathematics and the purpose of the following text is mainly to settle the notation.

Let $\mathbf{H}$ be a complex separable Hilbert space with inner product $\langle\cdot \mid \cdot\rangle$ satisfying $\langle a \phi \mid b \psi\rangle=a^{*} b\langle\phi \mid \psi\rangle$, where '*' denotes complex conjugation. An element $\phi \in \mathbf{H}$ is a unit vector if its norm defined by

$$
\|\phi\|=\langle\phi \mid \phi\rangle
$$

equals one,

$$
\|\phi\|=1
$$

and the non-zero vectors $\phi, \psi \in \mathbf{H}$ are orthogonal if

$$
\langle\phi \mid \psi\rangle=0
$$

A set $\left\{\phi_{k}\right\} \subset \mathbf{H}$ is orthonormal if the vectors $\phi_{k}$ are mutually orthogonal unit vectors. $\left\{\phi_{k}\right\} \subset \mathbf{H}, k=1,2, \cdots$, is an orthomormal basis of $\mathbf{H}$ if any $\psi \in \mathbf{H}$ can be expressed as a series

$$
\psi=\sum_{k}\left\langle\phi_{k} \mid \psi\right\rangle \phi_{k}
$$

with

$$
\|\psi\|^{2}=\sum_{k}\left|\left\langle\phi_{k} \mid \psi\right\rangle\right|^{2}
$$

Separability means that there is at least one countable basis.

Let $\mathbf{H}$ and $\mathbf{H}^{\prime}$ be two separable Hilbert spaces, $\left\{\phi_{k}\right\}$ and $\left\{\phi_{k}^{\prime}\right\}$ two orthonormal bases, $\left\{\phi_{k}\right\} \subset \mathbf{H}$ and $\left\{\phi_{k}^{\prime}\right\} \subset \mathbf{H}^{\prime}$. Let us define map $U:\left\{\phi_{k}\right\} \mapsto\left\{\phi_{k}^{\prime}\right\}$ by

$$
\mathrm{U} \phi_{k}=\phi_{k}^{\prime}
$$

for each $k$. Then, $\mathbf{U}$ can be extended by linearity and continuity to the whole of $\mathbf{H}$ and it maps $\mathbf{H}$ onto $\mathbf{H}^{\prime}$. The map $\mathbf{U}$ is called unitary. Unitary maps preserve linear superposition,

$$
\mathrm{U}(a \psi+b \phi)=a \mathrm{U} \psi+b \mathrm{U} \phi
$$

and inner product,

$$
\langle\mathrm{U} \psi \mid \mathrm{U} \phi\rangle=\langle\psi \mid \phi\rangle .
$$

They can be defined by these properties for general Hilbert spaces and used as equivalence morphisms in the theory of Hilbert spaces. Each two separable Hilbert spaces are thus unitarily equivalent. 
Any unit vector $\phi \in \mathbf{H}$ determines a one-dimensional (orthogonal) projection operator $\mathrm{P}[\phi]$ by the formula

$$
\mathrm{P}[\phi] \psi=\langle\phi \mid \psi\rangle \phi
$$

for all $\psi \in \mathbf{H}$. We also use the Dirac notation $|\phi\rangle\langle\phi|$ for this projector. If $\left\{\phi_{k}\right\}$ is an orthonormal basis of $\mathbf{H}$, then the projection operators $\mathrm{P}\left[\phi_{k}\right]$ satisfy

$$
\mathrm{P}\left[\phi_{k}\right] \mathrm{P}\left[\phi_{l}\right]=0
$$

for all $k \neq l$-we say they are mutually orthogonal-and

$$
\sum_{k} \mathrm{P}\left[\phi_{k}\right]=1,
$$

where 1 is the identity operator on $\mathbf{H}$.

A linear operator $\mathbf{A}: \mathbf{H} \mapsto \mathbf{H}$ is defined by the property

$$
\mathrm{A}(a \phi+b \psi)=a \mathrm{~A} \phi+b \mathrm{~A} \psi
$$

for all $\phi, \psi \in \mathbf{H}$ for which the right-hand side makes sense. A linear operator $\mathbf{A}$ is called bounded if its norm

$$
\|\mathrm{A}\|=\sup _{\|\psi\|=1}\|\mathrm{~A} \psi\|
$$

is finite. Let us denote the set of all bounded linear operators by $\mathbf{L}(\mathbf{H})$. Bounded linear operators can be multiplied,

$$
(\mathrm{AB}) \phi=\mathrm{A}(\mathrm{B} \phi),
$$

and linearly combined,

$$
(a \mathrm{~A}+b \mathrm{~B}) \phi=a(\mathrm{~A} \phi)+b(\mathrm{~B} \phi)
$$

for any $a, b \in \mathbb{C} . \mathbf{L}(\mathbf{H})$ is a Banach algebra with the norm (1.2).

The adjoint $A^{\dagger}$ of bounded operator $A$ is defined by

$$
\left\langle\mathrm{A}^{\dagger} \phi \mid \psi\right\rangle=\langle\phi \mid \mathrm{A} \psi\rangle
$$

for all $\phi, \psi \in \mathbf{H}$, and $\mathbf{A}$ is self-adjoint (s.a.) if

$$
\mathrm{A}^{\dagger}=\mathrm{A} \text {. }
$$

Linear combinations of bounded s.a. operators with real coefficients are again s.a., but their products are not, in general. Hence, the set of all bounded s.a. operators forms a real linear space. If completed with respect to the norm (1.2), it is a Banach space denoted by $\mathbf{L}_{r}(\mathbf{H})$. 
Unitary maps $\mathbf{U}: \mathbf{H} \mapsto \mathbf{H}$ are bounded operators and we obtain from Eq. (1.1)

$$
\mathrm{U}^{\dagger} \cdot \mathrm{U}=\mathrm{U} \cdot \mathrm{U}^{\dagger}=1
$$

Let $\mathbf{H}$ and $\mathbf{H}^{\prime}$ be two separable Hilbert spaces and $\mathbf{U}: \mathbf{H} \mapsto \mathbf{H}^{\prime}$ be a unitary map. Then $\mathbf{U}$ defines a map of $\mathbf{L}(\mathbf{H})$ onto $\mathbf{L}\left(\mathbf{H}^{\prime}\right)$ by $A \mapsto U A U^{\dagger}$. This map preserves operator action,

$$
\left(\mathrm{UAU}^{\dagger}\right)(\mathrm{U} \phi)=\mathrm{U}(\mathrm{A} \phi),
$$

linear relation,

$$
\mathrm{U}(a \mathrm{~A}+b \mathrm{~B}) \mathrm{U}^{\dagger}=a \mathrm{UAU}^{\dagger}+b \mathrm{UBU}^{\dagger},
$$

operator product

$$
\left(\mathrm{UAU}^{\dagger}\right)\left(\mathrm{UBU}^{\dagger}\right)=\mathrm{U}(\mathrm{AB}) \mathrm{U}^{\dagger}
$$

and norm,

$$
\|\mathrm{UA}\|=\|\mathrm{A}\|
$$

on $\mathbf{L}(\mathbf{H})$. The Banach-space structure of $\mathbf{L}_{r}(\mathbf{H})$ is also preserved by unitary maps.

An operator $\mathrm{A} \in \mathbf{L}_{r}(\mathbf{H})$ is positive, $\mathrm{A} \geq 0$, where 0 is the null operator, if

$$
\langle\phi \mid \mathrm{A} \phi\rangle \geq 0
$$

for all vectors $\phi \in \mathbf{H}$. The relation $\mathrm{A} \geq \mathrm{B}$ defined by

$$
A-B \geq 0
$$

is an ordering on this space. With this (partial) order relation, $\mathbf{L}_{r}(\mathbf{H})$ is an ordered Banach space. The order relation is preserved by unitary maps,

$$
\mathrm{UAU}^{\dagger} \geq \mathrm{UBU}^{\dagger} \quad \text { if } \mathrm{A} \geq \mathrm{B} .
$$

Let $\left\{\phi_{k}\right\}$ be any orthonormal basis of $\mathbf{H}$. For any $\mathbf{A} \in \mathbf{L}(\mathbf{H})$, we define the trace

$$
\operatorname{tr}[\mathrm{A}]=\sum_{k}\left\langle\phi_{k} \mid \mathrm{A} \phi_{k}\right\rangle .
$$

Trace is independent of basis and invariant with respect to unitary maps,

$$
\operatorname{tr}\left[\mathrm{UAU}^{\dagger}\right]=\operatorname{tr}[\mathrm{A}] .
$$

Proposition 1 Trace defines the norm $\|\mathrm{A}\|_{s}$ on $\mathbf{L}_{r}(\mathbf{H})$ by

$$
\|\mathrm{A}\|_{s}=\operatorname{tr}\left[\sqrt{\mathrm{A}^{2}}\right]
$$

satisfying

$$
\|\mathrm{A}\|_{s} \geq\|\mathrm{A}\|
$$

for all $\mathbf{A} \in \mathbf{L}_{r}(\mathbf{H})$ 
For proof, see Ref. [48], Appendix IV.11.

Definition 1 The norm (1.3) is called trace norm and all s.a. operators on $\mathbf{H}$ with finite trace norm are called trace-class. The set of all trace-class operators is denoted by $\mathbf{T}(\mathbf{H})$.

Trace norm is preserved by unitary maps.

Proposition $2 \mathbf{T}(\mathbf{H})$ completed with respect to the norm (1.3) is an ordered $B a-$ nach space. A trace-class operator is bounded, its trace is finite and its spectrum is discrete.

For proof, see Ref. [48], Appendix IV.11.

Let $\mathbf{T}(\mathbf{H})_{1}^{+}$be the set of all positive self-adjoint trace-class operators on $\mathbf{H}$ with trace 1 . As these operators are positive, their trace is equal to their trace norm and they lie on the unit sphere in $\mathbf{T}(\mathbf{H}) . \mathbf{T}(\mathbf{H})_{1}^{+}$is a convex set: let $\mathrm{T}_{1}, \mathrm{~T}_{2} \in \mathbf{T}(\mathbf{H})_{1}^{+}$, then

$$
w \mathrm{\top}_{1}+(1-w) \mathrm{T}_{2} \in \mathbf{T}(\mathbf{H})_{1}^{+}
$$

for all $0<w<1$. It follows that any convex combination

$$
\mathrm{\top}=\sum_{k} w_{k} \mathrm{\top}_{k}
$$

of at most countable set of states $\mathrm{T}_{k} \in \mathbf{T}(\mathbf{H})_{1}^{+}$with weights $w_{k}$ satisfying

$$
0 \leq w_{k} \leq 1, \quad \sum w_{k}=1
$$

and the series converging in the trace-norm topology also lies in $\mathbf{T}(\mathbf{H})_{1}^{+}$. In general, a given state $\mathbf{T}(\mathbf{H})_{1}^{+}$can be written in (infinitely) many ways as a convex combination. All possible components of such convex combinations form a so-called 'face' in the convex set $\mathbf{T}\left(\mathbf{H}_{\tau}\right)_{1}^{+}$(cf. [48], p. 75).

An element $\mathbf{T}$ is called extremal element of $\mathbf{T}(\mathbf{H})_{1}^{+}$if it lies in a zero-dimensional face, i.e., if the condition

$$
\mathrm{T}=w \mathrm{~T}_{1}+(1-w) \mathrm{T}_{2}
$$

with $\mathrm{T}_{1}, \mathrm{~T}_{2} \in \mathbf{T}(\mathbf{H})_{1}^{+}$and $0 \leq w \leq 1$, implies that $\mathrm{T}=\mathrm{T}_{1}=\mathrm{T}_{2}$. Any extremal element has the form $\mathrm{P}[\phi]$, where $\phi$ is a unit vector of $\mathbf{H}$ and, conversely, any projection onto a one-dimensional sub-space of $\mathbf{H}$ is extremal. The set of all extremal elements of $\mathbf{T}(\mathbf{H})_{1}^{+}$generates $\mathbf{T}(\mathbf{H})_{1}^{+}$in the sense that any $\mathbf{T} \in \mathbf{T}(\mathbf{H})_{1}^{+}$can be expressed as countable convex combination of some extremal elements,

$$
\mathrm{T}=\sum_{k} w_{k} \mathrm{P}\left[\phi_{k}\right]
$$


Such a decomposition can be obtained, in particular, from the spectral decomposition

$$
\mathrm{T}=\sum_{k} t_{k} \mathrm{P}_{k}
$$

In that case $\mathrm{T}$ is decomposed into mutually orthogonal one dimensional projectors $\mathrm{P}\left[\phi_{l}\right]$, with the eigenvalues representing the weights $w_{l}$, each term appearing as many times as given by the dimension of the eigenspace.

A unit vector $\phi$ of $\mathbf{H}$ defines a unique extremal element $\mathrm{P}[\phi] \in \mathbf{T}(\mathbf{H})_{1}^{+}$but $\mathrm{P}[\phi]$ determines $\phi$ only up to a phase factor $e^{i \alpha}$. Due to the (complex) linear structure of $\mathbf{H}$, there is an operation on vectors called linear superposition. Linear superposition $\psi=\sum c_{k} \phi_{k}$ of unit vectors with complex coefficients satisfying

$$
\sum_{k}\left|c_{k}\right|^{2}=1
$$

is another unit vector and the resulting projector $\mathbf{P}[\psi]$,

$$
\mathrm{P}[\psi]=\left|\sum_{k} c_{k} \phi_{k}\right\rangle\left\langle\sum_{l} c_{l} \phi_{l}\left|=\sum_{k l} c_{l}^{*} c_{k}\right| \phi_{k}\right\rangle\left\langle\left.\phi_{l}\left|\neq \sum_{k}\right| c_{k}\right|^{2} \mid \phi_{k}\right\rangle\left\langle\phi_{k}\right| .
$$

is different from the corresponding convex combination,

$$
\sum_{k}\left|c_{k}\right|^{2}\left|\phi_{k}\right\rangle\left\langle\phi_{k}\right|
$$

Observe that $\mathrm{P}[\psi]$ is not determined by the projectors $\mathrm{P}\left[\phi_{k}\right]$ because it depends on the relative phases of vectors $\phi_{k}$.

\subsubsection{General rules}

With each quantum system $\mathcal{S}$ of type $\tau$, a complex separable Hilbert space $\mathbf{H}_{\tau}$ is associated. $\mathbf{H}_{\tau}$ carries a further structure dependent on $\tau$, such as a representation of Galilean group (see Sec. 1.3) and a tensor-product structure (see Secs. 2.1 and $2.2)$.

Rule 1 The states of a quantum system $\mathcal{S}$ of type $\tau$ are elements of $\mathbf{T}\left(\mathbf{H}_{\tau}\right)_{1}^{+}$. Every preparation creates a quantum object of a definite type and a definite state. The state is an objective property of the prepared system. In principle, each element of $\mathbf{T}\left(\mathbf{H}_{\tau}\right)_{1}^{+}$ can be prepared as a state of a system of type $\tau$.

Textbooks of quantum mechanics do not consider states as objective but this assumption is crucial for our interpretation. The objectivity of states however does not mean that each system of type $\tau$ is in some state from $\mathbf{T}\left(\mathbf{H}_{\tau}\right)_{1}^{+}$even if it does 
not been so prepared (see Sec. 2.2.1). Rule 1 does not exclude that one and the same state can be obtained by different preparations. Methods of how states prepared by two different preparations can be compared will be described Sec. 1.2.

Definition 2 Let $\mathrm{T}$ be a finite convex combination (1.4). In the case that the preparation $\mathcal{P}[\mathrm{T}]$ of $\mathrm{T}$ includes sub-preparations $\mathcal{P}\left[\mathrm{T}_{k}\right]$ of each $\mathrm{T}_{k}$ with relative weights $w_{k}$ (independently of whether the mixing is done by humans or by nature; in practice, it is achieved by mixing beams), the decomposition (1.4) is selected by the preparation of $\mathrm{T}$. Let us call the resulting state gemenge, the states $\mathrm{T}_{k}$ components of the gemenge.

A gemenge is also called direct mixture [48] or proper mixture [22]; the word gemenge is taken from Ref. [16]. Gemenge concerns a physical property of preparation rather than any mathematical one of the right-hand side of Eq. (1.4) (such as $\mathrm{T}_{k}$ being vector states or being mutually orthogonal, etc). From the mathematical point of view, many different convex decompositions of a general state $\mathrm{T}$ may exist. $\mathrm{A}$ preparation of $\mathrm{T}$ selects one of the mathematically possible convex decompositions of $\mathrm{T}$.

A random mixture of preparations is not uniquely determined by the preparation process. It can be coarsened or refined, i.e., some of $\mathcal{P}\left[\mathrm{T}_{k}\right]$ can be combined into one preparation procedure or $\mathcal{P}\left[\mathrm{T}_{k}\right]$ for some $k$ can itself be a random mixture of other preparations $\mathcal{P}\left[\mathrm{T}_{k l}\right]$.

Definition 3 The finest convex decomposition of state $\mathrm{T}$ defined by its preparation as gemenge is called gemenge structure of $\mathrm{T}$.

Thus, gemenge structure of $\mathrm{T}$ is uniquely determined by its preparation. For extremal states, there is always only one gemenge structure, the trivial one, independently of how it was prepared.

It may be advantageous to distinguish the mathematical convex combination of states from their gemenge by writing the sum in Eq. (1.4) as follows

$$
\mathbf{T}=\left(\sum_{k=1}^{n}\right) w_{k} \mathbf{T}_{k}
$$

in the case that the right-hand side is a gemenge structure of $\mathrm{T}$.

A gemenge contains more information about an object than its state operator does and it is, therefore, viewed as a refinement of the state concept.

Proposition 3 The individual objects prepared in a state with gemenge structure (1.8) can be assumed always to objectively be in either of its component states $\mathrm{T}_{k}$ with respective probabilities $w_{k}$. 
It also follows that a state itself even if it is prepared as a gemenge with nontrivial structure (1.8) is an objective property of any individual object prepared in this way. On the one hand, this is in agreement with our objectivity criterion, on the other it is completely analogous to classical properties which need not determine the state of a classical system uniquely, either. The states and their gemenge structures are objective properties (being determined by preparations) that we have called dynamicall. We shall later encounter more examples of dynamical properties, but many will be derived from, or determined by, states.

Set $\mathbf{T}\left(\mathbf{H}_{\tau}\right)_{1}^{+}$of states can be viewed as analogous to phase space of Newtonian mechanics. Each point of $\mathbf{T}\left(\mathbf{H}_{\tau}\right)_{1}^{+}$is analogous to a point in the phase space if it is prepared so that it has a trivial gemenge structure. The gemenges are apparently analogous to the probability distributions over the phase space.

Let a classical system $\mathcal{S}_{c}$ be subject to physical conditions $\mathcal{C}$ that do not uniquely determine a point $Z$ in its phase space but a whole probability distribution $\rho(Z)$ on the phase space. Then, on the one hand, $\rho(Z)$ is an objective property of each individual system $\mathcal{S}_{c}$ subject to $\mathcal{C}$ and, on the other, each individual $\mathcal{S}_{c}$ is usually assumed also to be objectively at a particular point $Z$ (but cf. Chap. 5), which is, therefore, also its objective property. Thus, the two different states, $\rho(Z)$ and $Z$, are simultaneously objective properties of the particular system $\mathcal{S}_{c}$. This is, of course, possible, because the two properties are logically compatible.

The situation in quantum mechanics is completely analogous, but there is one difference. In Newtonian mechanics, point $Z$ and non-trivial distribution $\rho(Z)$ are mathematically different. In quantum mechanics, one and the same element $\mathrm{T} \in$ $\mathbf{T}\left(\mathbf{H}_{\tau}\right)_{1}^{+}$can have both a non-trivial and a trivial gemenge structure. This depends on its preparation. Mathematical properties of $\mathrm{T}$ can restrict its possible gemenge structures, e.g. extremal states allow only the trivial one.

Any unit vector $\phi \in \mathbf{H}_{\tau}$ defines state $\mathrm{P}[\phi]$. Such a state is called vector state (often, such states are called 'pure'). The states spoken about in Rule 1 are not necessarily vector states. However, vector states have a number of very interesting properties. In some special cases, vector states $\mathrm{P}\left[\phi_{k}\right], k=1, \cdots, N$, can be prepared in such a way that their relative phases are also fixed. Such set of states is called coherent. Then, the linear combination of the vectors defines the state (1.6), $\mathrm{P}[\psi]$, which is called linear superposition of the vector states $\mathrm{P}\left[\phi_{k}\right], k=1, \cdots, N$. This leads to interference phenomena that are purely quantum and unknown in the Newtonian mechanics such as the electron interference in the Tonomura [67] experiment (see Sec. 1.2.2).

The dynamical properties of quantum objects form a Boolean lattice similarly to the structural properties. This is to be understood in the following sense (cf.

\footnotetext{
${ }^{1}$ In Ref. [29], they were called 'conditional'.
} 
Ref. [48]). There is a set $\mathbf{M}$ of entities and a class $\mathbf{N}$ of possible properties of the entities. All entities having a given property form a subset that can be identified with the property. The family of all subsets form a Boolean lattice with the order relation, disjunction, conjunction and complement being the set-theoretical inclusion, intersection, union and complement in $\mathbf{M}$. This is analogous to the properties of a system in Newtonian mechanics: All systems of a given type form M. Each element of $\mathbf{M}$ is objectively in one point of the phase space of the system. Then, properties can be identified with subsets of the phase space.

The set of entities $\mathbf{M}$ that are considered in the present case consists of all quantum objects of the same type $\tau$. (Thus, they all have the same structural properties.) All states of any such object $\mathcal{S}$ that can be prepared form set $\mathbf{N}=\mathbf{T}\left(\mathbf{H}_{\tau}\right)_{1}^{+}$. Any $\mathbf{A} \subset \mathbf{T}\left(\mathbf{H}_{\tau}\right)_{1}^{+}$represents all copies of $\mathcal{S}$ that are prepared in the states contained in $\mathrm{A}^{2}$. Another example of $\mathbf{N}$ is the set of all gemenge structures (1.8) of a given state.

There is no finer or more complete description of a quantum system than by its gemenge structure. This implies that a prepared quantum system can be considered as a physical object in our theory 3 .

Some of the above statements seem to contradict the well-known fact that the subset of states consisting of all possible projections (quantum logic) does not form a Boolean lattice. In Sec. 3.2.2, the lattice structure of the set of projections will be described. It is clear that there is no contradiction because the lattice operations that are used in quantum logic are different from those introduced above. The deep reason is that the properties of quantum logic are associated with registrations and are, therefore, not objective, while our properties are determined by preparations and can be considered as objective.

\subsection{Observables}

Once an object in a state has been prepared, one can carry out a registration on it. Mathematically, a registration is described by an observable. A better understanding of the notion of state can be achieved if results of all possible registrations applied to the state can be calculated.

\footnotetext{
${ }^{2}$ Observe that the Boolean lattice of subsets of $\mathbf{N}$ has nothing to do with the Boolean lattice of preparation procedures as defined in Ref. [48]: the lattice operations are defined in a different way.

${ }^{3}$ In Ref. [48, p. 60, the problem is discussed whether a quantum system can be considered as a physical object. The criterion is that each state of the system is uniquely determined by its objective properties. Of course, this depends on what is a property. In Ref. [48, only values of observables are admitted as properties. Then, quantum systems are not physical objects. In our interpretation, the class of properties is much broader and certain quantum systems are, then, physical objects.
} 


\subsubsection{Mathematical preliminaries}

Definition 4 Let $\mathbf{F}$ be the Boolean lattice of all Borel subsets of $\mathbb{R}^{n}$. A positive operator valued (POV) measure

$$
\mathrm{E}: \mathbf{F} \mapsto \mathbf{L}_{r}(\mathbf{H})
$$

is defined by the properties

1. positivity: $\mathrm{E}(X) \geq 0$ for all $X \in \mathbf{F}$,

2. $\sigma$-additivity: if $\left\{X_{k}\right\}$ is a countable collection of disjoint sets in $\mathbf{F}$ then

$$
\mathrm{E}\left(\cup_{k} X_{k}\right)=\sum_{k} \mathrm{E}\left(X_{k}\right)
$$

where the series converges in weak operator topology, i.e., averages in any state converge to an average in the state.

3. normalisation:

$$
\mathrm{E}\left(\mathbb{R}^{n}\right)=1
$$

The number $n$ is called dimension of $\mathrm{E}$. Let us denote the support of the measure $\mathrm{E}$ by $\boldsymbol{\Omega}$. The set $\boldsymbol{\Omega}$ is called the value space of $\mathrm{E}$. The operators $\mathrm{E}(X)$ for $X \in \mathbf{F}$ are called effects.

The support $\Omega$ of measure $E$ is defined as follows

$$
\Omega=\left\{\vec{x} \in \mathbb{R}^{n} \mid \mathrm{E}(\{\vec{y}|| \vec{x}-\vec{y} \mid<\epsilon\}) \neq 0 \quad \forall \epsilon>0\right\} .
$$

Let $\mathbf{H}$ and $\mathbf{H}^{\prime}$ be two separable Hilbert spaces and $\mathbf{U}: \mathbf{H} \mapsto \mathbf{H}^{\prime}$ a unitary map. Then $\mathrm{UEU}^{\dagger}: \mathbf{F} \mapsto \mathbf{L}\left(\mathbf{H}^{\prime}\right)$ is a POV measure on $\mathbf{H}^{\prime}$.

We denote by $\mathbf{L}_{r}(\mathbf{H})_{\leq 1}^{+}$the set of all effects.

Proposition $4 \mathbf{L}_{r}(\mathbf{H})_{\leq 1}^{+}$is the set of elements of $\mathbf{L}_{r}(\mathbf{H})$ satisfying the inequality

$$
0 \leq \mathrm{E}(X) \leq 1
$$

That effects must satisfy (1.9) follows from the positivity and normalisation of a POV measure. On the other hand, each element of the set defined in Proposition 4 is an effect. For example, if $E$ is such an element, we can define $E(\{1\})=E$ and $E(\{-1\})=1-E$. The two operators satisfy Eq. (1.9) and they sum to 1, so they determine a POV measure with the value set $\Omega=\{-1,+1\}$.

Proposition 4 implies that the spectrum of each effect is a subset of $[0,1]$. An effect is a projection operator $\left(\mathrm{E}(X)^{2}=\mathrm{E}(X)\right)$ if an only if its spectrum is the two-point set $\{0,1\}$. 
Proposition 5 For any POV measure $\mathrm{E}: \mathbf{F} \mapsto \mathbf{L}(\mathbf{H})$ the following two conditions are equivalent

$$
\mathrm{E}(X)^{2}=\mathrm{E}(X)
$$

for all $X \in \mathbf{F}$ and

$$
\mathrm{E}(X \cap Y)=\mathrm{E}(X) \mathrm{E}(Y)
$$

for all $X, Y \in \mathbf{F}$.

Thus, a POV measure is a projection valued (PV) measure exactly when it is multiplicative. In this case, all effects commute with each other.

PV measures for $n=1$ are equivalent to s.a. operators that need not be bounded. Sec. 1.1.1 introduced only bounded s.a. operators and we need a more general definition.

Let $\mathbf{A}$ be an operator on Hilbert space $\mathbf{H}$ that is not necessarily bounded. Then it is not defined on the whole of $\mathbf{H}$ but only on linear subspace $\mathbf{D}_{\mathrm{A}}$ that is dense in $\mathbf{H}$ and is called domain of $\mathrm{A}$. The definition of adjoint has two steps: first, $\mathrm{A}^{\dagger}$ is defined on $\mathbf{D}_{\mathrm{A}}$ and then extended. Similarly the definition of the self-adjoint operator, see e.g. Ref. [63].

To define a sum and product of two unbounded operators A and B, their domains are used as follows. If

$$
\mathrm{D}_{\mathrm{A}}=\mathrm{D}_{\mathrm{B}}
$$

then we say that $A$ and $B$ have a common domain. If, moreover, the common domain $\mathbf{D}$ is invariant with respect to both operators, i.e.,

$$
\mathrm{AD} \subset \mathbf{D}, \quad \mathrm{BD} \subset \mathbf{D}
$$

then the sum $A+B$ and product $A B$ are well defined on $\mathbf{D}$ and can be possibly extended.

For s.a. operators, bounded or unbounded, the so-called spectral theorem holds, see Ref. 63]. This says that any s.a. operator A is equivalent to a PV measure, which is called, in this case, the spectral measure of $A$. Let $E$ be a PV measure, then $\mathrm{E}$ determines a unique self-adjoint operator $\int_{\mathbb{R}} \iota d \mathrm{E}$, where $\iota$ denotes the identity function on $\mathbb{R}$. Conversely, each s.a. operator $A$ on $\mathbf{H}$ determines a unique $\mathrm{PV}$ measure $\mathbf{E}: \mathbf{F} \mapsto \mathbf{L}(\mathbf{H})$ such that

$$
\mathrm{A}=\int_{\mathbb{R}} \iota d \mathrm{E} .
$$

If $P V$ measure $E$ is equivalent to a s.a. operator $A$, we shall denote it $E^{A}$. Thus, POV measure is a generalisation of a self-adjoint operator.

Another important special case is a discrete POV measure. A POV measure $\mathrm{E}$ is discrete if its value set $\Omega$ is an at most countable subset of $\mathbb{R}$. Let $\mathrm{E}$ be a discrete 
POV measure and let $\Omega=\left\{a_{k}, k \in \mathbb{N}\right\}$, where $\mathbb{N}$ is the set of positive integers. Then $\mathrm{E}$ is a $\mathrm{PV}$ measure, $\mathrm{E}=\mathrm{E}^{\mathrm{A}}, \mathrm{A}=\sum_{k} a_{k} \mathrm{E}_{k}, a_{k}$ are eigenvalues of $\mathrm{A}$ and

$$
\mathrm{E}_{k}=\mathrm{E}\left(\left\{a_{k}\right\}\right)
$$

are projections on the corresponding eigenspaces of the s.a. operator $A$ that is defined in this way.

For any POV measure $\mathbf{E}: \mathbf{F} \mapsto \mathbf{L}(\mathbf{H})$ and any $\mathrm{T} \in \mathbf{T}(\mathbf{H})_{1}^{+}$, the mapping

$$
p_{\mathrm{T}}^{\mathrm{E}}: \mathbf{F} \mapsto[0,1]
$$

defined by

$$
p_{\mathrm{\top}}^{\mathrm{E}}(X)=\operatorname{tr}[\mathrm{TE}(X)]
$$

for all $X \in \mathbf{F}$ is a real $\sigma$-additive probability measure with values in $[0,1]$. This follows from the defining properties of $E$ and the continuity and linearity of the trace. The measure is preserved by unitary maps,

$$
\operatorname{tr}\left[\left(\mathrm{UTU}^{\dagger}\right)\left(\mathrm{UE}(X) \mathrm{U}^{\dagger}\right)\right]=\operatorname{tr}[\mathrm{TE}(X)] .
$$

We note that a convex combination of states induces a convex combination of measures,

$$
\mathrm{\top}=\sum_{k} w_{k} \mathrm{\top}_{k} \mapsto p_{\mathrm{\top}}^{\mathrm{E}}=\sum_{k} w_{k} p_{\mathrm{\top}_{k}}^{\mathrm{E}}
$$

More about mathematical properties of states and effects and the corresponding spaces $\mathbf{T}(\mathbf{H})_{1}^{+}$and $\mathbf{L}_{r}(\mathbf{H})_{\leq 1}^{+}$can be found in Ref. [48].

\subsubsection{General rules}

In this subsection, we shall again work only with systems of definite type $\tau$.

Rule 2 Any quantum mechanical observable for system $\mathcal{S}$ of type $\tau$ is mathematically described by some POV measure $\mathbf{E}: \mathbf{F} \mapsto \mathbf{L}_{r}\left(\mathbf{H}_{\tau}\right)$. Each outcome of an individual registration of the observable $\mathrm{E}$ performed on an object $\mathcal{S}$ yields an element of $\mathbf{F}$. Each registration apparatus determines a unique observable.

Often, a stronger assumption is made, namely that each element of $\mathbf{L}_{r}\left(\mathbf{H}_{\tau}\right)$ is a sharp observable of system $\mathcal{S}$ of type $\tau$. Such systems are called proper quantum systems. In Secs. 2.2.2 and 6.2, we shall show that, in strict sense, no quantum system is proper. Usually, the construction of a model needs only few observables and, for a given model, our theory of quantum measurement will itself determine which POV measures cannot be observables.

An important assumption of quantum mechanics is the following generalisation of Born's rule 
Rule 3 The number $p_{\top}^{\mathrm{E}}(X)$ defined by $E q$. (1.11) is the probability that a registration of the observable $\mathrm{E}$ performed on object $\mathcal{S}$ in the state $\mathrm{T}$ leads to a result in the set $X$.

Using the same preparation $N$ times gives always the same state T. Performing in each case the registration measuring $E$ results in a value (or in an approximate value) $y \in \Omega$. The relative frequency of finding $y \in X$ approaches $p_{\boldsymbol{\top}}^{\mathrm{E}}(X)$ if $N \rightarrow \infty$. Hence, the observable is associated with an apparatus and the corresponding effects with subsets of values that can be obtained by registration processes.

Proposition 6 If $\mathrm{T}_{1}$ and $\mathrm{T}_{2}$ from $\mathbf{T}\left(\mathbf{H}_{\tau}\right)_{1}^{+}$satisfy

$$
\operatorname{tr}\left[\mathrm{T}_{1} \mathrm{E}\right]=\operatorname{tr}\left[\mathrm{T}_{2} \mathrm{E}\right]
$$

for all $\mathrm{E} \in \mathbf{L}_{r}\left(\mathbf{H}_{\tau}\right)_{\leq 1}^{+}$then $\mathrm{T}_{1}=\mathrm{T}_{2}$; if $\mathrm{E}_{1}$ and $\mathrm{E}_{2}$ from $\mathbf{L}_{r}\left(\mathbf{H}_{\tau}\right)_{\leq 1}^{+}$satisfy

$$
\operatorname{tr}\left[\mathrm{TE}_{1}\right]=\operatorname{tr}\left[\mathrm{TE}_{2}\right]
$$

for all $\mathbf{T} \in \mathbf{T}\left(\mathbf{H}_{\tau}\right)_{1}^{+}$then $\mathrm{E}_{1}=\mathrm{E}_{2}$.

Thus, a state $T$ can be determined uniquely, if it is prepared many times and a sufficient number of different registrations can be performed on it. As $\operatorname{tr}[\mathrm{ET}]$ is independent of the gemenge structure of $\mathrm{T}$, the question of what is its gemenge structure cannot be decided in this way with one exception: vector states. A vector state is extremal so that it cannot be a non-trivial gemenge.

In Rule 3, the probability $p_{\boldsymbol{\top}}^{\mathrm{E}}(X)$ refers to measurements on an object and not to the object itself. In general, the property that value $x \in \Omega$ lies within subset $X \in \mathbf{F}$ is not an objective property of objects in the sense that it could be attributed to an object itself and that the measurement would just reveal it. Such assumption would lead to contradictions, see Sec. 3.2.2. The property can be called a relational in the sense that it does not concern the quantum object alone but only in interaction with a definite registration device 4 . It can also be called incidental, because it is not predictable, except in special cases, see Sec. 3.2.2.

Thus, there is an asymmetry between states and observables: states are objective properties but observables are not; all elements of $\mathbf{T}\left(\mathbf{H}_{\tau}\right)_{1}^{+}$can be prepared but not all POV measures are observables.

Special cases in which the outcome of a registration is predictable are described as follows. Let $\mathbf{E}: \mathbf{F} \mapsto \mathbf{L}_{r}\left(\mathbf{H}_{\tau}\right)$ and let $\mathbf{T} \in \mathbf{T}\left(\mathbf{H}_{\tau}\right)_{1}^{+}$be such that

$$
\operatorname{tr}[\mathrm{TE}(X)]=1
$$

\footnotetext{
${ }^{4}$ There also are relational properties that are objective. Consider a point particle in Newtonian mechanics. Its angular momentum with respect to the origin of coordinates is an objective property of the particle if the origin is uniquely determined. It is relational, because it depends on the choice of origin, not only on the particle.
} 
for some $X \in \mathbf{F}$. Then the probability that the registration of $\mathrm{E}$ on $\mathrm{T}$ will give a value in $X$ is 1 and we can say that the prepared object in the state $T$ possesses the property independently of any measurement.

Proposition 7 The condition $\operatorname{tr}[\mathrm{TE}(X)]=1$ is equivalent to

$$
\mathrm{E}(X) \mathrm{T}=\mathrm{T} .
$$

In general, we call state $\mathrm{T}$ satisfying

$$
\mathrm{E}(X) \mathrm{T}=a \mathrm{\top}
$$

with some real a eigenstate of $\mathrm{E}(X)$ to eigenvalue $a$. If $\mathrm{T}=\mathrm{P}[\psi]$, then $\psi$ is called eigenvector. Thus, $\mathrm{T}$ in the Proposition 7 is an eigenstate of $\mathrm{E}(X)$ to eigenvalue 1.

If a PV measure is an observable, we call the observable sharp. Let us consider a discrete observable $A$ (which is always sharp) with spectrum $\left\{a_{k}\right\}$ that is nondegenerate, $\mathrm{P}_{k}=\mathrm{P}\left[\psi_{k}\right]$ for all $k . \psi_{k}$ are eigenvectors of $\mathrm{A}$. If we prepare the state $\mathrm{P}\left[\psi_{k}\right]$ and then register $\mathrm{A}$, the result must be $a_{k}$ with probability 1 . Next suppose that we prepare a linear superposition $\mathrm{P}[\Psi]$,

$$
\Psi=\sum_{1}^{\infty} c_{k} \psi_{k},
$$

with $\sum\left|c_{k}\right|^{2}=1$. The probability $p_{k}$ that the registration of $\mathrm{A}$ will give the result $a_{k}$ is

$$
p_{k}=\operatorname{tr}\left[\mathrm{P}\left[\psi_{k}\right] \mathrm{P}[\Psi]\right]=\left|c_{k}\right|^{2} .
$$

Eq. (1.12) gives the physical meaning to the coefficients in the linear superposition and is called Born rule. Observe that the probability is only meaningful if it concerns really existing (but may-be unknown) outcomes, see Sec. 3.1. Thus, it can be only associated with the registration, not with the object in state $\mathrm{P}[\Psi]$. One can say that, before the registration, the object in this state possesses all values $a_{k}$ for which $c_{k} \neq 0$ simultaneously.

This can be seen experimentally by means of the interference phenomena: the average in state $\mathrm{P}[\Psi]$ of observable $\mathrm{B}$ that does not commute with $\mathrm{A}$ does depend on matrix elements of $\mathbf{B}$ between different states $\psi_{k}$ :

$$
\operatorname{tr}[\mathrm{BP}[\Psi]]=\sum_{k l} c_{k}^{*} c_{l}\left\langle\psi_{k}|\mathrm{~B}| \psi_{l}\right\rangle .
$$

Rule 3 implies useful formulae for averages and higher moments of $E$ that generalise the well-known formulae for sharp observables. For the average $\langle E\rangle$ of observable $\mathrm{E}$ with $n=1$ in the state $\mathrm{T}$, we have

$$
\langle\mathrm{E}\rangle=\int_{\mathbb{R}} \iota d p_{\top}^{\mathrm{E}} .
$$


Using Eq. (1.11), we obtain

$$
\langle\mathrm{E}\rangle=\int_{\mathbb{R}} \iota d(\operatorname{tr}[\mathrm{TE}])=\operatorname{tr}\left[\mathrm{T} \int_{\mathbb{R}} \iota d \mathrm{E}\right] .
$$

For a sharp observable $\mathrm{E}^{\mathrm{A}}$, Eq. (1.10) yields the usual relation

$$
\langle\mathrm{A}\rangle=\operatorname{tr}[\mathrm{TA}] \text {. }
$$

In the case of vector state, $\mathrm{T}=\mathrm{P}[\phi]$, we obtain $\langle\mathrm{A}\rangle=\langle\phi \mid \mathrm{A} \phi\rangle$. For higher moments, we have just to substitute suitable power of $\iota$ in the integral and the proof is analogous.

The values of an observable are not objective properties of an object but its states are. As averages and moments of an observable are uniquely determined by states, we have

Proposition 8 The average $\langle\mathrm{E}\rangle$ of any observable $\mathrm{E}$ in state $\mathrm{T}$ of object $\mathcal{S}$ is an objective property of $\mathcal{S}$ that has been prepared in state $\mathrm{T}$.

One may wonder how an average can be objective if the values from which the average is calculated are not. However, the value $\langle E\rangle$ of the average is uniquely determined by the preparation and $\langle E\rangle$ is fixed before any registration, whereas the outcomes of individual registrations are not. In fact, the values that are obtained by registrations must satisfy the condition that they sum to a given average.

\subsection{Galilean group}

In quantum mechanics, Galilean relativity principle holds in the following form:

Rule 4 The same experiments performed in two different Newtonian inertial frames have the same results, i.e. give the same probability distributions.

'The same experiment' means that all conditions of the first experiment have the same description with respect to the first inertial frame as those of the second experiment have with respect to the second frame. In the present section, we restrict ourselves to the proper Galilean group and work out some consequences of the principle. For example, the most important PV measures of quantum mechanics will be defined and the time evolution equation formulated. We just list the relevant facts; for motivation and technical detail, see, e.g., Ref. [56].

The group of transformations that leave the geometric structure of Newtonian spacetime invariant5 (see, e.g., Ref. [50], p. 296) is called Galilean group G. It

\footnotetext{
${ }^{5}$ We understand symmetry as transformation that leaves some well-defined structure invariant. For example, Galilean group contains all transformations that leave Newtonian spacetime geometry invariant and a symmetry of a quantum system leaves its Hamiltonian invariant.
} 
is also the group of transformations between inertial frames. Let $\left(x_{1}, x_{2}, x_{3}, t\right)$ be a Newtonian inertial frame so that $x_{1}, x_{2}, x_{3}$ are Cartesian coordinates. A general element $g(\lambda)$ of the component of unity of $\mathbf{G}$ (called proper Galilean group, $\mathbf{G}^{+}$) can be written in the form

$$
\vec{x}^{\top} \mapsto \mathrm{O} \vec{x}^{\top}+\vec{a}^{\top}+\vec{v}^{\top} t
$$

and

$$
t \mapsto t+\lambda_{10},
$$

where $\mathrm{O}$ is a proper orthogonal matrix determined by three parameters $\lambda_{1}, \lambda_{2}, \lambda_{3}, \vec{a}$ is a vector of space shift with components $\lambda_{4}, \lambda_{5}, \lambda_{6}, \vec{v}$ is a relative velocity of the frames with components $\lambda_{7}, \lambda_{8}, \lambda_{9}$ and $\lambda_{10}$ is a time shift. The relations are written in matrix notation so that, e.g., $\vec{x}^{\top}$ is a column matrix with components of vector $\vec{x}$.

The group product $g\left(\lambda^{3}\right)=g\left(\lambda^{2}\right) g\left(\lambda^{1}\right)$ is defined as the composition of the transformations $g\left(\lambda^{2}\right)$ and $g\left(\lambda^{1}\right)$ so that $g\left(\lambda^{1}\right)$ is performed first and $g\left(\lambda^{2}\right)$ second. Then,

$$
\begin{gathered}
\mathrm{O}_{3}=\mathrm{O}_{2} \mathrm{O}_{1}, \\
\vec{a}_{3}^{\top}=\vec{a}_{2}^{\top}+\mathrm{O}_{2} \vec{a}_{1}^{\top}+\vec{v}_{2}^{\top} \lambda_{10}^{1}, \\
\vec{v}_{3}^{\top}=\vec{v}_{2}^{\top}+\mathrm{O}_{2} \vec{v}_{1}^{\top},
\end{gathered}
$$

and

$$
\lambda_{10}^{3}=\lambda_{10}^{2}+\lambda_{10}^{1} .
$$

Given a quantum system $\mathcal{S}$ of type $\tau$, classical apparatuses that are supposed to prepare and register $\mathcal{S}$ have well-defined Galilean transformations. There are then some conventions on the corresponding action on states and effects. Often, $\mathcal{S}$ is subject to influences external to $\mathcal{S}$ such as classical external fields. These also possess non-trivial transformation laws with respect to Galilean transformations. We call system $\mathcal{S}$ isolated, if the approximation that it is alone in the universe is a good one. Thus, there are no external influences.

Let us consider a measurement that consists of a preparation and a registration of system $\mathcal{S}$ of type $\tau$ by apparatuses $\mathcal{A}_{p}$ and $\mathcal{A}_{r}$, respectively, and let there be some external fields $f$. Let $\mathcal{A}_{p}$ prepare object $\mathcal{S}$ in state $\mathrm{T}$ and let $\mathcal{A}_{r}$ register effect $\mathrm{E}(X)$. Next, we can transport both $\mathcal{A}_{p}$ and $\mathcal{A}_{r}$ by $g \in \mathbf{G}$ to $g\left(\mathcal{A}_{p}\right)$ and $g\left(\mathcal{A}_{r}\right)$. Let $\mathrm{T}_{g, f}$ be the state prepared by $g\left(\mathcal{A}_{p}\right)$ and $\mathrm{E}_{g, f}(X)$ the effect registered by $g\left(\mathcal{A}_{r}\right)$. If the external influences are also transformed by $g: f \mapsto g(f)$, we obtain state $\mathrm{T}_{g, g(f)}$ and effect $\mathrm{E}_{g, g(f)}(X)$. The experiment has then been completely transferred by $g$ and, therefore, Galilean relativity principle implies 
Proposition 9 State $\mathrm{T}_{g, g(f)}$ and effect $\mathrm{E}_{g, g(f)}(X)$ satisfy

$$
\operatorname{tr}[\mathrm{TE}(X)]=\operatorname{tr}\left[\mathrm{T}_{g, g(f)} \mathrm{E}_{g, g(f)}(X)\right]
$$

for all $g \in \mathbf{G}, \mathbf{T} \in \mathbf{T}\left(\mathbf{H}_{\tau}\right)_{1}^{+}$and $\mathbf{E}(X) \in \mathbf{L}_{r}\left(\mathbf{H}_{\tau}\right)_{\leq 1}^{+}$.

Let us compare the two effects $\mathrm{E}(X)$ and $\mathrm{E}_{g, g(f)}(X)$. In the case that Borel sets $X$ have a well-defined transformation with respect to $g, \gamma(g): \mathbf{F} \mapsto \mathbf{F}$ and that the two effects belong to the same observable, we assume that

$$
\mathrm{E}_{g, g(f)}(X)=\mathrm{E}(\gamma(g) X) .
$$

Let us next compare measurable properties of the two states $\mathbf{T}$ and $\mathbf{T}_{g, g(f)}$. Proposition 9 implies:

$$
\operatorname{tr}\left[\mathrm{T}_{g, g(f)} \mathrm{E}(X)\right]=\operatorname{tr}\left[\mathrm{TE}_{g^{-1}, g^{-1}(f)}(X)\right] .
$$

Hence, values that can be registered on the state $\mathbf{T}_{g, g(f)}$ are those on $\mathrm{T}$ transformed by $g^{-1}$.

At this stage, one can formulate assumptions about the dependence of $\mathrm{T}_{g, f}$ and $\mathbf{E}_{g, f}(X)$ on $f$ and about the way $\mathbf{G}$ acts on $\mathbf{T}\left(\mathbf{H}_{\tau}\right)_{1}^{+}$and $\mathbf{L}_{r}\left(\mathbf{H}_{\tau}\right)_{\leq 1}^{+}$(see, e.g. Ref. [48]). Then, important theorems about action of $\mathbf{G}$ on $\mathbf{H}_{\tau}$ can be proved. However, it is simpler and for our purposes sufficient to assume directly:

Rule 5 Let $\mathcal{S}$ be an isolated system ( $f=0$ ) of type $\tau$ within which no separation status changes happen (see Sec. 2.2.4) and let $\overline{\mathbf{G}}^{+}$denote the universal covering of proper Galilean group. Then, for each element $g \in \overline{\mathbf{G}}^{+}$there is a unique map $\mathbf{U}(g): \mathbf{H}_{\tau} \mapsto \mathbf{H}_{\tau}$ so that

1. $\mathrm{P}[\mathrm{U}(g) \psi]=\mathrm{P}[\psi]_{g^{-1}, 0}$ for all $\psi \in \mathbf{H}_{\tau}$,

2. $g \mapsto \mathrm{U}(g)$ is a unitary ray representation of $\overline{\mathbf{G}}^{+}$depending on $\tau$,

3. for all $\mathbf{T} \in \mathbf{T}\left(\mathbf{H}_{\tau}\right)_{1}^{+}, \mathbf{E}(X) \in \mathbf{L}_{r}\left(\mathbf{H}_{\tau}\right)_{\leq 1}^{+}$and $g \in \overline{\mathbf{G}}^{+}$, we have

$$
\begin{aligned}
\mathrm{T}_{g^{-1}, 0} & =\mathrm{U}(g) \operatorname{TU}(g)^{\dagger}, \\
\mathrm{E}_{g^{-1}, 0}(X) & =\mathrm{U}(g) \mathrm{E}(X) \mathrm{U}(g)^{\dagger} .
\end{aligned}
$$

The 'ray' representation means that $\mathrm{U}\left(g_{2} g_{1}\right)=\exp \left[i \omega\left(g_{1}, g_{2}\right)\right] \mathrm{U}\left(g_{2}\right) \mathrm{U}\left(g_{1}\right)$. The representation depends of $\tau$. For example, the representation is irreducible for particles. Irreducible representations are classified by three numbers, $\mu, s$ and $V$, with the meaning of mass, spin and constant potential, respectively (see Ref. [46], p. 221). Clearly, the probability is preserved,

$$
\operatorname{tr}\left[\left(\mathrm{U}(g) \mathrm{TU}(g)^{\dagger}\right)\left(\mathrm{U}(g) \mathrm{E}(X) \mathrm{U}(g)^{\dagger}\right)\right]=\operatorname{tr}[\mathrm{TE}(X)]
$$


and we also have

$$
\left.\mathrm{U}(g) \mathrm{E}(X) \mathrm{U}(g)^{\dagger}\right)=\mathrm{E}\left(\gamma(g)^{-1} X\right)
$$

(see Ref. 20] for generalisation of this relation).

Now, the question arises: what is the form of spacetime transformations for systems that are not isolated? There are two different answers, one for Euclidean and one for time-translation subgroup of $\mathbf{G}$. This is a part of the asymmetry between time and space in quantum mechanics.

\subsubsection{Euclidean group}

Let $\mathbf{G}_{E}$ be the Euclidean subgroup of $\mathbf{G}$. We have:

Rule 6 Let $\mathcal{S}$ be a system of type $\tau$ not necessarily isolated. Then the conclusion of Rule 5 is valid only for the universal covering of proper Euclidean group, $\overline{\mathbf{G}}_{E}^{+}$, if $f=0$ is replaced by arbitrary $f$ and 'unitary ray representation' is replaced by 'unitary representation'.

The specific form of the representation $\mathrm{U}(g)$ depends on the type of $\mathcal{S}$ and can be described in all detail only after model assumptions on $\mathcal{S}$ are made (see Chap. 4). However, some general relations can be written already now.

If we restrict ourselves to the subgroup of space translations then the corresponding element of $\mathbf{G}_{E}$ can be denoted by $g(\vec{a})$ and its representative on $\mathbf{H}_{\tau}$ by $\mathbf{U}(\vec{a})$. Similarly, each rotation can be described by unit vector $\vec{n}$ along its axis and angle $\theta$ of rotation in the counter-clockwise direction around the axis. Let the corresponding elements of the rotation subgroup be $\mathrm{O}(\vec{n}, \theta)$ with representative $\mathrm{U}(\vec{n}, \theta)$.

Given a one-parameter group of unitary operators $U(\lambda)$, then according to Stone's theorem, there exists a s.a. operator G satisfying

$$
\mathrm{U}(\lambda)=\exp (i \mathrm{G} \lambda)
$$

It called of generator of group $\mathrm{U}(\lambda)$ and can be calculated with the help of the formula

$$
i \mathrm{G}=\mathrm{U}(\lambda)^{\dagger} \frac{d \mathrm{U}(\lambda)}{d \lambda}
$$

If the parameter is not specified by any convention, it is defined only up to a real multiplier and so is the generator.

Let us define a one-parameter subgroup by $\mathrm{U}(\lambda \vec{a})$. Its generator can be written in the form $(1 / i \hbar)\left(a_{1} \mathrm{P}_{1}+a_{2} \mathrm{P}_{2}+a_{3} \mathrm{P}_{3}\right)$, where $\mathrm{P}_{k}$ are three s.a. operators. Similarly, the generator of the subgroup $\mathrm{U}(\vec{n}, \lambda)$ has the form $(1 / i \hbar)\left(n_{1} \mathrm{~J}_{1}+n_{2} \mathrm{~J}_{2}+n_{3} \mathrm{~J}_{3}\right)$, where $\mathrm{J}_{k}$ are s.a. operators. 
Definition 5 The three s.a. operators $\mathrm{P}_{k}$ are three components, with respect of the coordinates $x_{1}, x_{2}, x_{3}$, of the total momentum and similarly $\mathrm{J}_{k}$ are components of total angular momentum of $\mathcal{S}$.

The commutators of $\mathrm{P}_{k}$ and $\mathrm{J}_{k}$ are determined by the fact that they are generators of $\mathbf{G}_{E}$ and that $g \mapsto \mathbf{U}(g)$ is a group representation:

$$
\left[\mathrm{P}_{k}, \mathrm{P}_{l}\right]=0, \quad\left[\mathrm{~J}_{k}, \mathrm{~J}_{l}\right]=i \hbar \epsilon_{k l j} \mathrm{~J}_{j}, \quad\left[\mathrm{~J}_{k}, \mathrm{P}_{l}\right]=i \hbar \epsilon_{k l j} \mathrm{P}_{j} .
$$

Most quantum systems admit position as one of their observables. All massive particles and all systems composite of a fixed number of massive particles, their position being their centre of mass, do. Given an inertial frame $(\vec{x}, t)$, one can measure the corresponding coordinates of the quantum system. We assume:

Rule 7 There are three s.a. operators $\mathrm{Q}_{k}$ such that the average position of object $\mathcal{S}$ in state $\mathrm{T}$ is $\operatorname{tr}\left[\mathrm{TQ}_{k}\right]$ and

$$
\mathrm{U}(\vec{a}) \mathrm{Q}_{k} \mathrm{U}(\vec{a})^{\dagger}=\mathrm{Q}_{k}-a_{k} 1
$$

and

$$
\mathrm{U}(\vec{n}, \theta) \mathrm{Q}_{k} \mathrm{U}(\vec{n}, \theta)^{\dagger}=\sum_{l} O(\vec{n},-\theta)_{k l} \mathrm{Q}_{l} .
$$

Hence, the position operators are transformed by Euclidean group in agreement with Eq. (1.13) and the corresponding operators $\mathrm{U}(g)$ transform $\mathrm{Q}_{k}$ according to Eq. (1.18). From these transformation properties, the commutation relations of position $\mathrm{Q}_{k}$ with total momentum $P_{k}$ and total angular momentum $J^{k}$ can be derived,

$$
\left[\mathrm{Q}_{k}, \mathrm{P}_{l}\right]=i \hbar \delta_{k l} 1, \quad\left[\mathrm{~J}_{k}, \mathrm{Q}_{l}\right]=i \hbar \epsilon_{k l j} \mathrm{Q}_{j} .
$$

Commutation rules (1.19) and (1.20) determine the action of operators $\mathrm{Q}_{k}$ and $\mathbf{P}_{k}$ on $\mathbf{H}_{\tau}$ uniquely. Consider the set $\mathbf{D}$ of rapidly decreasing $C^{\infty}$ functions $\phi(\vec{x}, \xi)$, where $\xi$ represents a set of parameters dependent on the system type $\tau$. For example, for a composite system, it can represent the relative coordinates of the constituents with respect to the centre of mass $\vec{x}$ and for a particle with spin, it can represent the components of the spin in some direction, etc. The rapid decrease is required with respect to $\vec{x}$ and those parameters $\xi$ that have unbounded ranges of values. In fact, $\mathbf{D}$ or its generalisation to functions of more than one argument is a common invariant domains of all s.a. operators in quantum mechanics and the products of these operators can be defined with the help of $\mathbf{D}$.

Let $\mathbf{H}_{\tau}$ be the completion of $\mathbf{D}$ with respect to the inner product

$$
\langle\phi \mid \psi\rangle=\sum_{\xi} \int_{\mathbb{R}^{3}} d^{3} x \phi^{*}(\vec{x}, \xi) \psi(\vec{x}, \xi),
$$


where the sum over $\xi$ represents sums over all discrete parameters and integrals over all continuous ones. The elements of $\mathbf{H}_{\tau}$ are called wave functions. To describe the operators, it is sufficient to define the action of their components on $C^{\infty}$ functions $\phi(\vec{x}, \xi)$ because there is always only one s.a. extension. The result is

$$
\mathrm{Q}_{k} \phi(\vec{x}, \xi)=x_{k} \phi(\vec{x}, \xi)
$$

and

$$
\mathrm{P}_{k} \phi(\vec{x}, \xi)=-i \hbar \frac{\partial}{\partial x_{k}} \phi(\vec{x}, \xi) .
$$

For the total angular momentum, the result is more complicated and $\tau$-dependent. We postpone its description to Chap. 4.

The form of Hilbert space and the operator described above is called $Q$-representation. All operators on $\mathbf{H}_{\tau}$ in $Q$-representation can be written in the form of integral operators with kernels

$$
\mathrm{A}=A\left(\vec{x}, \xi ; \vec{x}^{\prime}, \xi^{\prime}\right)
$$

the kernel $A\left(\vec{x}, \xi ; \vec{x}^{\prime}, \xi^{\prime}\right)$ being a generalised function of its arguments acting on functions $\phi(\vec{x}, \xi)$ as follows

$$
(\mathrm{A} \phi)(\vec{x}, \xi)=\sum_{\xi^{\prime}} \int_{\mathbb{R}^{3}} d^{3} x^{\prime} A\left(\vec{x}, \xi ; \vec{x}^{\prime}, \xi^{\prime}\right) \phi\left(\vec{x}^{\prime}, \xi^{\prime}\right),
$$

where the right-hand side represents the action of the generalised function on a test function.

\subsubsection{Time translations}

The next set of transformations are time translations. In quantum mechanics, time is, unlike position, just a parameter. This is another part of the asymmetry between time and space. For general systems (not necessarily closed), we assume

Rule 8 Let $\mathcal{S}$ be a system of type $\tau$ that does not take part in any separation status change (see Sec. 2.2.4) and let the external fields $f$ be given. Then, time translation from $t_{1}$ to $t_{2}$ is represented by unitary operator $\mathrm{U}\left(f, t_{2}, t_{1}\right)$ on $\mathbf{H}_{\tau}$ satisfying

$$
\mathrm{U}\left(f, t_{3}, t_{1}\right)=\mathrm{U}\left(f, t_{3}, t_{2}\right) \mathrm{U}\left(f, t_{2}, t_{1}\right)
$$

and we have

$$
\mathrm{P}[\psi]_{g\left(t_{2}-t_{1}\right)^{-1}, f}=\mathrm{U}\left(f, t_{2}, t_{1}\right) \mathrm{P}[\psi] \mathrm{U}\left(f, t_{2}, t_{1}\right)^{\dagger},
$$

where $g\left(t_{2}-t_{1}\right)$ is the group element for $\lambda_{1}=\cdots=\lambda_{9}=0$ and $\lambda_{10}=t_{2}-t_{1}$. 
A time translation does not define a unique map on $\mathbf{T}\left(\mathbf{H}_{\tau}\right)_{1}^{+}$in this case. $\mathbf{U}\left(f, t_{2}, t_{1}\right)$ depends not only on $t_{2}-t_{1}$ but also on the position of the system with respect to the external fields (even if the fields are stationary). We shall let out the argument $f$ in $\mathrm{U}\left(f, t_{2}, t_{1}\right)$ in agreement with the current practice.

Definition 6 The operator $\mathrm{H}(t)$ defined by

$$
\mathrm{H}(t)=i \hbar \mathrm{U}\left(t, t_{0}\right)^{\dagger} \frac{d \mathrm{U}\left(t, t_{0}\right)}{d t},
$$

is the Hamiltonian of $\mathcal{S}$.

Operators $\mathrm{H}(t)$ for different $t$ do not commute in general.

The most important observable in non-relativistic quantum mechanics is energy. The corresponding operator for system $\mathcal{S}$ is its Hamiltonian. Any individually prepared quantum systems has a definite Hamiltonian with the form

$$
\mathrm{H}=\mathrm{H} \text { (operators, external fields), }
$$

where function $\mathrm{H}$ symbolises the construction of the operator from the other s.a. operators of $\mathcal{S}$. The Hamiltonian of system $\mathcal{S}$ is an objective property of $\mathcal{S}$. Also, the form of Hamiltonian is a model assumption of quantum mechanics. The choice of a Hamiltonian is usually the most important step in model construction.

Hamiltonian $\mathrm{H}$ of quantum system $\mathcal{S}$ determines the dynamics of $\mathcal{S}$. Dynamics has to do with the time aspect of preparation and registration. Any preparation procedure finishes at some time instant $t_{p}$ and any registration procedure starts at some time instant $t_{r}$. The times $t_{r}$ and $t_{p}$ can serve as defining the time aspects because the whole preparation or registration processes can themselves take some time. The dynamics enables to calculate how the probabilities depend on the times $t_{r}$ and $t_{p}$. The dependence can be obtained in two ways. We can make either state $\mathrm{T}(t)$ to a function of $t$ by shifting $\mathrm{T}(0)$ forwards or observable $\mathrm{E}(t)$ by shifting it backwards by $\mathrm{U}(t, 0)$. The probability $p_{\mathrm{T}}^{\mathrm{E}}(X, t)$ corresponding to the time $t$ is then

$$
p_{\mathrm{\top}}^{\mathrm{E}}(X, t)=\operatorname{tr}[\mathrm{T}(t) \mathrm{E}]=\operatorname{tr}[\mathrm{TE}(t)] .
$$

The first method is called Schrödinger picture, the second Heisenberg picture.

Rule 8 implies:

Proposition 10 Let quantum system $\mathcal{S}$ have a Hamiltonian $\mathrm{H}(t)$. Then, the dynamical evolution of $\mathcal{S}$ in Schrödinger picture obeys von Neumann-Liouville equation of motion

$$
i \hbar \frac{d \mathrm{~T}(t)}{d t}=[\mathrm{H}(t), \mathrm{T}(t)]
$$

and in Heisenberg picture Heisenberg equation of motion

$$
i \hbar \frac{d \mathrm{E}(X, t)}{d t}=[\mathrm{E}(X, t), \mathrm{H}(t)] .
$$


Proposition 11 Let $\mathrm{T}$ be a vector state $|\phi\rangle\langle\phi|$. Then

$$
\mathbf{T}(t)=|\phi(t)\rangle\langle\phi(t)|,
$$

where $\phi(t)$ obeys Schrödinger equation

$$
i \hbar \frac{d \phi(t)}{d t}=\mathrm{H}(t) \phi(t)
$$

and

$$
\phi(t)=\mathrm{U}(t, 0) \phi(0) .
$$

It follows that Liouville-von-Neumann equation for states also determines an evolution equation for vectors. In particular, vector states remain vector states in evolution. More generally, we assume

Rule 9 Let $\mathrm{T}$ be a gemenge (1.8) with components $\mathrm{T}_{k}$. Then, its time evolution is again a gemenge with components $\mathrm{T}_{k}(t)$

$$
\mathbf{T}(t)=\left(\sum_{k}\right) w_{k} \mathrm{~T}_{k}(t),
$$

where $\mathrm{T}_{k}(t)$ is given by (1.23) and $w_{k}$ are time independent, for each $k$.

Of course, Eq. (1.26) is easily obtained from Eq. (1.8) by multiplying both sides by $U(t, 0)$ from the left and by $U(t, 0)^{\dagger}$ from the right. The non-trivial assumption is that the state remains a gemenge of the described kind. Hence, if an object $\mathcal{S}$ is prepared in the gemenge state $\mathrm{T}$ described by Eq. (1.8) at time 0 and then evolved, then the state of each individual object $\mathcal{S}$ is objectively always one of the components $\mathrm{T}_{k}(t)$ at time $t$.

Let us now restrict ourselves to isolated systems. In this case, the Hamiltonian $\mathbf{H}(t)=\mathbf{H}$ is time independent s.a. operator, the unitary time translation is

$$
\mathrm{U}\left(t_{2}, t_{1}\right)=\exp \left[-(i / \hbar) \mathrm{H}\left(t_{2}-t_{1}\right)\right]
$$

and

$$
\mathrm{U}\left(t_{2}, t_{1}\right)=\mathrm{U}\left(\lambda_{10}\right),
$$

where $\mathbf{U}\left(\lambda_{10}\right)$ is the representative of the time translation in the unitary representation of the Galilean group on $\mathbf{H}_{\tau}$ and $\lambda_{10}=t_{2}-t_{1}$. Clearly, the Hamiltonian must then commute with the total momentum and angular momentum,

$$
\left[\mathrm{H}, \mathrm{P}_{k}\right]=0, \quad\left[\mathrm{H}, \mathrm{J}_{k}\right]=0 .
$$


Definition 7 Each unitary transformation $\mathbf{U}: \mathbf{H}_{\tau} \mapsto \mathbf{H}_{\tau}$ that leaves the Hamiltonian $\mathrm{H}$ of $\mathcal{S}$ invariant,

$$
\mathrm{UHU}^{\dagger}=\mathrm{H}
$$

is called a symmetry of system $\mathcal{S}$.

All symmetries of a system $\mathcal{S}$ form a unitary group which is an objective property of $\mathcal{S}$. The generators of its one-parameter subgroups are s.a. operators that commute with the Hamiltonian and, as sharp observables, yield probability distributions that are independent of time.

We have ignored the improper transformations such as space inversions and time reversal. More about them can be found in Ref. [73]. 


\section{Chapter 2}

\section{Composition of quantum systems}

This is a key chapter of this Survey. It emphasises the difference between composition of systems of the same and of different types. It lists the problems to which the standard rules about composition of identical systems lead and it shows a rigorous way of removing these difficulties.

\subsection{Composition of systems of different types}

Suppose that two objects of different types are prepared. The objects can be particles or composite systems. Then, one can consider this pair of objects as one quantum object. This is called composition of objects. First, we describe the mathematical apparatus.

\subsubsection{Tensor product of Hilbert spaces}

The tensor product $\mathbf{H}=\mathbf{H}_{1} \otimes \mathbf{H}_{2}$ of $\mathbf{H}_{1}$ and $\mathbf{H}_{2}$ is the Cauchy completion of the linear span of the set of products

$$
\phi \otimes \psi: \phi \in \mathbf{H}_{1}, \psi \in \mathbf{H}_{2}
$$

with respect to the inner product of $\mathbf{H}$, which is determined by

$$
\left\langle\phi \otimes \psi \mid \phi^{\prime} \otimes \psi^{\prime}\right\rangle=\left\langle\phi \mid \phi^{\prime}\right\rangle\left\langle\psi \mid \psi^{\prime}\right\rangle .
$$

The tensor product $\phi \otimes \psi$ is linear in both arguments. Moreover, if $\left\{\phi_{k}\right\}$ and $\left\{\psi_{k}\right\}$ are bases of $\mathbf{H}_{1}$ and $\mathbf{H}_{2}$, then $\left\{\phi_{k} \otimes \psi_{l}\right\}$ is a basis of $\mathbf{H}$. If the bases are orthonormal then any $\Psi \in \mathbf{H}$ can then be expressed as

$$
\Psi=\sum_{k l}\left\langle\phi_{k} \otimes \psi_{l} \mid \Psi\right\rangle \phi_{k} \otimes \psi_{l} .
$$


If $\mathrm{A} \in \mathbf{L}\left(\mathbf{H}_{1}\right)$ and $\mathrm{B} \in \mathbf{L}\left(\mathbf{H}_{2}\right)$, then their tensor product $\mathrm{A} \otimes \mathrm{B}$ on $\mathbf{H}_{1} \otimes \mathbf{H}_{2}$ is determined via the relation

$$
(\mathrm{A} \otimes \mathbf{B})(\phi \otimes \psi)=\mathbf{A} \phi \otimes \mathbf{B} \psi
$$

for all $\phi \in \mathbf{H}_{1}$ and $\psi \in \mathbf{H}_{2}$. It follows that

$$
\operatorname{tr}[\mathrm{A} \otimes \mathrm{B}]=\operatorname{tr}[\mathrm{A}] \operatorname{tr}[\mathrm{B}] .
$$

The tensor product $\mathrm{T}_{1} \otimes \mathrm{T}_{2}$ of $\mathbf{T}_{1} \in \mathbf{T}\left(\mathbf{H}_{1}\right)_{1}^{+}$and $\mathbf{T}_{2} \in \mathbf{T}\left(\mathbf{H}_{2}\right)_{1}^{+}$is determined in the same way and it is a trace-class operator with trace 1. However, $\mathbf{T}\left(\mathbf{H}_{1} \otimes \mathbf{H}_{2}\right)_{1}^{+}$ contains more than just tensor products of elements from $\mathbf{T}\left(\mathbf{H}_{1}\right)_{1}^{+}$and $\mathbf{T}\left(\mathbf{H}_{2}\right)_{1}^{+}$.

The partial trace over the Hilbert space $\mathbf{H}_{2}$, say, is the positive linear mapping

$$
\Pi_{2}: \mathbf{T}\left(\mathbf{H}_{1} \otimes \mathbf{H}_{2}\right)_{1}^{+} \mapsto \mathbf{T}\left(\mathbf{H}_{1}\right)_{1}^{+}
$$

defined via the relation

$$
\operatorname{tr}\left[\Pi_{2}(\mathrm{~W}) \mathrm{A}\right]=\operatorname{tr}\left[\mathrm{W}\left(\mathrm{A} \otimes 1_{2}\right)\right]
$$

for all $\mathrm{A} \in \mathbf{L}\left(\mathbf{H}_{1}\right), \mathbf{W} \in \mathbf{T}\left(\mathbf{H}_{1} \otimes \mathbf{H}_{2}\right)$ and $1_{2}$ is the identity operator on $\mathbf{H}_{2}$. If $\left\{\phi_{k}\right\} \subset \mathbf{H}_{1}$ and $\left\{\psi_{k}\right\} \subset \mathbf{H}_{2}$ are orthonormal bases, then $\Pi_{2}(\mathbf{W})$ can be written as

$$
\Pi_{2}(\mathrm{~W})=\sum_{i j k}\left\langle\phi_{i} \otimes \psi_{k} \mid \mathrm{W}\left(\phi_{j} \otimes \psi_{k}\right)\right\rangle\left|\phi_{i}\right\rangle\left\langle\phi_{j}\right| .
$$

Here $\left|\phi_{i}\right\rangle\left\langle\phi_{j}\right|$ is the bounded linear operator on $\mathbf{H}_{1}$ given by

$$
\left|\phi_{i}\right\rangle\left\langle\phi_{j}\right|(\phi)=\left\langle\phi_{j} \mid \phi\right\rangle \phi_{i}
$$

for all $\phi \in \mathbf{H}_{1}$. The partial trace over $\mathbf{H}_{1}$ is defined similarly.

If $\mathrm{W}=\mathrm{T}_{1} \otimes \mathrm{T}_{2}$, then $\mathrm{T}_{1}=\Pi_{2}(\mathrm{~W})$ and $\mathrm{T}_{2}=\Pi_{1}(\mathrm{~W})$ but, in general,

$$
W \neq \Pi_{2}(W) \otimes \Pi_{1}(W) .
$$

In particular, if $\mathrm{W}=\mathrm{P}[\Psi]$, then

$$
\mathrm{P}[\Psi]=\Pi_{2}(\mathrm{P}[\Psi]) \otimes \Pi_{1}(\mathrm{P}[\Psi])
$$

if and only if

$$
\Psi=\phi \otimes \psi
$$

for some $\phi \in \mathbf{H}_{1}$ and $\psi \in \mathbf{H}_{2}$. In that case also

$$
\Pi_{2}(\mathrm{P}[\Psi])=\mathrm{P}[\phi], \quad \Pi_{1}(\mathrm{P}[\Psi])=\mathrm{P}[\psi] .
$$


Thus, tensor products of extremal elements of the sets $\mathbf{T}\left(\mathbf{H}_{1}\right)_{1}^{+}$and $\mathbf{T}\left(\mathbf{H}_{2}\right)_{1}^{+}$do not exhaust the set of extremal elements of $\mathbf{T}\left(\mathbf{H}_{1} \otimes \mathbf{H}_{2}\right)_{1}^{+}$.

Tensor products of more Hilbert spaces and the corresponding notions and relations can be obtained using the above rules.

Tensor product is also an operation for POV measures. Let $\mathrm{E}_{1}: \mathbf{F}_{1} \mapsto \mathbf{L}_{r}\left(\mathbf{H}_{1}\right)$ with dimension $n_{1}$ and $\mathbf{E}_{2}: \mathbf{F}_{2} \mapsto \mathbf{L}_{r}\left(\mathbf{H}_{2}\right)$ with dimension $n_{2}$ be two POV measures on Hilbert spaces $\mathbf{H}_{1}$ and $\mathbf{H}_{2}$ with value sets $\boldsymbol{\Omega}_{1}$ and $\boldsymbol{\Omega}_{2}$. Then POV measures $\left(\mathrm{E}_{1} \otimes \mathrm{E}_{2}\right):\left(\mathbf{F}_{1} \times \mathbf{F}_{2}\right) \mapsto \mathbf{L}_{r}\left(\mathbf{H}_{1} \otimes \mathbf{H}_{2}\right)$ on the tensor product $\mathbf{H}_{1} \otimes \mathbf{H}_{2}$ has dimension $n_{1}+n_{2}$ and values set $\boldsymbol{\Omega}_{1} \times \boldsymbol{\Omega}_{2}$ and is defined by

$$
\left(\mathrm{E}_{1} \otimes \mathrm{E}_{2}\right)\left(X_{1} \times X_{2}\right)=\mathrm{E}_{1}\left(X_{1}\right) \otimes \mathrm{E}_{2}\left(X_{2}\right)
$$

for all $X_{1} \subset \mathbb{R}^{n_{1}}$ and $X_{2} \subset \mathbb{R}^{n_{2}}$. Tensor product of POV measures is associative but not commutative.

Let us now turn to physical interpretation.

Rule 10 Let $\mathcal{S}_{1}$ and $\mathcal{S}_{2}$ be two quantum objects of different types and their Hilbert spaces be $\mathbf{H}_{1}$ and $\mathbf{H}_{2}$, respectively. Then, the object $\mathcal{S}$ composed of $\mathcal{S}_{1}$ and $\mathcal{S}_{2}$ has the Hilbert space $\mathbf{H}=\mathbf{H}_{1} \otimes \mathbf{H}_{2}$, its states are elements of $\mathbf{T}\left(\mathbf{H}_{1} \otimes \mathbf{H}_{2}\right)_{1}^{+}$and its effects are elements of $\mathbf{L}_{r}\left(\mathbf{H}_{1} \otimes \mathbf{H}_{2}\right)_{\leq 1}^{+}$

Rule 11 Let $\mathrm{T}$ be a state of a composite object $\mathcal{S}+\mathcal{S}^{\prime}$. The necessary and sufficient condition for the gemenge structure of the partial trace over $\mathcal{S}^{\prime}$ to be

$$
\operatorname{tr}_{\mathcal{S}^{\prime}}[\mathrm{T}]=\left(\sum_{k}\right) w_{k} \mathrm{~T}_{k}
$$

is that $\mathrm{T}$ itself has the gemenge structure

$$
\mathrm{T}=\left(\sum_{k}\right) w_{k} \mathrm{~T}_{k} \otimes \mathrm{T}_{k}^{\prime},
$$

where $\mathrm{T}_{k}^{\prime}$ are some states of $\mathcal{S}^{\prime}$.

One can say that gemenge structures are invariant with respect to compositions. By registration of observables of $\mathcal{S}$ alone, only the state operator $\mathrm{T}$ defined by Eq. (1.8) can be determined. However, by registration of observables pertaining to a composite object $\mathcal{S}+\mathcal{S}^{\prime}$, some information about the gemenge structure of $\mathrm{T}$ can be obtained from Rule 11. Suppose, e.g., that $\mathcal{S}+\mathcal{S}^{\prime}$ is in a vector state $\mathrm{P}[\Psi]$. Then partial trace $\Pi^{\prime}(\mathrm{P}[\Psi])$ cannot be a gemenge. This fact is at the root of the objectification problem in quantum theory of measurement (cf. [16], our Secs. 6.1 and 6.3). 
If the state of the composite object $\mathcal{S}_{1}+\mathcal{S}_{2}$ is $\mathbf{W} \in \mathbf{T}\left(\mathbf{H}_{1} \otimes \mathbf{H}_{2}\right)_{1}^{+}$, then the two partial trace operations determine unique states, $\Pi_{2}(\mathrm{~W})$ of $\mathcal{S}_{1}$ and $\Pi_{1}(\mathrm{~W})$ of $\mathcal{S}_{2}$. These states are called reduced states. The fact that the reduced state $\Pi_{2}(\mathrm{~W})$ is uniquely determined by $\mathrm{W}$ means, in particular, that objects $\mathcal{S}_{1}$ and $\mathcal{S}_{2}$ can be identified as subsystems of $\mathcal{S}_{1}+\mathcal{S}_{2}$.

Based on the mathematical properties of the tensor products, the description of objects composed of arbitrary number of sub-systems can be obtained by extension of the above methods. The only condition is that no two subsystems belong to the same type.

\subsubsection{Entanglement}

Definition 8 If object $\mathcal{S}$ composite of two quantum objects $\mathcal{S}_{1}$ and $\mathcal{S}_{2}$ is in a state W that satisfies condition (2.1) one says that $\mathcal{S}_{1}$ and $\mathcal{S}_{2}$ are entangled or that state $\mathrm{W}$ is entangled.

Entanglement is a physical phenomenon that has measurable consequences. One of these consequences are correlations between outcomes of registrations that are performed jointly on the two (or more) entangled systems.

As an example, consider two objects $\mathcal{S}_{1}$ and $\mathcal{S}_{2}$ and two sharp observables $\mathrm{A}_{i}$ : $\mathbf{H}_{i} \mapsto \mathbf{H}_{i}, i=1,2$. Let $\left|a_{i}\right\rangle \in \mathbf{H}_{i}$ and $\left|b_{i}\right\rangle \in \mathbf{H}_{i}$ be four eigenstates,

$$
\mathrm{A}_{i}\left|a_{i}\right\rangle=a_{i}\left|a_{i}\right\rangle, \quad \mathrm{A}_{i}\left|b_{i}\right\rangle=b_{i}\left|b_{i}\right\rangle,
$$

and $b_{i}>a_{i}, i=1,2$. The state $\mathrm{P}[\Psi]$ of the composite object $\mathcal{S}_{1}+\mathcal{S}_{2}$, where

$$
|\Psi\rangle=\frac{1}{\sqrt{2}}\left(\left|a_{1}\right\rangle \otimes\left|b_{2}\right\rangle+\left|b_{1}\right\rangle \otimes\left|a_{2}\right\rangle\right)
$$

is entangled. Indeed,

$$
\Pi_{2}(\mathrm{P}[\Psi])=\frac{1}{2}\left(\left|a_{1}\right\rangle \otimes\left\langle a_{1}|+| b_{1}\right\rangle \otimes\left\langle b_{1}\right|\right), \quad \Pi_{1}(\mathrm{P}[\Psi])=\frac{1}{2}\left(\left|a_{2}\right\rangle \otimes\left\langle a_{2}|+| b_{2}\right\rangle \otimes\left\langle b_{2}\right|\right)
$$

and $\mathrm{P}[\Psi]$ is not a tensor product of these two states.

Let us calculate the correlations of two sharp observables, $A_{1} \otimes 1$ and $1 \otimes A_{2}$ on $\mathbf{H}_{1} \otimes \mathbf{H}_{2}$. The observables commute hence we can measure them jointly (see Sec. 3.2). First, we need:

Definition 9 Let $\mathrm{A}$ be a s.a. operator with a dense invariant domain and $\mathrm{T}$ be an arbitrary state. Then

$$
\Delta \mathrm{A}=\operatorname{tr}\left[\mathrm{T}\left(\sqrt{\mathrm{A}^{2}-\langle\mathrm{A}\rangle^{2}}\right)\right]
$$

is called variance of $\mathrm{A}$ in $\mathrm{T}$. 
Second,

Definition 10 Normalised correlation of any two observables $\mathrm{A}$ and $\mathrm{B}$ in a state $\mathrm{T}$ is defined by

$$
C(\mathrm{~A}, \mathrm{~B}, \mathrm{~T})=\frac{\operatorname{tr}[\mathrm{TAB}]-\operatorname{tr}[\mathrm{TA}] \operatorname{tr}[\mathrm{TB}]}{\Delta \mathrm{A} \Delta \mathrm{B}} .
$$

The normalised correlation satisfies

$$
-1 \leq C(\mathrm{~A}, \mathrm{~B}, \mathrm{~T}) \leq 1
$$

for all $A, B$ and $T$ because of Schwarz' inequality. The proof is based on the facts that $\operatorname{Re} \operatorname{tr}[\mathrm{TAB}]$ is a positive symmetric bilinear form on real linear space $\mathbf{L}_{r}\left(\mathbf{H}_{1} \otimes\right.$ $\left.\mathbf{H}_{2}\right)$, Retr $[\mathrm{TAB}]=\operatorname{tr}[\mathrm{TAB}]$ if $\mathrm{A}$ and $\mathrm{B}$ commute and their correlation can only be measured if they commute. If $C(\mathrm{~A}, \mathrm{~B}, \mathrm{~T})=1$, the operators are strongly correlated, if $C(\mathrm{~A}, \mathrm{~B}, \mathrm{~T})=0$, they are uncorrelated and if $C(\mathrm{~A}, \mathrm{~B}, \mathrm{~T})=-1$, they are strongly anticorrelated (see [16], p. 50).

First, we calculate their first two moments of observables $A_{1} \otimes 1$ and $1 \otimes A_{2}$ in state $\mathrm{P}[\Psi]$ :

$$
\left\langle\Psi\left|\mathrm{A}_{1} \otimes 1\right| \Psi\right\rangle=\frac{1}{2}\left(a_{1}+b_{1}\right), \quad\left\langle\Psi\left|1 \otimes \mathrm{A}_{2}\right| \Psi\right\rangle=\frac{1}{2}\left(a_{2}+b_{2}\right)
$$

and

$$
\left\langle\Psi\left|\left(\mathrm{A}_{1} \otimes 1\right)^{2}\right| \Psi\right\rangle=\frac{1}{2}\left(a_{1}^{2}+b_{1}^{2}\right), \quad\left\langle\Psi\left|\left(1 \otimes \mathrm{A}_{2}\right)^{2}\right| \Psi\right\rangle=\frac{1}{2}\left(a_{2}^{2}+b_{2}^{2}\right) .
$$

The variances are

$$
\Delta\left(\mathrm{A}_{1} \otimes 1\right)=\left(\frac{b_{1}-a_{1}}{2}\right)^{2}, \quad \Delta\left(1 \otimes \mathrm{A}_{2}\right)=\left(\frac{b_{2}-a_{2}}{2}\right)^{2} .
$$

The average of the product $\left(A_{1} \otimes 1\right)\left(1 \otimes A_{2}\right)=\left(A_{1} \otimes A_{2}\right)$ is

$$
\left\langle\Phi \mid \mathrm{A}_{1} \otimes \mathrm{A}_{2} \Phi\right\rangle=\frac{a_{1} b_{2}+b_{1} a_{2}}{2}
$$

so that, finally

$$
C\left(\mathrm{~A}_{1} \otimes 1,1 \otimes \mathrm{A}_{2}, \mathrm{P}[\Psi]\right)=-1
$$

The result is that the observables are strongly anticorrelated in state $\mathrm{P}[\Psi]$. What this means, can be seen from the probability distributions for different possible outcomes by measuring the two observables. The corresponding PV measures define the four projections

$$
\begin{array}{ll}
\mathrm{P}_{a a}=\mathrm{E}_{1}\left(\left\{a_{1}\right\}\right) \otimes \mathrm{E}_{2}\left(\left\{a_{2}\right\}\right), & \mathrm{P}_{a b}=\mathrm{E}_{1}\left(\left\{a_{1}\right\}\right) \otimes \mathrm{E}_{2}\left(\left\{b_{2}\right\}\right), \\
\mathrm{P}_{b a}=\mathrm{E}_{1}\left(\left\{b_{1}\right\}\right) \otimes \mathrm{E}_{2}\left(\left\{a_{2}\right\}\right), & \mathrm{P}_{b b}=\mathrm{E}_{1}\left(\left\{b_{1}\right\}\right) \otimes \mathrm{E}_{2}\left(\left\{b_{2}\right\}\right),
\end{array}
$$


and we obtain

$$
\begin{aligned}
& \left\langle\Psi\left|\mathrm{P}_{a a}\right| \Psi\right\rangle=0, \quad\left\langle\Psi\left|\mathrm{P}_{a b}\right| \Psi\right\rangle=\frac{1}{2}, \\
& \left\langle\Psi\left|\mathrm{P}_{b a}\right| \Psi\right\rangle=\frac{1}{2}, \quad\left\langle\Psi\left|\mathrm{P}_{b b}\right| \Psi\right\rangle=0 .
\end{aligned}
$$

It follows: if the registration of $\mathrm{A}_{1} \otimes 1$ gives $a_{1}\left(b_{1}\right)$ then the registration of $1 \otimes \mathrm{A}_{1}$ gives $b_{2}\left(a_{2}\right)$ with certainty, and vice versa, the correlation being symmetric with respect to $\mathcal{S}_{1}$ and $\mathcal{S}_{2}$.

Suppose now that $\mathcal{S}_{1}$ and $\mathcal{S}_{2}$ are far from each other, $\mathcal{S}_{1}$ near point $\vec{x}_{1}$ and $\mathcal{S}_{2}$ near point $\vec{x}_{2}$. Could one use the strong anticorrelation to send signals from $\vec{x}_{1}$ to $\vec{x}_{2}$, say? There are two reasons why one cannot. First, one has no choice of what value, $a_{1}$ or $b_{1}$ one obtains at $\vec{x}_{1}$. As is easily seen from the state $\Pi_{2}(\mathrm{P}[\Psi])$ of $\mathcal{S}_{1}$, Eq. (2.2), the probability of each outcome is $1 / 2$. One has, therefore, no control about what signal will be sent. Second, suppose that the state $\mathrm{P}[\Psi]$ is prepared many times and let, in the first chain of experiments, the observer at $\vec{x}_{1}$ registers $A_{1}$ every time, in the second chain, he does nothing. Is there any difference between the two cases that could be recognized at $\vec{x}_{2}$ ? In the first case, the state of $\mathcal{S}_{2}$ is given by Eq. (2.2) including its gemenge structure, whereas, in the second case, the state operator is the same but it is not a gemenge because the composite system is in a vector state. However, by registration of any observable pertinent to $\mathcal{S}_{2}$, the observer at $\vec{x}_{2}$ cannot distinguish different gemenge structures of the same state operator from each other.

The next interesting question is, how the influence of a registered value at $\vec{x}_{1}$ on that at $\vec{x}_{2}$ is to be understood. Even in classical mechanics, one can arrange strong correlations. For example, if a body with zero angular momentum with respect to its centre of mass decays into two bodies flying away from each other, the angular momentum of the first is exactly the opposite to that of the second. This strong anticorrelation cannot be used to send signals, either. It is moreover clear, that a measurement of the first angular momentum giving the value $\vec{L}_{1}$ is not a cause of the second angular momentum having the value $-\vec{L}_{1}$. Rather, the decay is the common cause of the two values being opposite. The process of creating the two opposite values at distant points is also completely local: the decay is a local phenomenon and the movement of each of the debris is governed by a local equation of motion. And, the values $\vec{L}_{1}$ and $-\vec{L}_{1}$ are objective, that is, they exist on the debris independently of any measurement and can be, in this way, transported from the decay point to the measurements in a local way.

In quantum mechanics, such an explanation of the correlations is not possible. A value of an observable is created only during its registration. It does not exist in any form before the registration, except in the special case of state which is an eigenstate. However, in our example, $\Psi$ is not an eigenstate either of $A_{1} \otimes 1$ or of $1 \otimes \mathrm{A}_{2}$. The registrations performed simultaneously at $\vec{x}_{1}$ and at $\vec{x}_{2}$, which can be 
very far from each other, are connected by a relation that is utterly non-local. How can the apparatuses together with parts of the quantum system at two distant points $\vec{x}_{1}$ and at $\vec{x}_{2}$ 'know' what values they are to create in order that the correlations result? Note that the rejection of objectivity of observables leads more directly to non-locality than assumption of some sort of realism.

The nature of simultaneity with which the correlations take place can be described in more details, see Ref. [37].

The non-local correlation between the registration outcomes can be ascertained only if both values are known and can be compared. It may therefore be more precise if we say that the non-local correlations can only be seen if the non-local observable $A_{1} \otimes A_{2}$ is registered. This non-locality of quantum correlations in entangled states does not lead to any internal contradictions in quantum mechanics and is compatible with other successful theories (such as special relativity) as well as with existing experimental data. Nonetheless it is very surprising and it has been very thoroughly studied. In this way, various conditions (e.g., Bell inequality) have been found that had to be satisfied by values of observables if they were objective and locality were satisfied. Experiments show that such conditions are violated, and, moreover, that their violation can even be exploited in quantum communication techniques [14].

Finally, let us repeat here that non-objectivity of observables is accepted by our interpretation of quantum mechanics in the full extent and that this does not lead to any contradiction with Basic Ontological Hypothesis of Quantum Mechanics and with our realist interpretation.

\subsection{Composition of identical systems}

If a prepared object has more than one subsystem of the same type (identical subsystems), then these subsystems are indistinguishable. This idea can be mathematically expressed as invariance with respect to permutations.

\subsubsection{Identical subsystems}

Let $\mathbf{S}_{N}$ be the permutation group of $N$ objects, that is, each element $g$ of $\mathbf{S}_{N}$ is a bijective map $g:\{1, \cdots, N\} \mapsto\{1, \cdots, N\}$, the inverse element to $g$ is the inverse map $g^{-1}$ and the group product of $g_{1}$ and $g_{2}$ is defined by $\left(g_{1} g_{2}\right)(k)=g_{1}\left(g_{2}(k)\right)$, $k \in\{1, \cdots, N\}$.

Given a Hilbert space $\mathbf{H}$, let us denote by $\mathbf{H}^{N}$ the tensor product of $N$ copies of H,

$$
\mathbf{H}^{N}=\mathbf{H} \otimes \mathbf{H} \otimes \cdots \otimes \mathbf{H} .
$$


On $\mathbf{H}^{N}$, the permutation group $\mathbf{S}_{N}$ acts as follows. Let $\psi_{k} \in \mathbf{H}, k=1, \cdots, N$, then

$$
\psi_{1} \otimes \cdots \otimes \psi_{N} \in \mathbf{H}^{N}
$$

and

$$
\mathrm{g}\left(\psi_{1} \otimes \cdots \otimes \psi_{N}\right)=\psi_{g(1)} \otimes \cdots \otimes \psi_{g(N)}
$$

$\mathrm{g}$ preserves the inner product of $\mathbf{H}^{N}$ and is, therefore, bounded and continuous. Hence, it can be extended by linearity and continuity to the whole of $\mathbf{H}^{N}$. The resulting operator on $\mathbf{H}^{N}$ is denoted by the same symbol $\mathrm{g}$ and is a unitary operator by construction. The action (2.5) thus defines a unitary representation of the group $\mathbf{S}_{N}$ on $\mathbf{H}^{N}$.

All vectors of $\mathbf{H}^{N}$ that transform according to a fixed unitary representation $\mathcal{R}$ of $\mathbf{S}_{N}$ form a closed linear subspace of $\mathbf{H}^{N}$ that will be denoted by $\mathbf{H}_{\mathcal{R}}^{N}$. The representations being unitary, the subspaces $\mathbf{H}_{\mathcal{R}}^{N}$ are orthogonal to each other. Let us denote by $\mathrm{P}_{\mathcal{R}}^{(N)}$ the orthogonal projection operator,

$$
\mathrm{P}_{\mathcal{R}}^{(N)}: \mathbf{H}^{N} \mapsto \mathbf{H}_{\mathcal{R}}^{N}
$$

The location order of a given state in a tensor product can be considered as an information about the identity of the corresponding system. Such information has no physical meaning and a change of the ordering is just a kind of gauge transformation 1 . Motivated by this idea, we look for one-dimensional unitary representations of $\mathbf{S}_{N}$ because only these transform vectors by a phase factor multiplication. $\mathbf{S}_{N}$ has exactly two one-dimensional unitary representations: the symmetric (trivial) one, $g \mapsto \mathbf{1}$, and the alternating one $g \mapsto \eta(g) \mathbf{1}$ for each $g \in \mathbf{S}_{N}$, where $\eta(g)=1$ for even permutations $g$ and $\eta(g)=-1$ for odd permutations $g$. If $\mathcal{R}$ is the symmetric (alternating) representation we use symbol $\mathbf{H}_{s}^{N}\left(\mathbf{H}_{a}^{N}\right)$ for $\mathbf{H}_{\mathcal{R}}^{N}$. Let $\mathrm{P}_{s}^{(N)}\left(\mathrm{P}_{a}^{(N)}\right)$ be the orthogonal projection on $\mathbf{H}_{s}^{N}\left(\mathbf{H}_{a}^{N}\right)$. Note that the usual operation of symmetrisation or antisymmetrisation on a vector $\Psi \in \mathbf{H}^{N}$, such as

$$
\psi \otimes \phi \mapsto(1 / 2)(\psi \otimes \phi \pm \phi \otimes \psi)
$$

in $\mathbf{H}^{2}$, is nothing but $\mathrm{P}_{s}^{(N)} \Psi$ or $\mathrm{P}_{a}^{(N)} \Psi$, respectively.

Now, we are ready to formulate the basic assumption concerning identical subsystems. From relativistic quantum field theory [72], we take over the following result.

Rule 12 Let $\mathcal{S}^{N}$ be a composite quantum object consisting of $N$ subsystems $\mathcal{S}$, each of type $\tau$ with Hilbert space $\mathbf{H}_{\tau}$. Then, with important exceptions to be specified

\footnotetext{
${ }^{1} \mathrm{~A}$ different and independent part (ignored here) of the theory of identical particles is that states of two identical systems can also be swapped in a physical process of continuous evolution, and can so entail a non-trivial phase factor at the total state (anyons, see, e.g. Ref. [74]).
} 
later, the Hilbert space of $\mathcal{S}^{N}$ is $\mathbf{H}_{\tau s}^{N}$ for subsystems with integer spin and $\mathbf{H}_{\tau a}^{N}$ for those with half-integer spin.

Definition 11 Systems with integer spin are called bosons and those with halfinteger spin are called fermions. The symmetry properties of states leads to BoseEinstein statistics for bosons and Fermi-Dirac one for fermions.

We can, therefore, introduce a useful notation, a common symbol $\mathbf{H}_{\mathcal{R}(\tau)}^{N}$ for the subspaces of the symmetric and anti-symmetric representations and $\mathrm{P}_{\tau}^{(N)}$ for the corresponding projections because the representation is determined by the system type $\tau$.

Proposition 12 The states of any system $\mathcal{S}^{N}$ consisting of subsystems of type $\tau$ for which Rule 12 holds are elements of $\mathbf{T}\left(\mathbf{H}_{\mathcal{R}(\tau)}^{N}\right)_{1}^{+}$and the effects of $\mathcal{S}^{N}$ are elements of $\mathbf{L}_{r}\left(\mathbf{H}_{\mathcal{R}(\tau)}^{N}\right)_{\leq 1}^{+}$.

Proposition 12 follows directly from the definition of a system with a given Hilbert space. To see its significance, let us make a comparison with the case of subsystems of different types. First, one has to realise the obvious fact that observing anything on a subsystem is tantamount to observing something on the whole system. The application of this rule to system $\mathcal{S}^{\prime}$ composite of two subsystems $\mathcal{S}_{1}$ and $\mathcal{S}_{2}$ of different types has the following mathematical expression. Any effect $\mathrm{E}_{1}$ of $\mathcal{S}_{1}$ determines uniquely the effect $\mathrm{E}_{1} \otimes 1_{2}$ of $\mathcal{S}^{\prime}$. Thus, even if $\mathcal{S}_{1}$ is a part of $\mathcal{S}^{\prime}$, we can measure $\mathrm{E}_{1}$ on it. Similarly, if state $\mathrm{P}\left[\phi_{1}\right]$ of $\mathcal{S}_{1}$ and state $\mathrm{P}\left[\phi_{2}\right]$ of $\mathcal{S}_{2}$ are prepared independently, then the state of the composed system is $\mathrm{P}\left[\phi_{1}\right] \otimes \mathrm{P}\left[\phi_{2}\right]$ and the partial traces give back the originally prepared states of the subsystems. Thus, composing such systems does not disturb their individuality and rules valid for each of them separately.

Looking at the composition of identical systems we observe a very different picture. For instance, consider system $\mathcal{S}^{2}$ of two bosons of type $\tau$ with common Hilbert space $\mathbf{H}_{\tau}$. The Hilbert space of $\mathcal{S}^{2}$ is $\mathbf{H}_{\mathcal{R}(\tau)}^{2}$. Let $\left\{\psi_{k}\right\}$ be an orthonormal basis of $\mathbf{H}_{\tau}$. Then, the set of vectors

$$
\psi_{k} \otimes \psi_{k}, \quad \forall k
$$

and

$$
\frac{1}{\sqrt{2}}\left(\psi_{k} \otimes \psi_{l}+\psi_{l} \otimes \psi_{k}\right), \quad \forall k \neq l
$$

is an orthonormal basis for $\mathbf{H}_{\mathcal{R}(\tau)}^{2}$. Hence, if Preparation I prepares a boson in state $\mathrm{P}\left[\phi_{k}\right]$ and independent Preparation II prepares another boson in $\mathrm{P}\left[\phi_{l}\right], k \neq l$, then neither boson seems to be in its individually prepared state. Clearly, the notion of individual states as partial traces does not make sense for particles of the same type. Moreover, the notion of preparation itself becomes problematical. 
Next, let $\mathrm{A}$ be any operator on $\mathbf{H}_{\tau}$. Then, neither $\mathrm{A} \otimes 1$ nor $1 \otimes \mathrm{A}$ are operators on $\mathbf{H}_{\mathcal{R}(\tau)}^{2}$ because they map any element of the above basis out of $\mathbf{H}_{\mathcal{R}(\tau)}^{2}$. Thus, they are not observables of any of the bosons. As $\mathbf{H}_{\tau}$ is the Hilbert space of each boson constituent of $\mathcal{S}^{2}$, the effects of each boson would have to be operators on $\mathbf{H}_{\tau}$ according to Rule 2.

It seems therefore that unrestricted validity of Proposition 12 would contradict Rules of Secs. 1.1.2 and 1.2.2 on prepared states and form of registrable observables. Clearly all this can be generalised to any number of both boson and fermion constituents. Let us study this problem in more detail.

\subsubsection{Cluster separability}

The problem described in the previous section is aggravated if the two systems are prepared in different laboratories. It seems that experiments with one object might be disturbed by another object of the same type, even if it were prepared independently, far away from the first. One can avoid similar problems by adding some assumption of locality to the axioms of quantum theory.

The relativistic theory starts with the requirement that space-time symmetries of an isolated system (i.e., that is alone in space) be realised by unitary representations of Poincaré group on the Hilbert space of states, see Refs. [72] and [28]. Then, the cluster decomposition principle, a locality assumption, states that if multi-particle scattering experiments are studied in distant laboratories, then the $S$-matrix element for the overall process factorizes into those concerning only the experiments in the single laboratories. This ensures a factorisation of the corresponding transition probabilities, so that an experiment in one laboratory cannot influence the results obtained in another one. Cluster decomposition principle implies non-trivial local properties of the theory underlying the $S$-matrix, in particular it plays a crucial part in making local field theory inevitable (cf. Ref. [72], Chap. 4).

In the phenomenological theory of relativistic or non-relativistic many-body systems, Hilbert space of an isolated system must also carry a unitary representation of Poincaré or Galilei group. Then, the so-called cluster separability is a locality assumption, see, e.g., Refs. [38] or [18] and references therein. It is a condition on interaction terms in the generators of the space-time symmetry group saying: if the system is separated into disjoint subsystems (=clusters) by a sufficiently large spacelike separation, then each subsystem behaves as an isolated system with a suitable representation of space-time symmetries on its Hilbert space, see Ref. [38, Sec. 6.1. Let's call this principle cluster separability I.

Another special case of locality assumption has been described by Peres, Ref. [56], p. 128. Let us reformulate it as follows 
Cluster Separability II No quantum experiment with a system in a local laboratory is affected by the mere presence of an identical system in remote parts of the universe.

It is well known (see, e.g., Ref. [56], p. 136) that this principle leads to restrictions on possible statistics (fermions, bosons). What is less well known is that it also motivates non-trivial locality conditions on observables.

The locality condition is formulated in Ref. [56], p. 128:

... a state $w$ is called remote if $\|A w\|$ is vanishingly small, for any operator A which corresponds to a quantum test in a nearby location. ... We can now show that the entanglement of a local quantum system with another system in a remote state (as defined above) has no observable effect.

This is a condition on A inasmuch as there has to be at least one remote state for A.

However, Peres does not warn that the standard operators of quantum mechanics, which are in fact generators of space-time symmetries, do not satisfy his condition on A [31]. Similarly, basic observables of relativistic-field or many-body theories are generators of Poincaré or Galilei groups and so they do not satisfy the locality condition, either. It follows that cluster separability II is logically independent from the cluster decomposition or of cluster separability I. Of course, this does not mean that the basic observables are to be rejected. They are very useful if the assumption of isolated system is a good approximation. However, this assumption is definitely a bad one for quantum theory of measurement.

The present section reformulates and extends Peres' ideas. Let us explain everything, working in $Q$-representation of Hilbert space $\mathbf{H}_{\tau}$ and of operators on it. Then, one can also write tensor products as ordinary products and indicate the order of factors by indices at system coordinates.

Consider two experiments. In Experiment I, vector state $22\left(\vec{x}_{1}\right)$ of particle $\mathcal{S}_{1}$ of type $\tau$ is prepared in our laboratory. In Experiment II, state $\psi\left(\vec{x}_{1}\right)$ of particle $\mathcal{S}_{1}$ is prepared as in Experiment I and vector state $\phi\left(\vec{x}_{2}\right)$ of system $\mathcal{S}_{2}$ of the same type is prepared simultaneously in a remote laboratory. Then, according to Proposition 12 , the state of the composite system $\mathcal{S}$ must be

$$
\Psi\left(\vec{x}_{1}, \vec{x}_{2}\right)=\nu\left(\psi\left(\vec{x}_{1}\right) \phi\left(\vec{x}_{2}\right)+\epsilon(\tau) \phi\left(\vec{x}_{1}\right) \psi\left(\vec{x}_{2}\right)\right),
$$

where $\nu=\left[2\left(1+\epsilon|c|^{2}\right)\right]^{-1 / 2}$ is a normalisation factor, $c=\langle\psi \mid \phi\rangle$ while $\epsilon(\tau)$ is 1 for bosons and -1 for fermions.

\footnotetext{
${ }^{2}$ We let out the variable $\xi$ because it will not play any role in the subsequent discussion.
} 
Now, our lab claims that the state of $\mathcal{S}_{1}$ is $\psi\left(\vec{x}_{1}\right)$ because this state has been prepared in the lab according to all rules of experimental art. However, somebody who knows what has happened in both labs claims that the preparation has been a combination of two sub-preparations and that the state is (2.7). Hence, the notion of preparation and state becomes ambiguous, at least if Rule 12 holds without any restrictions. This is true even if states $\psi$ and $\phi$ are localized (the wave functions have supports) within the respective laboratories.

Localisation of the states $\psi$ and $\phi$ removes at least the following difficulty. Suppose that a fermion is prepared in a distant laboratory in a state $\phi$. Then a fermion of the same type cannot be prepared in the same state $\psi=\phi$ in our laboratory: the composite state of the two fermions had then to be zero (Pauli exclusion principle). However, if the wave function of the fermion prepared in our laboratory falls off rapidly outside our laboratory and that in the remote laboratory does the same outside the remote one, then the two wave functions would be different, $\psi(x) \neq \phi(x)$, and their antisymmetric combination would not be zero even if $\psi$ was just a Euclidean group transform of $\phi$. The requirement of the fall-off is very plausible indeed. It would be technically impossible to prepare a state with the same wave function $\psi$ in a laboratory located in Prague, and simultaneously in a laboratory in Bern, say, even for bosons.

Let us return to the ambiguity of preparations. It would not be really harmful if it had no measurable consequences. But it apparently has! Suppose that the registration on $\mathcal{S}_{1}$ corresponding to an observable a with kernel $a\left(\vec{x}_{1} ; \vec{x}_{1}^{\prime}\right)$ of $\mathcal{S}_{1}$ is performed in our laboratory after Experiment II. It is then equally possible that the registration is made on $\mathcal{S}_{1}$ or $\mathcal{S}_{2}$ and both can make a contribution to the outcome. Hence, the correct observable $A$ that is associated with such a registration is described by kernel

$$
A\left(\vec{x}_{1}, \vec{x}_{2} ; \vec{x}_{1}^{\prime}, \vec{x}_{2}^{\prime}\right)=a\left(\vec{x}_{1} ; \vec{x}_{1}^{\prime}\right) \delta\left(\vec{x}_{2}-\vec{x}_{2}^{\prime}\right)+a\left(\vec{x}_{2} ; \vec{x}_{2}^{\prime}\right) \delta\left(\vec{x}_{1}-\vec{x}_{1}^{\prime}\right)
$$

which is an operator on $\mathbf{H}_{\mathcal{R}(\tau)}^{2}$ so that it satisfies Proposition 12.

The average of a in the state prepared in Experiment $\mathrm{I}$ is $\langle\psi \mid \mathrm{a} \psi\rangle$. On the other hand, Experiment II leads to the average:

$$
\langle\Psi \mid \mathrm{A} \Psi\rangle=\frac{\langle\psi \mid \mathrm{a} \psi\rangle+\langle\phi \mid \mathrm{a} \phi\rangle+\epsilon(\tau) c\langle\phi \mid \mathrm{a} \psi\rangle+\epsilon(\tau) c^{*}\langle\psi \mid \mathrm{a} \phi\rangle}{1+\epsilon(\tau)|c|^{2}} .
$$

The two averages are different and the difference need not be small. For example, let the supports of the wave functions $\psi$ and $\phi$ be disjoint, each inside an open set around the corresponding laboratory. Then, $c=0$. Consider the position operator of $\mathcal{S}_{1}$. The kernel is $a\left(\vec{x}_{1} ; \vec{x}_{1}^{\prime}\right)=\vec{x}_{1} \delta\left(\vec{x}_{1}-\vec{x}_{1}^{\prime}\right)$, and the difference between the two averages becomes

$$
\int d^{3} x_{1} \vec{x}_{1} \phi^{*}\left(\vec{x}_{1}\right) \phi\left(\vec{x}_{1}\right)
$$


This can be made arbitrary large by shifting the remote laboratory sufficiently far away.

Thus, cluster separability is violated. However, the position does not satisfy Peres' condition: such an 'observable' controls position of the system in the whole infinite space. By that, it is utterly different from observables that can be registered in a human laboratory. It can analogously be shown that all standard operators of quantum mechanics are similar to position in this property.

\subsubsection{D-local states and observables}

This section is a more detailed and accurate account of the conditions on states and observables than that given by Peres. They will be assumed as at least approximately satisfied.

We shall consider general $N$-particle systems and only those operators A that fulfil the following condition. The kernel $A\left(\vec{x}_{1}, \cdots, \vec{x}_{N} ; \vec{x}_{1}^{\prime}, \cdots, \vec{x}_{N}^{\prime}\right)$ of $\mathrm{A}$ is a distribution on Schwartz space (its elements are rapidly decreasing $C^{\infty}$ functions, see, e.g., Ref. [63]) $\mathbf{S}\left(\mathbb{R}^{3 N}\right)$ in variables $\vec{x}_{1}^{\prime}, \cdots, \vec{x}_{N}^{\prime}$ for any fixed value of $\vec{x}_{1}, \cdots, \vec{x}_{N}$ such that

$$
g\left(\vec{x}_{1}, \cdots, \vec{x}_{N}\right)=\int d^{3 N} x^{\prime} A\left(\vec{x}_{1}, \cdots, \vec{x}_{N} ; \vec{x}_{1}^{\prime}, \cdots, \vec{x}_{N}^{\prime}\right) f\left(\vec{x}_{1}^{\prime}, \cdots, \vec{x}_{N}^{\prime}\right) \in \mathbf{S}\left(\mathbb{R}^{3 N}\right)
$$

for any $f\left(\vec{x}_{1}, \cdots, \vec{x}_{N}\right)$. This is usually satisfied, see Sec. 1.3.1.

Definition 12 Let $A\left(\vec{x}_{1}, \cdots, \vec{x}_{N} ; \vec{x}_{1}^{\prime}, \cdots, \vec{x}_{N}^{\prime}\right)$ and $B\left(\vec{x}_{1}, \cdots, \vec{x}_{N} ; \vec{x}_{1}^{\prime}, \cdots, \vec{x}_{N}^{\prime}\right)$ be two kernels. Then, for any $K=0,1, \cdots, N$,

$$
\int d^{3} x_{k_{1}}^{\prime} \cdots x_{k_{K}}^{\prime} A\left(\vec{x}_{1}, \cdots, \vec{x}_{N} ; \vec{x}_{1}^{\prime}, \cdots, \vec{x}_{N}^{\prime}\right) B\left(\vec{x}_{1}^{\prime}, \cdots, \vec{x}_{N}^{\prime} ; \vec{x}_{1}^{\prime \prime}, \cdots, \vec{x}_{N}^{\prime \prime}\right)
$$

is a distribution acting on test functions of $\mathbf{S}\left(\mathbb{R}^{6 N-3 K}\right)$ that maps test function $f\left(\vec{x}_{1}^{\prime}, \cdots, \vec{x}_{N}^{\prime}, \vec{x}_{1}^{\prime \prime}, \cdots, \vec{x}_{N}^{\prime \prime}\right)^{\Delta \prime}$ on test function $g\left(\vec{x}_{1}, \cdots, \vec{x}_{N}, \vec{x}_{1}^{\prime}, \cdots, \vec{x}_{N}^{\prime}\right)^{\Delta \prime}$, where symbol $(\cdots)^{\Delta \prime}$ means that $K$ variables $\vec{x}_{k_{1}}^{\prime}, \cdots, \vec{x}_{k_{K}}^{\prime}$ are left out from $N$ primed variables $\vec{x}_{1}^{\prime}, \cdots, \vec{x}_{N}^{\prime}$. Distribution (2.9) is called convolution $\mathrm{A} * \mathrm{~B}$ over variables $\vec{x}_{k_{1}}, \cdots, \vec{x}_{k_{K}}$.

Let our laboratory takes an open set $D \subset \mathbb{R}^{3}$ and similarly $D^{\prime}$ for the remote one. The change of $\mathcal{S}_{1}$ state due to some actions in a remote laboratory would not be measurable in our laboratory if the states and observables are $D$-local in the following sense:

Definition 13 Let $D \subset \mathbb{R}^{3}$ be open, let $\mathrm{A}$ be an operator of $\mathcal{S}$ and let the following conditions hold:

1. $A\left(\vec{x}_{1}, \cdots, \vec{x}_{N} ; \vec{x}_{1}^{\prime}, \cdots, \vec{x}_{N}^{\prime}\right)$ is the zero distribution for any $\vec{x}_{1}, \cdots, \vec{x}_{N} \in \mathbb{R}^{3 N} \backslash$ $D^{N}$. 
2.

$$
\int d^{3 N} x^{\prime} A\left(\vec{x}_{1}, \cdots, \vec{x}_{N} ; \vec{x}_{1}^{\prime}, \cdots, \vec{x}_{N}^{\prime}\right) f\left(\vec{x}_{1}^{\prime}, \cdots, \vec{x}_{N}^{\prime}\right)=0
$$

for any test function $f$ such that

$$
\operatorname{supp} f\left(\vec{x}_{1}^{\prime}, \cdots, \vec{x}_{N}^{\prime}\right) \subset \mathbb{R}^{3 N} \backslash D^{N} .
$$

Then A is called D-local.

Let A be $D$-local for some open set $D$, let $D^{\prime}$ be open and $D \subset D^{\prime}$. Then, A is $D^{\prime}$-local. Let A be $D$-local and also $D^{\prime}$-local for two open sets $D$ and $D^{\prime}$. Then, A is $\left(D \cap D^{\prime}\right)$-local. Thus, all open sets $D$ such that $\mathrm{A}$ is $D$-local form a filter in the Boolean lattice of open subsets of $\mathbb{R}^{3}$.

Example $1 N=1, A\left(\vec{x} ; \vec{x}^{\prime}\right)=x^{1} \delta\left(\vec{x}-\vec{x}^{\prime}\right)$. A is $\mathbb{R}^{3}$-local and the filter is $\mathbb{R}^{3}$.

Example $2 N=1, \psi(\vec{x})$ is a wave function with support $D$. Then $|\psi(\vec{x})\rangle\langle\psi(\vec{x})|$ is $D^{\prime}$-local, where $D^{\prime}$ is the interior of $D$, and the filter is the family of all open sets containing $D^{\prime}$.

Definition 14 A state with a D-local state operator $\mathrm{T}$ is called D-local. An observable $\mathrm{E}(X)$, all effects $\mathrm{E}(X), X \in \mathbf{F}$, of which are D-local, is called D-local.

The key property is the following:

Proposition 13 Let system $\mathcal{S}_{1}$ of type $\tau_{1}$ composite of $N_{1}$ particles (not necessarily identical) be prepared in state $\mathrm{T}_{1}$ that is $D_{1}$-local. Let system $\mathcal{S}_{2}$ of type $\tau_{2}$ composite of $N_{2}$ particles be prepared in state $\mathrm{T}_{2}$ that is $D_{2}$-local, where $D_{1} \cap D_{2}=\emptyset$. Then, the state of $\mathcal{S}_{1}+\mathcal{S}_{2}$ according to Rule 12 is

$$
\mathrm{P}\left(\mathrm{T}_{1} \otimes \mathrm{T}_{2}\right) \mathrm{P}
$$

where $\mathrm{P}$ is the symmetrisation over all identical bosonic and the anti-symmetrisation over all identical fermionic particles contained in $\mathcal{S}_{1}+\mathcal{S}_{2}$ followed by multiplication by a normalisation factor. Furthermore:

1. Let $\tau_{1}=\tau_{2}\left(N_{1}=N_{2}\right)$ and let $\mathrm{E}(X)$ be a D-local observable for $\mathcal{S}_{1}$ as well as for $\mathcal{S}_{2}$. Then,

$$
\operatorname{tr}\left[(\mathrm{E}(X) \otimes 1+1 \otimes \mathrm{E}(X)) \mathrm{P}\left(\mathrm{T}_{1} \otimes \mathrm{T}_{2}\right) \mathrm{P}\right]=\operatorname{tr}\left[\mathrm{E}(X) \mathrm{T}_{1}\right]
$$

2. Let $\tau_{1} \neq \tau_{2}$ and let $\mathrm{E}(X)$ be a D-local observable for $\mathcal{S}_{1}$. Then

$$
\operatorname{tr}\left[(\mathrm{E}(X) \otimes 1) \mathrm{P}\left(\mathrm{T}_{1} \otimes \mathrm{T}_{2}\right) \mathrm{P}\right]=\operatorname{tr}\left[\mathrm{E}(X) \mathrm{T}_{1}\right]
$$


Thus, the existence of identical systems somewhere else does not influence the registrations of $D$-local observables in our laboratory.

Proof First, we formulate an immediate consequence of the definition of $D$-locality and of convolution. Let $\mathrm{A}$ be $D$-local and $\mathrm{B} D^{\prime}$-local, where $D \cap D^{\prime}=\emptyset$. Then convolution $\mathrm{A} * \mathrm{~B}$ over any variables $\vec{x}_{k_{1}}, \cdots, \vec{x}_{k_{K}}$ for $K \neq 0$ is the zero distribution on $\mathbf{S}\left(\mathbb{R}^{6 N-3 K}\right)$.

Let us first prove Part 1 of the proposition. Then, the kernel of $\mathrm{E}(X) \otimes 1+1 \otimes \mathrm{E}(X)$ is

$$
\begin{aligned}
E(X)\left(\vec{x}_{1}, \cdots, \vec{x}_{N} ; \vec{x}_{1}^{\prime}, \cdots, \vec{x}_{N}^{\prime}\right) \delta\left(\vec{y}_{1}-\vec{y}_{1}^{\prime}\right) \cdots \delta\left(\vec{y}_{N}-\vec{y}_{N}^{\prime}\right) \\
+\delta\left(\vec{x}_{1}-\vec{x}_{1}^{\prime}\right) \cdots \delta\left(\vec{x}_{N}-\vec{x}_{N}^{\prime}\right) E(X)\left(\vec{y}_{1}, \cdots, \vec{y}_{N} ; \vec{y}_{1}^{\prime}, \cdots, \vec{y}_{N}^{\prime}\right)
\end{aligned}
$$

and that of $\mathrm{T}_{1} \otimes \mathrm{T}_{2}$ is

$$
T_{1}\left(\vec{x}_{1}^{\prime}, \cdots, \vec{x}_{N}^{\prime} ; \vec{x}_{1}^{\prime \prime}, \cdots, \vec{x}_{N}^{\prime \prime}\right) T_{2}\left(\vec{y}_{1}^{\prime}, \cdots, \vec{y}_{N}^{\prime} ; \vec{y}_{1}^{\prime \prime}, \cdots, \vec{y}_{N}^{\prime \prime}\right)
$$

Operator $\mathrm{P}$ from the left acts in three steps. First, it makes permutations of all variables inside each group of variables $\vec{x}_{1}^{\prime}, \cdots, \vec{x}_{N}^{\prime}, \vec{y}_{1}^{\prime}, \cdots, \vec{y}_{N}^{\prime}$ that correspond to one type of particles in expression (2.12). Second, it sums with the correct sign over all such permutations that result in different terms. Finally, it multiplies the resulting sum by a normalisation factor. Operator $\mathrm{P}$ from the right acts similarly on variables $\vec{x}_{1}^{\prime \prime}, \cdots, \vec{x}_{N}^{\prime \prime}, \vec{y}_{1}^{\prime \prime}, \cdots, \vec{y}_{N}^{\prime \prime}$.

Thus, the trace on the left-hand side of Eq. (2.10) is a sum of traces of all terms obtained in this way. Let one such term be

$$
\int d^{3} x_{1}^{\prime} \ldots d^{3} x_{N}^{\prime} d^{3} y_{1}^{\prime} \ldots d^{3} y_{N}^{\prime} E(X)\left(x ; x^{\prime}\right) \delta\left(y ; y^{\prime}\right) T_{1}(\cdots ; \cdots) T_{2}(\cdots ; \cdots)
$$

where we have indicated variables $\vec{x}_{1}^{\prime}, \cdots, \vec{x}_{N}^{\prime}$ by $x^{\prime}$, etc., and '. ' ' in arguments of $\mathrm{T}_{1}$ or $\mathrm{T}_{2}$ stands for $N$ variables from $\vec{x}_{1}^{\prime}, \cdots, \vec{x}_{N}^{\prime}, \vec{y}_{1}^{\prime}, \cdots, \vec{y}_{N}^{\prime}$ or from $\vec{x}_{1}^{\prime \prime}, \cdots, \vec{x}_{N}^{\prime \prime}, \vec{y}_{1}^{\prime \prime}, \cdots, \vec{y}_{N}^{\prime \prime}$ obtained by the corresponding permutations.

If the permutations only change the order of $x$ 's or of $y$ 's, then all such terms are equal and the value of the trace is

$$
\begin{array}{r}
\operatorname{tr}\left[\int d^{3} x_{1}^{\prime} \ldots d^{3} x_{N}^{\prime} d^{3} y_{1}^{\prime} \ldots d^{3} y_{N}^{\prime} E(X)\left(x ; x^{\prime}\right) \delta\left(y, y^{\prime}\right) T_{1}\left(x^{\prime} ; x^{\prime \prime}\right) T_{2}\left(y^{\prime} ; y^{\prime \prime}\right)\right] \\
=\operatorname{tr}\left[\mathrm{E}(X) \mathrm{T}_{1}\right]
\end{array}
$$

If, however, the permutations mix $K x$ 's with $K y$ 's, then the $6 N$-variables convolution contains a $K$-variables convolution $\mathrm{E}(X) * \mathrm{~T}_{2}$, which is zero. Hence, the contribution of all such terms is just $\operatorname{tr}\left[\mathrm{E}(X) \mathrm{T}_{1}\right]$. 
Next, consider the terms of the form

$$
\int d^{3} x_{1}^{\prime} \ldots d^{3} x_{N}^{\prime} d^{3} y_{1}^{\prime} \ldots d^{3} y_{N}^{\prime} \delta\left(x, x^{\prime}\right) E(X)\left(y: y^{\prime}\right) T_{1}(\cdots ; \cdots) T_{2}(\cdots ; \cdots),
$$

Analogously, only those permutations can contribute to the convolution that exchange all $x$ 's with all $y$ 's so that $E(X)\left(y: y^{\prime}\right)$ is convoluted with $T_{1}\left(y^{\prime} ; y^{\prime \prime}\right)$. This is possible because both systems are of the same type and so there is exactly one group of $y$ 's to each groups of $x$ 's and these two groups contain the same number of variables. Again, all such permutations give the same result and the total trace is, therefore, $2 \operatorname{tr}\left[\mathrm{E}(X) \mathrm{T}_{1}\right]$.

The normalisation factor is

$$
\left(\operatorname{tr}\left[\mathrm{P}\left(\mathrm{T}_{1} \otimes \mathrm{T}_{2}\right) \mathrm{P}\right]\right)^{-1}
$$

and it is clear that only those permutations contribute to the trace which do not lead to convolutions between $T_{1}$ and $T_{2}$. There are only two kinds of them: those mixing $x$ 's among themselves and $y$ 's among themselves, or those exchanging all $x$ 's with all $y$ 's. In each of the two cases, the trace is unity, hence the normalisation factor is $1 / 2$, which implies Eq. (2.10). The proof of Part 2 is similar but simpler, Q.E.D.

For example, if $|\psi\rangle\langle\psi|$ is $D$-local, $|\phi\rangle\langle\phi|$ is $D^{\prime}$-local and the $D$-local kernel $a_{D}\left(\vec{x}_{1}, \vec{x}_{1}^{\prime}\right)$ is used instead of $a\left(\vec{x}_{1} ; \vec{x}_{1}^{\prime}\right)$ in formula (2.8) defining operator $\mathrm{A}_{D}$ instead of $\mathrm{A}$ we obtain

$$
\begin{aligned}
\int_{D} d^{3} x_{1} \int_{D} d^{3} x_{1}^{\prime} \int_{D} d^{3} x_{2} \int_{D} d^{3} x_{2}^{\prime} \Psi^{*}\left(\vec{x}_{1}, \vec{x}_{2}\right) A_{D}\left(\vec{x}_{1}, \vec{x}_{1}^{\prime} ; \vec{x}_{2}, \vec{x}_{2}^{\prime}\right) \Psi\left(\vec{x}_{1}^{\prime}, \vec{x}_{2}^{\prime}\right) \\
=\int_{-\infty}^{\infty} d^{3} x_{1} \int_{-\infty}^{\infty} d^{3} x_{1}^{\prime} \psi^{*}\left(\vec{x}_{1}\right) a_{D}\left(\vec{x}_{1} ; \vec{x}_{1}^{\prime}\right) \psi\left(\vec{x}_{1}^{\prime}\right)
\end{aligned}
$$

as if no $\mathcal{S}_{2}$ existed. Thus, in this case, cluster separability holds.

However, the ambiguity of the state of $\mathcal{S}_{1}$ in Experiment II still remains: the state prepared in our laboratory is $|\psi\rangle\langle\psi|$ and, at the time that $\mathcal{S}_{2}$ is prepared, $\mathcal{S}_{1}$ loses its individual states if Rule 12 is valid without exceptions. This is clearly at variance with cluster separability; we shall remove this difficulty in Sec. 2.2.4.

Next, we shall prove that each observable $\mathrm{E}$ of system $\mathcal{S}$ of type $\tau$ is associated, for a reasonable open set $D$, with a unique $D$-local observable $\Lambda_{D}(\mathrm{E})$, the so-called $D$-localisation of $\mathrm{E}$, such that the condition

$$
\operatorname{tr}[\mathrm{E}(X) \mathrm{T}]=\operatorname{tr}\left[\Lambda_{D}(\mathrm{E}(X)) \mathrm{T}\right]
$$

is satisfied for all $X \in \mathbf{F}$ and for all $D$-local states $\mathbf{T}$. Thus, any standard observable $\mathrm{E}$ such as position, momentum, etc. can be registered in $D$ by registering $\Lambda_{D}(\mathrm{E})$ and the probability distribution would be the same as if we had registered $\mathrm{E}$. 
Let us denote by $\mathbf{H}_{\tau}(D)$ the Hilbert space obtained by completion of the linear space of $C^{\infty}$-functions with support in $D^{N}$ with respect to the inner product of $\mathbf{H}_{\tau}$. $\mathbf{H}_{\tau}(D)$ is a closed linear subspace of $\mathbf{H}_{\tau}$. Let $\mathrm{P}_{D}$ be the orthogonal projection from $\mathbf{H}_{\tau}$ onto $\mathbf{H}_{\tau}(D)$.

\section{Definition 15 Let}

$$
\Lambda_{D}: \mathbf{L}_{r}\left(\mathbf{H}_{\tau}\right) \mapsto \mathbf{L}_{r}\left(\mathbf{H}_{\tau}(D)\right)
$$

be defined by

$$
\Lambda_{D}(\mathrm{~A})=\mathrm{P}_{D} \mathrm{AP}_{D}
$$

Mapping $\Lambda_{D}$ is called $D$-localisation.

Clearly, D-localisation of any operator in $\mathbf{L}_{r}\left(\mathbf{H}_{\tau}\right)$ is Hermitean, bounded and positive, hence

$$
\Lambda_{D}: \mathbf{L}_{r}\left(\mathbf{H}_{\tau}\right)_{\leq 1}^{+} \mapsto \mathbf{L}_{r}\left(\mathbf{H}_{\tau}\right)_{\leq 1}^{+}
$$

Of course, the $D$-localisation is not a unitary map. For example, it does not preserve operator norm, but it holds

$$
\left\|\Lambda_{D}(\mathrm{~A})\right\| \leq\|\mathrm{A}\|
$$

The operators and their $D$-localisations are considered as acting on $\mathbf{H}_{\tau}$. $D$-local operators leave $\mathbf{H}_{\tau}(D)$ invariant and define, therefore, also operators on Hilbert space $\mathbf{H}_{\tau}(D)$.

Second, we can use these facts for a construction of $D$-local POV measure on $\mathbf{H}_{\tau}(D)$ from any observable $\mathbf{E}$ on $\mathbf{H}_{\tau}$ by $D$-localising the effects $\mathbf{E}(X)$. The normalisation condition becomes

$$
\Lambda_{D}\left(\mathrm{E}\left(\mathbb{R}^{n}\right)\right)=\mathrm{P}_{D} 1 \mathrm{P}_{D}=1_{D}
$$

where $n$ is the dimension of $\mathrm{E}$ (see Sec. 1.2.1). Hence, D-localisation of an observable on $\mathbf{H}_{\tau}$ need not be an observable on $\mathbf{H}_{\tau}$ but it is always one on $\mathbf{H}_{\tau}(D)$. Also, $D$ localisation of a projection will not be a projection in general and so a $D$-localisation of a PV measure need not be a PV measure. Let us call this construction $D$ localisation of POV measures. All $D$-local POV measures commute with spectral projections of position PV measures, $\mathrm{E}_{k}^{\overrightarrow{\mathrm{Q}}}(X)$, if $X \cap D=\emptyset$, where $\mathrm{E}_{k}^{\overrightarrow{\mathrm{Q}}}(X)$ are sharp position observables that are well defined for $\mathcal{S}$. For example, if $\mathcal{S}$ is composite of $N$ particles of different type, then $k=1, \cdots, N$ and $\mathrm{E}_{k}^{\overrightarrow{\mathrm{Q}}}(X)$ is a spectral projection of the position of the $k$-th particle. Thus, the restriction to $D$-local observables may be formally understood in terms of superselection rules (see Sec. 3.3).

If the map $\Lambda_{D}$ is involved in construction of $D$-local states, it must be followed by a suitable normalisation. A $D$-local state on $\mathbf{H}_{\tau}$ is a state on $\mathbf{H}_{\tau}(D)$ and vice versa. 


\subsubsection{Separation status}

With the help of $D$-local states and observables, we could remove some contradictions due to unrestricted validity of Rule 12 . The ambiguity of states, however, remained. This is a great and ubiquitous problem. Indeed, any microsystem $\mathcal{S}$ is always a subsystem of a huge family of identical subsystems that exist somewhere in the world.

If we could write down the state of this huge family in accordance with unrestricted Rule 12, then the results of any registrations in our laboratory would be well defined by it. However, such a state is not and cannot be known. Luckily, the results of registration of $D$-local observables do not depend on the unknown parts of the state because of Proposition 13. This is the reason why the validity of Rule 12 can and must be restricted.

Clearly, one could ignore the entanglement of a single microsystem $\mathcal{S}$ with all microsystems of the same type, if $\mathcal{S}$ had a non-trivial separation status in the following sense:

Definition 16 Let $D \subset \mathbb{R}^{3}$ be an open set and system $\mathcal{S}$ be prepared in state $\mathrm{T}$. Let the following conditions be satisfied:

- The probability to register value of observable $\mathrm{E}(X)$ in set $X$ be $\operatorname{tr}\left[\mathrm{T}^{\prime} \mathrm{E}(X)\right]$ for any D-local observable $\mathrm{E}(X)$ and for any state $\mathrm{T}^{\prime}$ of $\mathcal{S}$.

- There is at least one D-local observable $\mathrm{E}^{\prime}(X)$ such that $\operatorname{tr}\left[\mathrm{TE}^{\prime}(X)\right] \neq 0$ for some $X$.

Then, open set $D$ is called separation status of $\mathcal{S}$.

The first condition means that the registration of $\mathrm{E}(X)$ on $\mathrm{T}^{\prime}$ is not disturbed by any identical system in a state different from $T^{\prime}$. This includes the following condition that must be fulfilled by the apparatus $\mathcal{A}$ that registers observable $\mathrm{E}(X)$ : The states of those subsystems of $\mathcal{A}$ that are identical with $\mathcal{S}$ do not disturb the registration of $\mathrm{E}(X)$ by $\mathcal{A}$ (but may disturb other registrations). A model of $\mathcal{A}$ satisfying the condition is given in Sec. 6.3. We can then view $\mathcal{S}$ as a physical object with a modified set of observables. The modification depends on open set $D$ that is determined by a preparation of $\mathcal{S}$. This motivates the following

Rule 13 Effects of $\mathcal{S}$ that can be registered must lie inside subsets $\mathbf{L}_{r}\left(\mathbf{H}_{\tau}(D)\right)_{\leq 1}^{+} \subset$ $\mathbf{L}_{r}\left(\mathbf{H}_{\tau}\right)_{\leq 1}^{+}$, where $D$ is the separation status of $\mathcal{S}$.

Thus, strictly speaking, no quantum system is proper (see Sec. 1.2.2).

For example, a microsystem that is alone in the Universe has separation status $D=\mathbb{R}^{3}$. This is a form of the assumption that a system is isolated. Observables 
of such a system are the standard ones. The same microsystem in a open set $D$ which is surrounded by matter containing a lot of microsystems of the same type such that supports of their states do not intersect $D$ has separation status $D$ and its observables are the $D$-local ones. The trivial case of separation status for a microsystem is $D=\emptyset$. It has then no observables of its own.

The composition of systems of the same type is determined by Rule 12 and its exceptions that are specified as follows.

Rule 14 Let two systems, $\mathcal{S}$ and $\mathcal{S}^{\prime}$, have separation statuses $D \neq \emptyset$ and $D^{\prime} \neq \emptyset$, respectively so that $D \cap D^{\prime}=\emptyset$. Then, the compositions of $\mathcal{S}$ and $\mathcal{S}^{\prime}$ follow rules valid for composition of systems of different type even if $\mathcal{S}$ and $\mathcal{S}^{\prime}$ have in common some subsystems of the same type.

For instance, let the wave function of particle $\mathcal{S}$ with separation status $D$ be $\psi(\vec{x})$ and let $\mathcal{S}^{\prime}$ be a family of $N$ particles identical with $\mathcal{S}$. Let $\mathcal{S}^{\prime}$ have separation status $D^{\prime}$ such that $D^{\prime} \cap D=\emptyset$. Its state $\Psi\left(\vec{x}_{1}, \cdots, \vec{x}_{N}\right)$ is symmetric or anti-symmetric in its $N$ arguments according to the type. Then the wave function of composite system $\mathcal{S}+\mathcal{S}^{\prime}$ of $N+1$ particles of the same type must be written as

$$
\psi(\vec{x}) \Psi\left(\vec{x}_{1}, \cdots, \vec{x}_{N}\right)
$$

Observe that wave function (2.14) is not (anti-)symmetric in all $N+1$ arguments! This is at variance with unrestricted validity of Rule 12 . According to Rule 12, the wave function had to be

$$
\mathrm{P}_{\mathbf{S}} \mathrm{P}_{\tau}^{(N+1)}\left(\psi(\vec{x}) \Psi\left(\vec{x}_{1}, \cdots, \vec{x}_{N}\right)\right)
$$

where $\mathbf{P}_{\mathbf{S}}$ is the projection to the unit sphere of $\mathbf{H}_{\mathcal{R}(\tau)}^{N+1}$. Now, it also ought to be clear why we do not employ Fock-space method to deal with identical systems: it automatically (anti-)symmetrises over all systems of the same type.

Rule 14 is in complete agreement with the common practice in quantum mechanics. For example, in the theory of the experiment described in Sec. 0.1.2, a state is prepared as a state of an individual electron and its entanglement with all other electrons, which exist, in fact, everywhere in huge amounts, is serenely ignored. Due to Proposition 13, the results of such method would not lead to any contradictions.

Let us give a mathematical example of separation status change. As for states, let it consist of the transformation of state (2.14) into state (2.15). This transformation has the form

$$
\mathrm{P}_{\mathbf{S}} \circ \mathrm{P}_{s, a}^{(N+1)}: \mathbf{H} \otimes \mathbf{H}_{s, a}^{N} \mapsto \mathbf{H}_{s, a}^{N+1}
$$

which is a non-invertible and non-linear map between two different Hilbert spaces. As for observables, in state (2.14), the observables that are registrable on $\mathcal{S}$ form 
the algebra $\mathbf{A}_{D}$ of $D$-local operators. Indeed, for any $a_{D} \in \mathbf{A}_{D}$, there is observable $\mathrm{a}_{D} \otimes 1$ on $\mathcal{S}+\mathcal{S}^{\prime}$. In state (2.15), the algebra would be $\mathbf{A}_{\emptyset}=\emptyset$ : there are no observables whatsoever that would be registrable individually on $\mathcal{S}$. Thus, the set of observables changes from $\mathbf{A}_{D}$ to $\mathbf{A}_{\emptyset}$.

In classical mechanics, the possible states of system $\mathcal{S}$ are all positive normalised functions (distribution functions) on the phase space $\mathbf{P}$ and possible observables are all real function on $\mathbf{P}$ (at least, all such observables have definite averages on $\mathcal{S}$ independently of external circumstances). $\mathbf{P}$ is uniquely associated with the system alone and forms the basis of its kinematic description. Hence, transitions between different sets of observables similar to those described above would be impossible in classical mechanics. They are only enabled in quantum mechanics by the non-objective character of observables: not only their values cannot be ascribed to microsystem $\mathcal{S}$ alone but some of them are not even registrable in principle due to external conditions in which $\mathcal{S}$ is.

We assume that the quantum kinematics of a microsystem is defined mathematically by possible states represented by all positive normalised (trace one) operators, and possible observables represented by some POV measures, on the Hilbert space associated with the system. Then the transitions of states and observables that go with changes of separation status cannot be viewed as a part of a dynamical trajectory due to some new version of the dynamics of $\mathcal{S}$, but as a change of its kinematic description. Thus, although the change of separation status is similar to the collapse of the wave function (the non-local character included 3 ), it is more radical and its physical roots are better understood.

What has been said up to now shows that standard quantum mechanics is incomplete in the following sense:

1. It accepts and knows only two separation statuses:

(a) that of isolated systems, $D=\mathbb{R}^{3}$, with the standard operators as observables, and

(b) that of a member of a system of identical particles, $D=\emptyset$, with no observables of its own.

2. It provides no rules for changes of separation status.

Let us call these theories fixed status quantum mechanics (FSQM). Our main idea is: Quantum mechanics must be supplemented by a theory of general separation status and by new rules that govern processes in which separation status changes. The

\footnotetext{
${ }^{3}$ The time instant, when such a non-local change takes place, can in some cases be established by observations 37 .
} 
new rules must not contradict the rest of quantum mechanics and ought to agree with and explain observational facts. 


\section{Chapter 3}

\section{Further general ideas}

This chapter contains further important general ideas and closes the account of the general language of quantum mechanics. It explains the relation between the ontological and epistemic aspects of probability notion. It describes some crucial properties of quantum observables such as joint measurability and contextuality. Finally, it introduces the notion of superselection rules in order to make clear that our approaches to classical properties and quantum measurement have noting to do with the superselection-rules one.

\subsection{Probability and information}

Quantum mechanics is a statistical theory. Thus, a correct understanding of probability and information is an important part in the conceptual framework of the theory. The discussion whether probability describes objective properties that can be observed in nature or subjective state of the knowledge of some human has raged since the invention of probability calculus by Jacob Bernoulli and PierreSimon Laplace [36]. The origin of the problem might be that the dispute cannot be decided: probability has both aspects, ontological and epistemic.

Probability is a function of a proposition $A$ and its value, $p(A)$, is a measure of the degree of certainty that $A$ is true. As a function on a Boolean lattice of propositions, it satisfies Cox' axioms [36]. Then it becomes a real additive measure on the lattice. Whether a proposition is true or false must be decided by observation, at least in principle. Hence, the probability always concerns objective events, at least indirectly.

As an example, consider the state $T$ of a quantum object $\mathcal{S}$ that has been prepared and a registration of an observable $\mathrm{E}$ on the state. The probability $p$ 志 $(X)$ concerns the proposition that the value of $E$ individually registered on $T$ lies in the set $X$. The value of the probability can be verified by studying the events that happen during 
such registrations. Thus, the probability concerns both the lack of knowledge of what objectively happens and properties of real systems. It is in this sense that probability has both epistemic and ontological aspects.

In quantum mechanics, $p_{\mathrm{\top}}^{\mathrm{E}}(X)$ is not the probability that $\mathrm{E}$ possesses a value within $X$ in the state $\mathrm{T}$ but the probability that a registration outcome will give such a value. The only exception is that $T$ is a gemenge of E-eigenstates. A related question is whether the outcomes are predictable and we just have not enough information to make the prediction, or objectively unpredictable. Quantum mechanics does not deliver the prediction and it would even be incompatible with any 'deeper' theory that did (see Sec. 3.2.2).

Let us describe the general framework that is necessary for any application of probability theory. First, there is a system, denote it by $\mathcal{S}$. In the example above, there are certain conditions that are imposed on the quantum object $\mathcal{S}$. State $\mathrm{T}$ is prepared: this is a definite objective condition on $\mathcal{S}$. Observable $\mathrm{E}$ is registered: another objective condition on $\mathcal{S}$. In general, there must always be an analogous set of conditions, let us denote it by $\mathcal{C}$. To each system $\mathcal{S}$ subject to condition $\mathcal{C}$ possibilities in some range are open. These possibilities are described by a set of propositions that form a Boolean lattice $\mathbf{F}$. A probability distribution $p: \mathbf{F} \mapsto[0,1]$ is a real additive measure on $\mathbf{F}$.

If $E$ is a discrete observable, its value set $\Omega$ is at most countable, $\Omega=\left\{\omega_{1}, \omega_{2} \cdots\right\}$. Then the single-element sets $\left\{\omega_{k}\right\}, k=1,2, \cdots$, are atoms of $\mathbf{F}$ that generate $\mathbf{F}$ and the probability distribution $p_{\mathrm{\top}}^{\mathrm{E}}(X)$ can be calculated from $p_{\mathrm{\top}}^{\mathrm{E}}\left(\left\{\omega_{k}\right\}\right)$ by means of Cox' axioms. The atoms are called outcomes. If $\mathrm{E}$ is a sharp continuous observable, then there are no atoms but continuous observables can be considered as idealisations of more realistic POV measures with well-defined atoms. (The reading scale of each registration apparatus can be divided into separate finite intervals, the width of which corresponds to the accuracy that can really be achieved, etc. see, e.g., Ref. [16], p. 35.)

If condition $\mathcal{C}$ is reproducible or it obtains spontaneously sufficiently often, anybody can test the value of the probabilities. The probability distribution $p(X)$ is therefore an objective property of condition $\mathcal{C}$ on $\mathcal{S}$. It is so even in cases when the outcomes can be predicted uniquely. This would depend on whether condition $\mathcal{C}$ can be sharpened to other conditions $\mathcal{C}_{k}$ so that

1. whenever $\mathcal{C}_{k}$ is satisfied, so is $\mathcal{C}$,

2. each $\mathcal{C}_{k}$ is still recognisable and reproducible and

3. each outcome allowed by $\mathcal{C}$ is uniquely determined by one of $\mathcal{C}_{k}$ 's.

Even in such a case, condition $\mathcal{C}$ itself leaves the system a definite amount of freedom that can be described in all detail by the probability distribution $p(X)$ : it 
is an objective, verifiable property of $\mathcal{C}$ alone. And, if we know only that condition $\mathcal{C}$ obtains and that a probability distribution is its objective property, then this probability distribution describes the state of our knowledge, independently of whether the conditions $\mathcal{C}_{k}$ do exist and we just do not know which of them obtains in each case or not. There is, therefore, no contradiction between the objective and subjective aspects of probability.

Entropy is a certain functional of $p(X)$ that inherits both objective and subjective aspects from probability. Discussions similar to those about probability spoil the atmosphere about entropy. The existence of a subjective aspect of entropy - the lack of information - seduces people to ask confused questions such as: Can a change of our knowledge about a system change the system energy?

The general definition of entropy as a measure of missing information has been given by Shannon [66] and its various applications to communication theory are, e.g., described in the book by Pierce [57]. Our version is:

Definition 17 Let $p: \mathbf{F} \mapsto[0,1]$ be a probability distribution and let $X_{k}$ be the atoms of $\mathbf{F}$. Then the entropy of $p(X)$ is

$$
S=-\sum_{k} p\left(X_{k}\right) \ln \left(p\left(X_{k}\right)\right)
$$

Let us return to the quantum-mechanical example in order to explain which information is concerned. After the choice of preparation and registration devices, we do not know what will be the outcome of a registration but we just know that any outcome $X_{k}$ of the registration has probability $p_{\mathrm{\top}}^{\mathrm{E}}\left(X_{k}\right)$. After an individual registration, one particular outcome will be known with certainty. The amount of information gained by the registration is the value of $S$ given by Eq. (3.1). For more detail, see Ref. [56]. Thus, the value of $S$ measures the lack of information before the registration.

The entropy and the so-called Maximum Entropy Principle1 (MEP) have become important notions of mathematical probability calculus, see, e.g., Ref. [36]. The mathematical problem MEP solves can be generally characterised as follows. Let system $\mathcal{S}$, condition $\mathcal{C}$ and lattice $\mathbf{F}$ with atoms $X_{k}$ be given. Let there be more than one set of $p\left(X_{k}\right)$ 's that is compatible with $\mathcal{C}$. How the probabilities $p\left(X_{k}\right)$ are to be assigned so that condition $\mathcal{C}$ is properly accounted for without any additional bias? Such $p\left(X_{k}\right)$ 's yield the maximum of $S$ as given by (3.1). MEP clearly follows from the meaning of entropy as a measure of lack of information. We shall use this kind of MEP.

\footnotetext{
${ }^{1}$ There is also a principle of statistical thermodynamics that carries the same name but ought not to be confused with the mathematical MEP.
} 
In quantum mechanics, probability distributions are generally associated with both states and observables. This has to do with the non-objective nature of observables. Still, can there be a general measure of information lack associated with a given state alone? Clearly, such a question is meaningful and the answer is the minimum of entropies, each associated with the state and an observable. The minimum, $S(\mathrm{~T})$ is a well-defined function of state $\mathrm{T}$ and is called von Neumann entropy. One can show (see, e.g., Ref. [56]) that, up to a constant factor,

$$
S(\mathrm{~T})=-\operatorname{tr}[\mathrm{T} \ln (\mathrm{T})]
$$

As each $\mathrm{T}$ must have a discrete spectrum with positive eigenvalues $t_{k}$, we have

$$
S(\mathrm{~T})=-\sum_{k} t_{k} \ln \left(t_{k}\right)
$$

The lack of information $S(\mathrm{~T})$ is associated with the state $\mathrm{T}$ of object $\mathcal{S}$ and consequently with the (classical) condition $\mathcal{C}$ that defines the preparation. Hence, according to our criterion of objectivity, von Neumann entropy is an objective property of object $\mathcal{S}$ prepared in state $\mathrm{T}$.

MEP together with the von Neumann entropy can be applied to thermodynamics. Consider the problem: what is the state $T$ that maximises von Neumann entropy under the condition that it has a given average $\operatorname{tr}[\mathrm{TE}]$ of energy (this is an objective property in our approach)? The answer is: the Gibbs' state and the value of Lagrange multiplier that multiplies the average of energy in the variational principle is $1 / k T_{B}$, where $T$ is the temperature and $k_{B}$ Boltzmann constant (for proof see, e.g. [56]).

Von Neumann entropy of composite objects satisfies a number of interesting inequalities, see, e.g., Ref.[71]. One is the so-called sub-additivity [2].

Proposition 14 Let $\mathcal{S}$ be composed of objects $\mathcal{S}_{1}$ and $\mathcal{S}_{2}$ of different types. Let $\mathrm{T}$ be the state of $\mathcal{S}$ and let

$$
\mathrm{T}_{1}=\Pi_{2}(\mathrm{~T}), \quad \mathrm{T}_{2}=\Pi_{1}(\mathrm{~T}) .
$$

Then

$$
S(\mathrm{~T}) \leq S\left(\mathrm{~T}_{1}\right)+S\left(\mathrm{~T}_{2}\right)
$$

and the equality sign is valid only if

$$
\mathrm{T}=\mathrm{T}_{1} \otimes \mathrm{T}_{2}
$$

Thus, von Neumann entropy can be used to measure the amount of entanglement in a state of a composite object. 


\subsection{Joint measurability and contextuality}

Joint measurability is related to contextuality by the mathematics used to describe each. Joint measurability is defined by a map from a Boolean lattice into the lattice of effects, and contextuality is related to maps from the sublattice of projections into the Boolean lattice $\{0,1\}$.

\subsubsection{Joint measurability}

Let $A$ and $B$ be two s.a. operators on Hilbert space $\mathbf{H}$ that are not necessarily bounded. Then the product $A B$ as well as the operators $A B+B A$ and $A B-B A$ are well defined on their common invariant domain $\mathbf{D}$ (see Sec. 1.3.1). The latter operator (or its extension) is called commutator of $A$ and $B$ and is denoted by $[A, B]$.

Proposition 15 Let $\mathrm{A}$ and $\mathrm{B}$ be two s.a. operators with a common invariant domain, let $i[\mathrm{~A}, \mathrm{~B}]$ have a s.a. extension and let $\mathrm{T}$ be an arbitrary state. Then

$$
\Delta \mathrm{A} \Delta \mathrm{B} \geq \frac{|\langle[\mathrm{A}, \mathrm{B}]\rangle|}{2}
$$

Equation (3.3) is called uncertainty relation.

The interpretation of the uncertainty relation is as follows. If we prepare many copies of an object in state $\mathrm{T}$ and register either $\mathrm{A}$ or $\mathrm{B}$ or $i[\mathrm{~A}, \mathrm{~B}]$ on each, then the average of $[A, B]$ and variances of $A$ as well as of $B$ will satisfy Eq. (3.3). It is not necessary to register all observables jointly.

An important notion of quantum mechanics is that of joint measurability. Often, it is also called simultaneous measurability but the notion has nothing to do with time. It simply means that there is one registration device that can measure both quantities.

Definition 18 Two elements $\mathrm{E}_{1}$ and $\mathrm{E}_{2}$ of $\mathbf{L}_{r}(\mathbf{H})_{\leq 1}^{+}$are jointly measurable if there is a POV measure $\mathrm{E}: \mathbf{F} \mapsto \mathbf{L}_{r}(\mathbf{H})_{\leq 1}^{+}$such that $\mathrm{E}_{1}=\mathrm{E}\left(X_{1}\right)$ and $\mathrm{E}_{2}=\mathrm{E}\left(X_{2}\right)$ for some $X_{1}$ and $X_{2}$ in $\mathbf{F}$.

This is a mathematical property that, in fact, is only a necessary condition for existence of the required registration device. Even if $\mathrm{E}$ existed, the question whether it is an observable is non-trivial.

Proposition 16 Two effects $\mathrm{E}_{1}$ and $\mathrm{E}_{2}$ are jointly measurable if and only if there are three elements $\mathrm{E}_{1}^{\prime}, \mathrm{E}_{2}^{\prime}$ and $\mathrm{E}_{12}^{\prime}$ in $\mathbf{L}_{r}(\mathbf{H})_{\leq 1}^{+}$such that

$$
\mathrm{E}_{1}^{\prime}+\mathrm{E}_{2}^{\prime}+\mathrm{E}_{12}^{\prime} \in \mathbf{L}_{r}(\mathbf{H})_{\leq 1}^{+}
$$


and

$$
\mathrm{E}_{1}=\mathrm{E}_{1}^{\prime}+\mathrm{E}_{12}^{\prime}, \quad \mathrm{E}_{2}=\mathrm{E}_{2}^{\prime}+\mathrm{E}_{12}^{\prime} .
$$

For proof, see [48], p. 89. Proposition 16 has two corollaries: 1) two projections are jointly measurable if they commute and 2) a sufficient condition for joint measurability of $E_{1}$ and $E_{2}$ is that

$$
\mathrm{E}_{1}+\mathrm{E}_{2} \in \mathbf{L}_{r}(\mathbf{H})_{\leq 1}^{+} .
$$

If an observable is sharp then its effects commute, but the effects of a general observable need not commute. Thus, even non commuting operators can be jointly measurable. This is completely compatible with standard quantum mechanics. Indeed, any POV measure that is an observable of an object $\mathcal{S}$ can be measured as a sharp observable of a composite system containing $\mathcal{S}$ and another object called ancilla as subsystems. For proof, see e.g. [56], p. 285. An important example will be given in Sec. 4.4 .

Two observables $\mathrm{E}_{1}: \mathbf{F}_{1} \mapsto \mathbf{L}_{r}(\mathbf{H})$ and $\mathrm{E}_{2}: \mathbf{F}_{2} \mapsto \mathbf{L}_{r}(\mathbf{H})$ with $\mathbf{F}_{1}$ comprising the Borel subsets of $\mathbb{R}^{n_{1}}$ and $\mathbf{F}_{2}$ those of $\mathbb{R}^{n_{2}}$ are called jointly measurable, if each two effects $\mathrm{E}_{1}\left(X_{1}\right)$ and $\mathrm{E}_{2}\left(X_{2}\right)$ are jointly measurable. In this case, there is an effect $\mathrm{E}_{1}\left(X_{1}\right) \wedge \mathrm{E}_{2}\left(X_{2}\right)$ in $\mathbf{L}_{r}(\mathbf{H})_{\leq 1}^{+}$giving the probability that observable $\mathbf{E}_{1}$ has value in $X_{1}$ and observable $\mathrm{E}_{2}$ has value in $X_{2}$. Then, there is a unique observable

$$
\mathrm{E}: \mathbf{F} \mapsto \mathbf{L}_{r}(\mathbf{H})
$$

where $\mathbf{F}$ is the set of Borel subsets of $\mathbb{R}^{n_{1}+n_{2}}$ and

$$
\mathrm{E}\left(X_{1} \times X_{2}\right)=\mathrm{E}_{1}\left(X_{1}\right) \wedge \mathrm{E}_{2}\left(X_{2}\right)
$$

We call $\mathrm{E}$ compound of $\mathrm{E}_{1}$ and $\mathrm{E}_{2}$

An important example are two sharp observables A and B. They are jointly measurable, if and only if all their effects (which are projections) commute with each other, i.e., the operators commute. In this case, the wedge is just the ordinary operator product,

$$
\mathrm{E}^{\mathrm{A}}\left(X_{1}\right) \wedge \mathrm{E}^{\mathrm{B}}\left(X_{2}\right)=\mathrm{E}^{\mathrm{A}}\left(X_{1}\right) \mathrm{E}^{\mathrm{B}}\left(X_{2}\right) .
$$

The compound $E^{A \wedge B}$ of $E^{A}$ and $E^{B}$ is an observable that represents registration of a pair of values, one of $A$, the other of $B$.

If two sharp observables $A$ and $B$ do not commute then they can be jointly measurable at best with the inaccuracy corresponding to their uncertainty relation. This means that only some effects of A are jointly measurable with only some effects of B. An example will be given in Sec. 4.4. 


\subsubsection{Contextuality}

The investigations in the field of contextuality were motivated by the following problem. In Newton mechanics, a general state of any system $\mathcal{S}$ is described by probability distributions $\rho: Z \mapsto[0,1], Z \in \boldsymbol{\Gamma}$ on its phase space $\boldsymbol{\Gamma}$. Any such distribution results from a fixed preparation and describes the ensemble of individual systems $\mathcal{S}$ prepared in this way. Each individual system of the ensemble is, however, always assumed, at least in Newton mechanics, to be in some state given by a point $Z$ of $\boldsymbol{\Gamma}$. We just do not know which point and the distribution $\rho(Z)$ describes the state of our incomplete knowledge. Thus, all observables, which are just functions on $\boldsymbol{\Gamma}$, have determinate values for each element of the ensemble.

Can anything analogous be also assumed for quantum mechanics? Could a state operator of a quantum object $\mathcal{S}$ describe our knowledge of an ensemble defined by the preparation and could the individual elements of the ensemble each have determinate values of all observables, which are just not known, or even, be it for whatever reason, could not be known? To solve this problem, it is sufficient to restrict the observables to sharp observables, because if the question had negative answer for a restricted class, it would have negative answer for any class containing the restricted one.

It is technically advantageous to restrict the problem even further by limiting oneself to (perpendicular) projection operators to subspaces of $\mathbf{H}_{\tau}$. Let us denote the set of all such projections by $\mathbf{P}\left(\mathbf{H}_{\tau}\right) \subset \mathbf{L}_{r}\left(\mathbf{H}_{\tau}\right)$. The problem can then be formulated as follows. Is there a dispersion-free probability distribution $h: \mathbf{P}\left(\mathbf{H}_{\tau}\right) \mapsto\{0,1\}$ ? Dispersion-free means that its values are just zero and one so that such a distribution determines values of all projections.

The dispersion-free distribution $h$ has to fulfil certain conditions, or else it could not be interpreted as concerning properties. Let us first describe the structure of $\mathbf{P}\left(\mathbf{H}_{\tau}\right)$.

Each projection a $\in \mathbf{P}\left(\mathbf{H}_{\tau}\right)$ can be mapped on linear subspace $\mathbf{a}\left(\mathbf{H}_{\tau}\right)$ of $\mathbf{H}_{\tau}$ and this bijective map allows to define the lattice relations on $\mathbf{P}\left(\mathbf{H}_{\tau}\right)$. First, $\mathrm{a} \leq \mathrm{b}$ if $\mathrm{a}\left(\mathbf{H}_{\tau}\right) \subset \mathrm{b}\left(\mathbf{H}_{\tau}\right)$. This defines a partial ordering on $\mathbf{P}\left(\mathbf{H}_{\tau}\right)$. Second, $\mathrm{a}=\mathrm{b}^{\perp}$ if $\mathrm{a}\left(\mathbf{H}_{\tau}\right)$ contains all vectors orthogonal to $\mathrm{b}\left(\mathbf{H}_{\tau}\right)$. Projection a is called the orthocomplement of $b$. Third, $c=a \wedge b$ if $c\left(\mathbf{H}_{\tau}\right)$ is the set-theoretical intersection of $a\left(\mathbf{H}_{\tau}\right)$ and $b\left(\mathbf{H}_{\tau}\right)$. Observe that $\mathrm{a} \wedge \mathrm{b}=\mathrm{ab}$ (operator product of the projections) only if $\mathrm{a}$ and $\mathrm{b}$ commute (are orthogonal). Finally, $\mathrm{c}=\mathrm{a} \vee \mathrm{b}$ if $\mathrm{c}\left(\mathbf{H}_{\tau}\right)$ is the linear hull of $\mathrm{a}\left(\mathbf{H}_{\tau}\right)$ and $\mathrm{b}\left(\mathbf{H}_{\tau}\right)$.

$\mathbf{P}\left(\mathbf{H}_{\tau}\right)$ with these relations forms the so-called orthocomplemented lattice (for proof, see, e.g., Refs. [9, 15]), but not a Boolean lattices. It has however Boolean

\footnotetext{
${ }^{2}$ In the so-called quantum logic, properties of $\mathcal{S}$ are described by elements of $\mathbf{P}\left(\mathbf{H}_{\tau}\right)$. They represent the mathematical counterpart of the so-called YES-NO registrations [58. If we pretend that the values obtained in the YES-NO experiments are properties of a well-defined single
} 
sublattices, which represent sets of jointly measurable sharp effects, and must, therefore contain only mutually commuting projectors. On these sublattices, $h$ is, in fact, an assignment of truth values 0,1 and it has to satisfy the usual logical conditions

$$
h(\mathrm{a} \vee \mathrm{b})=h(\mathrm{a}) \vee h(\mathrm{~b}), \quad h(\mathrm{a} \wedge \mathrm{b})=h(\mathrm{a}) \wedge h(\mathrm{~b}), \quad h\left(\mathrm{a}^{\perp}\right)=h(\mathrm{a})^{\perp} .
$$

In these relations, we consider the set $\{0,1\}$ as a Boolean lattice of one empty set and one arbitrary non-empty set $\mathbf{A}$ with $\perp$ the set-theoretical complement in $\mathbf{A}$, $\checkmark$ the set-theoretical union and $\wedge$ the set-theoretical intersection. Thus, on each Boolean sublattice, $h$ must also be a Boolean lattice homomorphism.

The first result relevant to the question of existence of such maps is the Gleason's theorem [25]3. It states that the set of all probability distributions on $\mathbf{P}\left(\mathbf{H}_{\tau}\right)$ is just $\mathbf{T}\left(\mathbf{H}_{\tau}\right)_{1}^{+}$. Thus, our dispersion-free distributions are to be found in $\mathbf{T}\left(\mathbf{H}_{\tau}\right)_{1}^{+}$. It is easy to show that there are none there.

One can object that $\mathbf{P}\left(\mathbf{H}_{\tau}\right)$ is an idealisation containing an infinity of observables. This leads to the question whether there is a finite subset of $\mathbf{P}\left(\mathbf{H}_{\tau}\right)$ that does not admit such distributions, either. This is the next relevant result, Kochen-Specker no-go theorem [40, in which an example of such a subset is given. There are more examples now provided by different physicists (see, e.g., Ref. [15]). The reason why $h$ does not exist is that the assignment of a truth value to projector a, say, depends on to which Boolean sublattice a belongs. This can be understood as 'context': in one case, a is registered jointly with elements of one Boolean sublattice, in another case with those of another sublattice.

The definitive result in this field is Bub-Clifton-Goldstein theorem [15] which lists all maximal sub-lattices of $\mathbf{P}\left(\mathbf{H}_{\tau}\right)$ that admit dispersion-free probability distributions. They need not be Boolean but they are always only proper sublattices of $\mathbf{P}\left(\mathbf{H}_{\tau}\right)$. Hence, only a limited number of properties can be assumed to be determinate before registration, and this limits possible 'non-collapse' interpretations and modifications of quantum mechanics. In fact, these interpretations or modifications can be classified according to the sublattices determined by the Bub-CliftonGoldstein theorem [15].

\subsection{Superselection rules}

A preparation that deals only with systems of one and the same type prepares so called one-type systems. Not every preparation is such. For example, we can ran-

quantum system, then we are forced to replace the Boolean lattice of ordinary logic by the orthocomplemented latice of quantum logic. But this pretence is against all logic because these values are not properties of one but of many different systems each consisting of $\mathcal{S}$ plus some registration apparatus.

${ }^{3}$ Gleason's theorem is valid for Hilbert spaces of dimensions greater than 2 . 
domly mix electrons in some states with protons in some states. Such systems, and some generalisations of them, are called mixed system 4 . In this section, the mathematical description of mixed systems will be explained. We shall mix just two one-type systems, $\mathcal{S}_{1}$ and $\mathcal{S}_{2}$, but the generalisation to any number is straightforward.

Let $\mathbf{H}_{1}$ and $\mathbf{H}_{2}$ be the Hilbert spaces of $\mathcal{S}_{1}$ and $\mathcal{S}_{2}$. Then the Hilbert space $\mathbf{H}$ of the system $\mathcal{S}$ mixing $\mathcal{S}_{1}$ and $\mathcal{S}_{2}$ is

$$
\mathbf{H}=\mathbf{H}_{1} \oplus \mathbf{H}_{2}
$$

The direct sum on the right-hand side is the space of pairs, $\left(\psi_{1}, \psi_{2}\right), \psi_{1} \in \mathbf{H}_{1}$ and $\psi_{2} \in \mathbf{H}_{2}$ with linear superposition defined by

$$
a\left(\psi_{1}, \psi_{2}\right)+b\left(\phi_{1}, \phi_{2}\right)=\left(a \psi_{1}+b \phi_{1}, a \psi_{2}+b \phi_{2}\right)
$$

and the inner product defined by

$$
\left\langle\left(\psi_{1}, \psi_{2}\right),\left(\phi_{1}, \phi_{2}\right)\right\rangle=\left\langle\psi_{1}, \phi_{1}\right\rangle_{1}+\left\langle\psi_{2}, \phi_{2}\right\rangle_{2}
$$

where $\langle\cdot, \cdot\rangle_{i}$ is the inner product of $\mathbf{H}_{i}$. There are then embeddings $\iota_{i}: \mathbf{H}_{i} \mapsto \mathbf{H}$ defined by

$$
\iota_{1}\left(\psi_{1}\right)=\left(\psi_{1}, 0\right), \quad \iota_{2}\left(\psi_{2}\right)=\left(0, \psi_{2}\right)
$$

for any $\psi_{i} \in \mathbf{H}_{i}$ and $i=1,2$.

Let $\mathbf{L}(\mathbf{H})$ be the algebra of bounded linear operators on $\mathbf{H}$ and $\mathbf{L}\left(\mathbf{H}_{i}\right)$ that of $\mathbf{H}_{i}$. If two operators $\mathrm{A}_{i} \in \mathbf{L}\left(\mathbf{H}_{i}\right), i=1,2$, are given, then an operator $\left(\mathrm{A}_{1} \oplus \mathrm{A}_{2}\right) \in \mathbf{L}(\mathbf{H})$, called direct sum of $A_{1}$ and $A_{2}$, is defined by

$$
\left(\mathrm{A}_{1} \oplus \mathrm{A}_{2}\right)\left(\psi_{1}, \psi_{2}\right)=\left(\mathrm{A}_{1}\left(\psi_{1}\right), \mathrm{A}_{2}\left(\psi_{2}\right)\right)
$$

for any $\left(\psi_{1}, \psi_{2}\right) \in \mathbf{H}$. The special property of the direct-sum operators is that the subspaces $\iota_{i}\left(\mathbf{H}_{i}\right) \subset \mathbf{H}$ are invariant with respect to them. Clearly, not all operators in $\mathbf{L}(\mathbf{H})$ are of this form. Effects of the form $A_{1} \oplus 0_{2}\left(0_{1} \oplus A_{2}\right)$ can be interpreted as representing registrations done on $\mathcal{S}_{1}\left(\mathcal{S}_{2}\right)$ alone.

The embeddings $\iota_{i}$ define maps on projections $\mathrm{P}\left[\psi_{i}\right]$, which can be denoted by the same symbol, viz.

$$
\iota_{i}\left(\mathrm{P}\left[\psi_{i}\right]\right)=\mathrm{P}\left[\iota_{i}\left(\psi_{i}\right)\right]
$$

for all $\psi_{i} \in \mathbf{H}_{i} . \quad \iota_{i}$ can be extended to the whole spaces $\mathbf{T}\left(\mathbf{H}_{i}\right)_{1}^{+}, \iota_{i}: \mathbf{T}\left(\mathbf{H}_{i}\right)_{1}^{+} \mapsto$ $\mathbf{T}(\mathbf{H})_{1}^{+}$as follows. Let $\mathrm{T}_{i} \in \mathbf{T}\left(\mathbf{H}_{i}\right)_{1}^{+}$, then

$$
\iota_{1}\left(\mathrm{~T}_{1}\right)=\left(\mathrm{T}_{1}, 0\right), \quad \iota_{2}\left(\mathrm{~T}_{2}\right)=\left(0, \mathrm{~T}_{2}\right) .
$$

\footnotetext{
${ }^{4}$ This has nothing to do with the term 'mixed state', which is sometimes used for non-vector states.
} 
The convex combinations of states from $\mathbf{T}\left(\mathbf{H}_{1}\right)_{1}^{+}$and $\mathbf{T}\left(\mathbf{H}_{2}\right)_{1}^{+}$are states of mixed systems defined at the beginning of this section. However, the states $\mathbf{T}(\mathbf{H})_{1}^{+}$are not exhausted by convex combinations of states from $\mathbf{T}\left(\mathbf{H}_{1}\right)_{1}^{+}$and $\mathbf{T}\left(\mathbf{H}_{2}\right)_{1}^{+}$.

The structural properties in which the systems $\mathcal{S}_{1}$ and $\mathcal{S}_{2}$ differ from each other can now be viewed as non-trivial operators on $\mathbf{H}$. Let for example the masses $\mu_{1}$ and $\mu_{2}$ satisfy $\mu_{1} \neq \mu_{2}$. Then the s.a. operator $\mu_{1} 1_{1} \oplus \mu_{2} 1_{2}$ defines the mass operator $\mathrm{m}$ on $\mathbf{H}$ with two eigenspaces $\iota_{i}\left(\mathbf{H}_{i}\right)$ and eigenvalues $\mu_{i}, i=1,2$. Similarly for charges, spins, etc. Now, it is easy to see that all operators of $\mathbf{L}(\mathbf{H})$ that commute with $\mathrm{m}$ have the form $A_{1} \oplus A_{2}$ and all operators of this form commute with m. Clearly, s.a. operators of the form $A_{1} \oplus A_{2}$ are observables of system $\mathcal{S}$. The nature of mixing systems suggests the basic rule

Rule 15 All effects that can be registered on system $\mathcal{S}$ mixing $\mathcal{S}_{1}$ and $\mathcal{S}_{2}$ have the form $\mathrm{A}_{1} \oplus \mathrm{A}_{2}$ where $\mathrm{A}_{1} \in \mathbf{L}_{r}\left(\mathbf{H}_{1}\right)_{\leq 1}^{+}$and $\mathbf{A}_{2} \in \mathbf{L}_{r}\left(\mathbf{H}_{2}\right)_{\leq 1}^{+}$.

Hence, the space of all observables of $\mathcal{S}$ is not $\mathbf{L}_{r}(\mathbf{H})_{\leq 1}^{+}$but the subset of all observables that commute with $\mathrm{m}$.

In general, we define

Definition 19 A discrete sharp observable $\mathbf{Z}$ of a system $\mathcal{S}$ with Hilbert space $\mathbf{H}$ is called superselection observable if $\mathbf{Z}$ commutes with all observables of $\mathcal{S}$, sharp or not sharp. The existence of such an observable is called superselection rule. The eigenspaces of $\mathbf{Z}$ are called superselection sectors. All superselection observables form the centre $\mathbf{Z}$ of the algebra $\mathbf{L}(\mathbf{H})$.

A restriction of the set of observables for system $\mathcal{S}$ suggests the introduction of an equivalence relation on the set of states $\mathbf{T}\left(\mathbf{H}_{\tau}\right)_{1}^{+}$.

Definition 20 Two states $\mathrm{T}_{1}$ and $\mathrm{T}_{2}$ are equivalent with respect to a set $\mathbf{O}$ of observables if these states assign the same probability measures to each observable of $\mathbf{O}$, that is , $p_{\mathrm{T}_{1}}^{\mathrm{E}}=p p_{\mathrm{T}_{2}}^{\mathrm{E}}$ for each $\mathrm{E} \in \mathbf{O}$. In that case, we write $\mathbf{T}_{1} \cong_{\mathbf{O}} \mathbf{T}_{2}$.

For example, linear superposition

$$
\Psi=a\left(\psi_{1}, 0\right)+b\left(0, \psi_{2}\right)
$$

for any $\psi_{i} \in \mathbf{H}_{i}, i=1,2,|a|^{2}+|b|^{2}=1$, and convex combination

$$
\mathbf{T}=|a|^{2} \iota_{1}\left(\left|\psi_{1}\right\rangle\left\langle\psi_{1}\right|\right)+|b|^{2} \iota_{2}\left(\left|\psi_{2}\right\rangle\left\langle\psi_{2}\right|\right)
$$

are equivalent with respect to the set of superselection observables $\mathbf{O}$,

$$
|\Psi\rangle\langle\Psi| \cong_{\mathbf{O}} \top .
$$


For a given set of observables $\mathbf{O}, \cong_{\mathbf{O}}$ is, indeed, an equivalence relation on the set of states $\mathbf{T}(\mathbf{H})_{1}^{+}$. Let us denote the set of equivalence classes of states in $\mathbf{T}(\mathbf{H})_{1}^{+}$ with respect to the set of observables $\mathbf{O}$ by $\mathbf{T}_{\mathbf{O}}(\mathbf{H})_{1}^{+}$. No two states of the same class can be distinguished by any registrations. No two different states from $\mathbf{T}(\mathbf{H})_{1}^{+}$ are equivalent with respect to all observables unless the set of effects $\mathbf{O}$ is restricted.

Proposition 17 Given a system $\mathcal{S}$ with the sets $\mathbf{O}$ and $\mathbf{T}_{\mathbf{O}}(\mathbf{H})_{1}^{+}$of observables and state classes, respectively. Then the following statements are equivalent:

A Z is a superselection observable.

B Each state is equivalent to a unique convex combination of eigenstates of $\mathbf{Z}$.

See Ref. [16], p. 18.

Rule 16 The states of system $\mathcal{S}$ mixing $\mathcal{S}_{1}$ and $\mathcal{S}_{2}$ are the equivalence classes with respect to the set of observables $\mathbf{O}$ of the form $\mathrm{A}_{1} \oplus \mathrm{A}_{2}$. The unique convex combination of eigenstates of $\mathbf{Z}$ to which each element of $\mathbf{T}(\mathbf{H})_{1}^{+}$is equivalent describes the gemenge structure of the class.

This motivates attempts to utilise superselection rules for solution of the problem of classical properties and of quantum measurement [34, 6, 11, 65, 62. The formalism of this section follows Ref. [16] because it gives some meaning to the linear superpositions of states from different superselection sectors, which is used by some of the references mentioned. 


\section{Part II}

\section{The models}


In this part, the basic notions and rules of Part 1 will be employed to construct models of observed systems or at least of some aspects thereof. The relation between Part 1 and Part 2 within any theory is more or less loose. The general language is used to construct models but it does not determine which models are to be constructed. Indeed, it could be used also to work with arbitrary or even fancy models that need not have much in common with practical experience. The scientific practice is to encounter various systems in nature and to construct models of these observed systems in order to understand and predict their properties. We can also improve earlier constructed models in order that they include newly observed properties, etc.

The division of a physical theory into a general part and a model part may have some relevance to Popper's philosophy (cf. [59]). The first part is a kind of language that does not have any specific, numerical consequences. Only the models give specific, numerical values that can be compared with experimental results and observations. It seems therefore that the general part is practically not falsifiable but a specific model surely is. Thus, a failure of a theory can always be interpreted as a failure of some models. Two different reactions to such a falsification are possible. Either one constructs a completely new theory starting with the general part, or one tries to improve the models. For example, the difficulties of Newton mechanics in the domain of quantum phenomena can either lead to the invention of quantum mechanics or to the improvement of the corresponding Newton-mechanical models. Indeed, the Bohm-de Broglie pilot-wave theory can be viewed as an example of the latter approach.

The menagerie of successful models is a very important part of any physical theory. Textbooks of quantum mechanics dedicate most of their space to an account of it. We shall restrict ourself only to those models that are immediately important to our main aim: to solve the problem of classical properties and quantum measurement. 


\section{Chapter 4}

\section{Models of microscopic systems}

In this chapter, we limit ourselves just to few examples of microsystems, first to show the crucial steps of model construction within our conception of quantum mechanics and second to introduce some models that will be important later.

The construction of quantum models can be divided into two steps. First, assumptions are made about states and observables of particles. Second, the states and observables of a composite quantum system can be constructed as described in Secs. 2.1 and 2.2 in agreement with its structural properties such as composition and a specific Hamiltonian.

The operators that will be defined in the present section will have some relations to similar quantities that describe classical systems of an analogous composition and they will have names that do not seem to be justified by anything but this classical analogy. This is true but it will be interpreted in this paper as follows. The definition of the operators is a purely quantum-mechanical business and the role of the corresponding observables as well as their registration method belong to important new assumptions of the theory. The only concession we can do is that the classical mechanics can give us a hint on how quantum operators may be constructed, but the definitive decision is only possible if the model works, that is, predicts properly what can be observed.

One often tries to find some way from classical systems to analogous quantum systems, a so-called 'quantisation' method, or at least to formulate some kind of 'correspondence principle' concerning relations between classical and quantum mechanics. In our opinion, the natural way is just the opposite and must lead from quantum mechanics to the classical one because classical systems are just some macroscopic quantum systems. This ought to explain any correspondence.

Model assumptions are assumptions about specific systems that are to be modelled. They will, therefore, be called 'Assumptions' to distinguish them from general 'Rules'. 


\subsection{Basic PV measures}

Let us start with the following structural properties: a particle with mass $\mu$, spin $s$ and charge $e$ (this determines the type $\tau$ ). To construct the Hilbert space $\mathbf{H}_{\mu, s, e}$ of such model, we shall work in $Q$-representation. Consider the set of rapidly decreasing $C^{\infty}$ functions $\phi(\vec{x}, m)$ (wave functions), where $m \in\{-s, \cdots, s\}$. Then, $\mathbf{H}_{\mu, s, e}$ is the completion of the set of the wave functions with respect to the inner product

$$
\langle\phi \mid \psi\rangle=\sum_{m} \int_{\mathbb{R}^{3}} d^{3} x \phi^{*}(\vec{x}, m) \psi(\vec{x}, m) .
$$

The states of the particle that can be prepared are elements of $\mathbf{T}\left(\mathbf{H}_{\mu, s, e}\right)_{1}^{+}$.

Next, we define certain operators on $\mathbf{H}_{\mu, s, e}$ : position $\overrightarrow{\mathbf{q}}=\left(\mathbf{q}^{1}, \mathbf{q}^{2}, \mathbf{q}^{3}\right)$, (linear) momentum $\overrightarrow{\mathrm{p}}=\left(\mathrm{p}^{1}, \mathrm{p}^{2}, \mathrm{p}^{3}\right)$ and $\operatorname{spin} \overrightarrow{\mathrm{s}}=\left(\mathrm{s}^{1}, \mathrm{~s}^{2}, \mathrm{~s}^{3}\right)$, where the symbol $\overrightarrow{\mathrm{q}}$ denotes the compound observable (see Sec. 3.2.1) $q^{1} \wedge q^{2} \wedge q^{3}$ and similarly for other vectorial operators.

The position and momentum operators have already been defined in Sec. 1.3, Eqs. (1.21) and (1.22). The three components of spin are $(2 s+1) \times(2 s+1)$ Hermitean matrices $s_{m n}^{k}$ that act on wave functions (vector-valued functions) as follows

$$
\mathrm{s}^{k} \phi(\vec{x}, m)=\sum_{n=-s}^{s} s_{m n}^{k} \phi(\vec{x}, n) .
$$

In non relativistic quantum mechanics, one mostly works only with $s=0$ or $s=1 / 2$. The matrices for $s=1 / 2$ are

$$
\mathrm{s}^{1}=\hbar / 2\left(\begin{array}{ll}
0 & 1 \\
1 & 0
\end{array}\right), \quad \mathrm{s}^{2}=\hbar / 2\left(\begin{array}{cc}
0 & -i \\
i & 0
\end{array}\right), \quad \mathrm{s}^{3}=\hbar / 2\left(\begin{array}{cc}
1 & 0 \\
0 & -1
\end{array}\right) .
$$

All components of $\vec{q}, \vec{p}$ and $\vec{s}$ can be extended to self-adjoint (s.a.) operators on $\mathbf{H}_{\mu, s, e}$.

The common invariant domain of operators $\vec{q}, \vec{p}$ and $\vec{s}$ is the set of rapidly decreasing $C^{\infty}$ functions $\phi(\vec{x}, m)$. Hence one can construct other operators from them by products and linear combinations, e.g., orbital angular momentum

$$
\mathrm{L}^{1}=\mathrm{q}^{2} \mathrm{p}^{3}-\mathrm{q}^{3} \mathrm{p}^{2}, \quad \mathrm{~L}^{2}=\mathrm{q}^{3} \mathrm{p}^{1}-\mathrm{q}^{1} \mathrm{p}^{3}, \quad \mathrm{~L}^{3}=\mathrm{q}^{1} \mathrm{p}^{2}-\mathrm{q}^{2} \mathrm{p}^{1}
$$

The operator has a unique s.a. extension. One of the assumptions of quantum mechanics is that one can construct all observables for a particle from its basic operators. This is the reason why they are called basic. The construction is simple, if the resulting observable is a polynomial. More complicated functions can be constructed using spectral theorem. For example, any function $V(\overrightarrow{\mathrm{q}})$ can be defined by

$$
V(\overrightarrow{\mathrm{q}})=\int_{\mathbb{R}^{3}} V\left(\iota^{1}, \iota^{2}, \iota^{3}\right) d E^{\overrightarrow{\mathrm{q}}}
$$


Let us construct the PV measures $\mathrm{E}^{\vec{q}}, \mathrm{E}^{\vec{p}}$ and $\mathrm{E}^{\mathrm{s}^{3}}$. The dimension of the first two is 3 and $\mathbf{F}$ is the set of all Borel subsets of $\mathbb{R}^{3}$. We define

$$
\mathrm{E}^{\overrightarrow{\mathrm{q}}}(X) \psi(\vec{x}, m)=\chi[X](\vec{x}) \psi(\vec{x}, m)
$$

where $\chi[X]$ is the characteristic function of $X \in \mathbf{F}$, that is, $\chi[X](\vec{x})=1$ for $\vec{x} \in X$ and $\chi[X](\vec{x})=0$ otherwise. Clearly, the value set of $\mathbf{E}^{\vec{q}}$ is the whole of $\mathbb{R}^{3}$.

For $E^{\vec{p}}$, we define

$$
\begin{aligned}
\mathrm{E}^{\vec{P}}(X) \psi(\vec{x}, m)=(2 \pi \hbar)^{-3} \int d^{3} p \exp \left(\frac{i}{\hbar} \vec{p} \cdot \vec{x}\right) \chi[X](\vec{p}) & \\
& \int d^{3} x^{\prime} \exp \left(-\frac{i}{\hbar} \vec{p} \cdot \vec{x}^{\prime}\right) \psi\left(\vec{x}^{\prime}, m\right)
\end{aligned}
$$

where $\chi[X](\vec{p})=1$ for $\vec{p} \in X$ and $\chi[X](\vec{p})=0$ otherwise. The value set of $\mathrm{E}^{\overrightarrow{\mathrm{p}}}$ is the whole of $\mathbb{R}^{3}$.

The three components of spin cannot be combined to a single PV measure because they do not commute. Let us describe the third component $\mathrm{s}^{3}$. PV measure $\mathrm{E}^{\mathrm{s}^{3}}$ is one-dimensional and $\mathbf{F}$ is the set of Borel subsets of $\mathbb{R}$. We define

$$
\mathrm{E}^{\mathrm{s}^{3}}(X) \psi(\vec{x}, m)=\chi[X](m) \psi(\vec{x}, m)
$$

where $\chi[X](m)=1$ if $m \in X \cap\{-s, \cdots, s\}$ and $\chi[X](m)=0$ otherwise. $\mathrm{E}^{\mathrm{s}^{3}}$ is a discrete PV measures with value space $\{-s, \cdots, s\}$.

Let us summarize:

Assumption 1 Any particle with mass $\mu$, spin s and charge $e$ is a quantum system with Hilbert space $\mathbf{H}_{\mu, s, e}$. Its basic operators are position, momentum and spin, defined by Eqs. (1.21), (1.2.2), as well as (4.1) and (4.2) for particles with spin 1/2.

According to Sec. 2.2.2, these basic PV measures are not observables. To obtain observables from them, separation status $D$ in which we are going to prepare all particles is to be determined and $D$-localisation of the PV measures have to be constructed. We shall speak about measurement of basic observables understanding the so constructed POV measures.

Suppose that a free particle is prepared in $D$. Registration of the (smeared) position of the particle can e.g. be carried out by a small Geiger counter that is placed so that its sensitive volume $V$ is at a fixed position in $D$. If it counts at time $t$, then we know that the registered position of the quantum system were in $V$ at $t$. The momentum of the particle can be inferred from its energy because its Hamiltonian is a quadratic function of the momentum (see the next subsection), and from the direction of motion that is given, e.g., by apertures in screens. This functional 
relation between momentum and Hamiltonian is of course only approximate for $D$ localised quantities. The spin can be registered via the Stern-Gerlach apparatus because even neutral particles have a non-zero gyromagnetic ratio.

Finally, the basic operators of a composite system can be obtained from the basic operators of its subsystems. A prominent example in any textbook of quantum mechanics is the hydrogen atom. A possible model of it is composed of an electron and a proton. The Hilbert space of hydrogen is $\mathbf{H}_{e} \otimes \mathbf{H}_{p}$, where $\mathbf{H}_{e}$ and $\mathbf{H}_{p}$ are those of the electron and the proton. These are just $\mathbf{H}_{\mu, s, e}$ with suitable numerical values of $\mu, s$ and $e$. The basic operators are $\overrightarrow{\mathrm{q}_{e}} \otimes 1_{p}, \overrightarrow{\mathrm{p}_{e}} \otimes 1_{p}, \overrightarrow{\mathrm{s}_{e}} \otimes 1_{p}, 1_{e} \otimes \overrightarrow{\mathrm{q}_{p}}, 1_{e} \otimes \overrightarrow{\mathrm{p}_{p}}$, $1_{e} \otimes \overrightarrow{\mathrm{s}_{p}}$.

Further operators can be constructed by algebraic operations from the basic ones, and the corresponding observables then by $D$-localisation.

\subsection{Hamiltonian}

Assumption 2 Hamiltonian of any particle with mass $\mu$, charge $e$ and gyromagnetic ratio $g$ moving in classical electrostatic potential $V(\vec{x})$ and classical stationary magnetic field $\vec{B}(\vec{x})$ is

$$
\mathrm{H}=\frac{\mathrm{p}^{2}}{2 \mu}+e V(\overrightarrow{\mathrm{q}})-g \overrightarrow{\mathrm{s}} \cdot \vec{B}(\overrightarrow{\mathrm{q}})
$$

where the square means the squared norm of a 3-vector and '.' is the inner product of 3-vectors.

A textbook model of hydrogen atom consists of a spin-zero proton of mass $\mu_{p}$ and charge $e$ and a spin-zero electron of mass $\mu_{e}$ and charge $-e$. The Hamiltonian of the model is

$$
\mathrm{H}=\frac{\mathrm{p}_{e}^{2}}{2 \mu_{e}}+\frac{\mathrm{p}_{p}^{2}}{2 \mu_{p}}-\frac{e^{2}}{\left|\overrightarrow{\mathrm{q}_{e}}-\overrightarrow{\mathrm{q}_{p}}\right|} .
$$

For any quantum system $\mathcal{S}$ with Hamiltonian $\mathrm{H}$, the spectrum of the s.a. operator $\mathrm{H}$ is called energy spectrum of $\mathcal{S}$. The energy spectrum is thus a structural property. Systems can be recognised via their spectra. If light comes from far away in the space and the (possibly Doppler shifted) Balmer series is found in its spectrum, hydrogen must be somewhere there.

Kinetic energy of a free system can e.g. be measured by a proportional counter. In fact, what is measured in this way is a $D$-localisation of the total kinetic energy, which is a s.a. operator that forms a part of the Hamiltonian. For a particle, it is

$$
\frac{\mathrm{p}^{2}}{2 \mu}
$$

and the spectrum is $[0, \infty)$. For the hydrogen atom, we had first to separate the motion of its centre of mass from its internal degrees of freedom. An utterly different 
business is a measurement of a spectrum of a bound system. Spectra of bound systems are discrete and can be measured if large number of the same systems emits or absorbs electromagnetic radiation. This is an indirect measurement via scattering of photons in which the final 'kinetic' energy of photons is measured by a spectrometer.

\subsection{Galilean group}

A free particle is defined by $V(\overrightarrow{\mathrm{q}})=$ const and $\vec{B}(\overrightarrow{\mathrm{q}})=0$. It is an isolated quantum system $\mathcal{S}$ and Galilean group has, therefore, a unitary ray representation on $\mathbf{H}_{\mu, s . e}$, see Sec. 1.3. We can describe it by writing up its generators. From Sec. 1.3, we know already the form of space translations, space rotations and time translations even for systems that are not isolated. The additional assumption is

Assumption 3 For a particle (not necessarily free) with mass $\mu$ and spin s, the total angular momentum is $\mathrm{J}^{k}=\mathrm{L}^{k}+\mathrm{s}^{k}$. For a free particle, the boosts are generated by $\mathrm{b}^{k}=-\mu \mathrm{q}^{k}$.

All operators are written in the Schrödinger picture. The only group representatives where multipliers appear is multiplication of boosts, $\mathrm{U}(\vec{v})$, with space shifts $\mathrm{U}(\vec{a})$. This leads to 'anomalous' commutator,

$$
\left[\mathrm{b}^{k}, \mathrm{p}_{l}\right]=-i \hbar \mu \delta_{k l}
$$

It is easy to work out how the corresponding one-parameter groups act on wave function $\psi(\vec{x}, m)$ :

$$
\mathrm{U}(\vec{a}) \psi(\vec{x}, m)=\psi(\vec{x}-\vec{a}, m)
$$

and

$$
\mathrm{U}(\vec{v}) \psi(\vec{x}, m)=\exp \left(\frac{i}{\hbar} \mu \vec{v} \cdot \vec{x}\right) \psi(\vec{x}, m)
$$

Thus, the boost just shifts the momentum by $\mu \vec{v}$. For example, the average of momentum in the shifted state equals to that in the original one plus $\mu \vec{v}$. The operators $\mathrm{b}^{k}$ and $\mathrm{p}_{k}$ generate a subgroup of $\mathbf{G}$ called Heisenberg group.

\subsection{Joint measurement of position and momentum}

Existence of an observable that represents a joint measurement of position and momentum will play some role in Chap. 5. To this aim, we generalise the construction 
of such an observable for a simplified model that was first proposed in Ref. [1]. The model system $\mathcal{S}$ is a free one-dimensional spin-zero particle with position q and momentum p. The Hilbert space is $L^{2}(\mathbb{R})$ and the operators are defined by equations analogous to (1.21) and (1.22).

Operators $\mathrm{q}$ and $\mathrm{p}$ have an invariant common domain and their commutator is easily calculated to be

$$
[\mathrm{q}, \mathrm{p}]=i \hbar
$$

Hence, the joint measurement may be a problem.

The general construction of a non-trivial POV measure for system $\mathcal{S}$ introduces another system, ancilla, that forms a composite system with $\mathcal{S}$. Let our ancilla $\mathcal{A}$ be a similar particle with position $\mathrm{Q}$ and momentum $\mathrm{P}$. We work in $Q$-representation so that the Hilbert space of the composite system $\mathcal{S}+\mathcal{A}$ is $L^{2}(\mathbb{R}) \otimes L^{2}(\mathbb{R})$ which can be identified with $L^{2}\left(\mathbb{R}^{2}\right)$. Then, we have wave functions $\Psi(q, Q)$ and integral operators with kernels of the form $O\left(q, Q ; q^{\prime}, Q^{\prime}\right)$.

The dynamical variables $\mathrm{A}=\mathrm{q}-\mathrm{Q}$ and $\mathrm{B}=\mathrm{p}+\mathrm{P}$ of the composite system $\mathcal{S}+\mathcal{A}$ commute and can therefore be measured jointly. The value space of PV observable $\mathrm{E}^{\mathrm{A} \wedge \mathrm{B}}$ is $\mathbb{R}^{2}$ with coordinates $a$ and $b$ (see end of Sec. 3.2.1).

The next step is to smear $\mathrm{E}^{\mathrm{A} \wedge \mathrm{B}}$ to obtain a realistic POV meausre $\mathrm{E}_{k}$. Let us divide the $a b$ plane into disjoint rectangular cells $X_{k}=\left[a_{k-}, a_{k+}\right] \times\left[b_{k-}, b_{k+}\right]$ covering the entire plane. Each cell is centred at $\left(a_{k}, b_{k}\right), a_{k}=\left(a_{k+}+a_{k-}\right) / 2, b_{k}=\left(b_{k+}+b_{k-}\right) / 2$ and $S_{k}=\left(a_{k+}-a_{k-}\right)\left(b_{k+}-b_{k-}\right)$ is its area. Then,

$$
\mathrm{E}_{k}=\mathrm{E}^{\mathrm{A} \wedge \mathrm{B}}\left(X_{k}\right)=\mathrm{E}^{\mathrm{A}}\left(\left[a_{k-}, a_{k+}\right]\right) \mathrm{E}^{\mathrm{B}}\left(\left[b_{k-}, b_{k+}\right]\right) .
$$

The cells can be arbitrarily small.

The probability to obtain the outcome $k$ in state $\mathrm{T}$ of the composite system is

$$
\operatorname{tr}\left[\mathrm{E}_{k} \mathrm{\top}\right]=\int d q d Q d q^{\prime} d Q^{\prime} E_{k}\left(q, Q ; q^{\prime}, Q^{\prime}\right) T\left(q^{\prime}, Q^{\prime} ; q, Q\right) .
$$

We assume that the composite system $\mathcal{S}+\mathcal{A}$ is prepared in a factorised state

$$
\mathrm{T}=\mathrm{T}_{\mathcal{S}} \otimes \mathrm{T}_{\mathcal{A}}
$$

and express the probability (4.7) in terms of the state $\mathrm{T}_{\mathcal{S}}$. The action of the projections $\mathrm{E}^{\mathrm{A}}\left(\left[a_{k-}, a_{k+}\right]\right)$ and $\mathrm{E}^{\mathrm{B}}\left(\left[b_{k-}, b_{k+}\right]\right)$ on vector states of the form $\Psi(q, Q)$ is

$$
\mathrm{E}^{\mathrm{A}}\left(\left[a_{k-}, a_{k+}\right]\right) \Psi(q, Q)=\chi\left[a_{k-}, a_{k+}\right](q-Q) \Psi(q, Q),
$$

where

$$
\begin{aligned}
& \chi\left[a_{k-}, a_{k+}\right](x)=1 \quad \forall x \in\left[a_{k-}, a_{k+}\right], \\
& \chi\left[a_{k-}, a_{k+}\right](x)=0 \quad \forall x \operatorname{not} \in\left[a_{k-}, a_{k+}\right]
\end{aligned}
$$


and

$$
\begin{aligned}
\mathrm{E}^{\mathrm{B}}\left(\left[b_{k-}, b_{k+}\right]\right) \Psi(q, Q)= & (2 \pi \hbar)^{-2} \int d p d P d q^{\prime} d Q^{\prime} \\
& \exp \frac{i}{\hbar}\left[p\left(q-q^{\prime}\right)+P\left(Q-Q^{\prime}\right)\right] \chi\left[b_{k-}, b_{k+}\right](p+P) \Psi\left(q^{\prime}, Q^{\prime}\right),
\end{aligned}
$$

where

$$
\begin{aligned}
& \chi\left[b_{k-}, b_{k+}\right](x)=1 \quad \forall x \in\left[b_{k-}, b_{k+}\right], \\
& \chi\left[b_{k-}, b_{k+}\right](x)=0 \quad \forall x \operatorname{not} \in\left[b_{k-}, b_{k+}\right] .
\end{aligned}
$$

The trace (4.7) can be calculated in several steps as follows. First,

$$
\begin{array}{r}
\operatorname{tr}\left[\mathrm{E}_{k} \mathrm{~T}\right]= \\
\operatorname{tr}\left[\mathrm{E}^{\mathrm{A}}\left(\left[a_{k-}, a_{k+}\right]\right) \mathrm{E}^{\mathrm{B}}\left(\left[b_{k-}, b_{k+}\right]\right) \mathrm{T}\right]=\operatorname{tr}\left[\mathrm{E}^{\mathrm{B}}\left(\left[b_{k-}, b_{k+}\right]\right) \mathrm{TE}^{\mathrm{A}}\left(\left[a_{k-}, a_{k+}\right]\right)\right] \\
=(2 \pi \hbar)^{-2} \int d q d Q d p d P d q^{\prime} d Q^{\prime} \exp \frac{i}{\hbar}\left[p\left(q-q^{\prime}\right)+P\left(Q-Q^{\prime}\right)\right] \\
\chi\left[b_{k-}, b_{k+}\right](p+P) \chi\left[a_{k-}, a_{k+}\right](q-Q) T\left(q^{\prime}, Q^{\prime} ; q, Q\right) .
\end{array}
$$

Second, introduce new integration variables $q, a, p, b, q^{\prime}, a^{\prime}$,

$$
\begin{aligned}
& \operatorname{tr}\left[\mathrm{E}_{k} \mathrm{~T}\right]=(2 \pi \hbar)^{-2} \int d q d a d p d b d q^{\prime} d a^{\prime} \\
& \exp \frac{i}{\hbar}\left[p\left(q-q^{\prime}\right)+(b-p)\left(q-a-q^{\prime}+a^{\prime}\right)\right] \chi\left[b_{k-}, b_{k+}\right](b) \chi\left[a_{k-}, a_{k+}\right](a) T\left(q^{\prime}, q^{\prime}-a^{\prime} ; q, q-a\right) .
\end{aligned}
$$

Third, if the cells are sufficiently small, the integrands do not change appreciably inside the integration intervals of $a$ and $b$ so that they can be approximated as follows:

$$
\int d a d b \chi\left[a_{k-}, a_{k+}\right](a) \chi\left[b_{k-}, b_{k+}\right](b) f(a, b) \approx S_{k} f\left(a_{k}, b_{k}\right) .
$$

In this way, we obtain

$$
\begin{aligned}
\operatorname{tr}\left[\mathrm{E}_{k} \mathrm{~T}\right] \approx \frac{S_{k}}{(2 \pi \hbar)^{2}} \int d q d p d q^{\prime} d a^{\prime} & \\
& \quad \exp \frac{i}{\hbar}\left[\left(p\left(a_{k}-a^{\prime}\right)+b_{k}\left(q-q^{\prime}-a_{k}+a^{\prime}\right)\right] T\left(q^{\prime}, q^{\prime}-a^{\prime} ; q, q-a_{k}\right) .\right.
\end{aligned}
$$

But, fourth, the factor containing the integral over $p$ is a $\delta$-function,

$$
\int d p \exp \frac{i}{\hbar} p\left(a_{k}-a^{\prime}\right)=2 \pi \hbar \delta\left(a^{\prime}-a_{k}\right)
$$

Thus, we obtain,

$$
\operatorname{tr}\left[\mathrm{E}_{k} \mathrm{~T}\right] \approx \frac{S_{k}}{(2 \pi \hbar)^{2}} \int d q d q^{\prime} \exp \frac{i}{\hbar} b_{k}\left(q-q^{\prime}\right) T\left(q^{\prime}, q^{\prime}-a_{k} ; q, q-a_{k}\right) .
$$


Fifth, we use Eq. (4.8),

$$
\mathrm{T}\left(q^{\prime}, Q^{\prime} ; q, Q\right)=\mathrm{T}_{\mathcal{S}}\left(q^{\prime}, q\right) \mathrm{\top}_{\mathcal{A}}\left(Q^{\prime}, Q\right)
$$

with the result

Proposition 18 The probability of the outcome $k$ to be found in the factorised state (4.8) is given approximately by

$$
\operatorname{tr}\left[\mathrm{E}_{k} \mathrm{~T}\right] \approx \operatorname{tr}\left[\mathrm{T}_{\mathcal{S}} \frac{S_{k}}{(2 \pi \hbar)^{2}} \mathrm{\top}_{\mathcal{A}}\left[a_{k}, b_{k}\right]\right]
$$

where

$$
\mathrm{T}_{\mathcal{A}}\left[a_{k}, b_{k}\right]=\exp \frac{i}{\hbar} b_{k}\left(q-q^{\prime}\right) T\left(q^{\prime}, q^{\prime}-a_{k} ; q, q-a_{k}\right)
$$

is the state $\mathrm{T}_{\mathcal{A}}$ first shifted by $a_{k}$ and then boosted by $-b_{k}$. The approximation improves if the cells are smaller.

If the cells are sufficiently small, we have $q \approx Q+a_{k}$ and $p \approx b_{k}-P$. In this way, the coordinate of $\mathcal{S}$ is shifted by $a_{k}$ while the inverted momentum is shifted by $-b_{k}$ with respect to those of $\mathcal{A}$.

Eq. (4.9) shows that there is an 'effective' POV measure $\mathrm{E}_{\mathcal{S} k}$ for system $\mathcal{S}$ defined by

$$
\mathrm{E}_{\mathcal{S} k}=\frac{S_{k}}{(2 \pi \hbar)^{2}} \mathrm{~T}_{\mathcal{A}}\left[a_{k}, b_{k}\right]
$$

that yields the probability of the outcome $k$ of the above registration considered as a registration performed on $\mathcal{S}$.

The state $T_{\mathcal{A}}$ is completely arbitrary. To construct a useful quantity, one usually chooses a vector state in the form of a Gaussian wave packet (see, e.g., Ref. [56], p. 418),

$$
\Psi_{\sigma}=\left(\pi \sigma^{2}\right)^{-1 / 4} \exp \left(-\frac{Q^{2}}{2 \sigma^{2}}\right)
$$

Easy calculation yields

$$
\langle Q\rangle=\langle P\rangle=0, \quad \Delta Q=\frac{\sigma}{\sqrt{2}}, \quad \Delta P=\frac{\hbar}{\sigma \sqrt{2}}
$$

so that $\Psi_{\sigma}$ is a state of minimum uncertainty. If we shift $\Psi_{\sigma}$ by $a_{k}$ and then boost it by $-b_{k}$, we obtain

$$
\Psi_{\sigma}\left[a_{k}, b_{k}\right]=\left(\pi \sigma^{2}\right)^{-1 / 4} \exp \left(-\frac{\left(Q-a_{k}\right)^{2}}{2 \sigma^{2}}-\frac{i}{\hbar} b_{k} Q\right)
$$

which is the Gaussian packet concentrated at $\left(a_{k},-b_{k}\right)$,

$$
\langle Q\rangle=a_{k}, \quad\langle P\rangle=-b_{k}
$$


and

$$
\Delta Q=\frac{\sigma}{\sqrt{2}}, \quad \Delta P=\frac{\hbar}{\sigma \sqrt{2}} .
$$

As the Gaussian wave packet is uniquely determined by its averages and variances, we can interpret the observable $\mathrm{E}_{k}$ as giving the probability that the corresponding registration applied to state $T_{\mathcal{S}}$ detects the state of $\mathcal{S}$ with the averages and variances given by Eqs. (4.10) and (4.11). 


\section{Chapter 5}

\section{Quantum models of classical properties}

Quantum mechanics is a genuinely statistical theory. A well-defined repeatable preparation procedure defines an ensemble. Values of any observable obtained by repeated registrations on individual elements of the ensemble satisfy statistical laws that are given by quantum mechanics. Moreover, in general, values of observables are not objective, i.e., the individual elements of the ensemble cannot be assumed to possess determinate values of their observables before or independently of registrations and the results of individual registrations cannot be predicted.

On the other hand, basic physical theories that describe classical world, i.e. mechanics, electrodynamics, theory of gravitation, are objective and deterministic in character. This comprises three assumptions. First, the states are determined by values of observables and can be absolutely sharp (they contain no random elements) and, second, the sharp states are objective in the sense that any system always really is in one of them. Fuzzy states are also possible. They have the form of probability distributions of sharp states and are due to incomplete knowledge. Third, dynamical laws determine a unique evolution of all states.

More generally (i.e., even for thermodynamics), there is a criterion of objectivity, the so-called Modified Principle of Macroscopic Realism1 (MPMR) [29]:

1. A macroscopic system which has available to it two or more distinct classical states is at any given time in a definite one of those states.

\footnotetext{
${ }^{1}$ The original Principle of Macroscopic Realism has been formulated by Leggett [44. We have replaced Leggett's 'macroscopically distinct (quantum) states' by 'distinct classical states'. Indeed, if the 'macroscopically distinct (quantum) states' include pure quantum states, point 1 of Leggett's macroscopic realism violates the principle of superposition. Then, one has to assume that some as yet unknown phenomena exist at the macroscopic level which are not compatible with standard quantum mechanics (see, e.g., 44] and the references therein). However, no such phenomena have been observed.
} 
2. It is possible in principle to determine which of these states the system is in without any effect on the state itself or on the subsequent system dynamics.

To obtain classical theories as some limiting cases of quantum mechanics or to construct quantum models of classical properties is a difficult problem. As yet, only partial and incomplete solutions to this problem exist. Let us now briefly review the most popular approaches to the problem. The shortcomings of the approaches are well known [22, 5, 70]. We mention them only fleetingly. First, the quantum decoherence theory [76, 75] works only if certain observables concerning both the environment and the quantum system cannot be measured (see the analysis in [22, 15]). The deep reason is that one works with values of observables. Second, the theories based on coarse-grained operators [56, 60, 41]: the problem is the same as with the decoherence. For example, the Leggett-Garg inequality [41] is a condition for the validity of the principle of macroscopic realism that works with values of observables. Third, the Coleman-Hepp theory [34, 6, 11] and its modifications [65, 62. they are based on some particular theorems that hold for infinite systems but do not hold even approximately for finite ones (see the analysis in [6]).

If we turn from theory to experiment, we may notice that any well-founded scientific observation of classical properties always has a statistical form. A measurement or observation is only viewed as well understood, if it is given as an average with a variance. This fact does not by itself contradict the deterministic character of the corresponding theory. The usual excuse is that the observation methods are beset with inaccuracy but that improvement of techniques can lead to better and better results approaching the 'objective' values arbitrarily closely.

This popular excuse is clearly incompatible with the assumption that the classical world is only an aspect of a deeper quantum world and that each classical model is nothing but a kind of incomplete description of the underlying quantum system. If we assume such universality of quantum theory, then the statistical character of classical observational results must not only be due to inaccuracy of observational methods but also to genuine uncertainty of quantum origin. This point of view is originally due to Exner [21], p. 669, and Born [12] and will be adopted here as a starting point of our theory of classical properties.

\subsection{Modified correspondence principle}

Born-Exner assumption has quite radical consequences that are only seldom realised. First, the exactly sharp states and trajectories of classical theories are not objective. They do not exist in reality but are only idealisations. What does really exist are fuzzy states and trajectories. The objectivity of fuzzy states in classical world is a difficult point to accept and understand. Let us explain it in more detail. 
In quantum mechanics, the basis of objectivity of dynamical properties is the objectivity of the conditions that define preparation procedures. In other words, if a property is uniquely determined by a preparation, then it is an objective property. If we look closely, the only hindrance to try the same idea in classical theories is just the custom always to speak about initial data instead of preparations. An initial datum is nothing but a state, more or less sharp one. The question on how an exactly sharp state can come into being is ignored. This in turn seems justified by the hypothesis that sharp states are objective, that is, they just exist by themselves.

Thus, one has to accept that preparation procedures play the same basic role in the classical as in quantum physics. Then, the nature and form of necessary preparation procedures must be specified and the corresponding states described.

Our starting point was that all physical systems are quantum systems. Let us now make it more precise. There is one level of description (approximative model of some aspects of a physical system), which determines a classical system $\mathcal{S}_{c}$ and its classical properties for which quantum theory is not needed, namely the classical description. In addition, the same system can also be understood as a quantum system $\mathcal{S}_{q}$ such that the classical properties of $\mathcal{S}_{c}$ are some objective quantum properties of $\mathcal{S}_{q}$. This follows from the fact that all classical properties can be assumed to be objective without any danger of contradictions. $\mathcal{S}_{q}$ is a quantum model of $\mathcal{S}_{c}$.

The construction of quantum model $\mathcal{S}_{q}$ consists of the following points. 1) The composition of $\mathcal{S}_{q}$ must be defined. 2) The observables that can be measured on $\mathcal{S}_{q}$ are to be determined. On the one hand, this is a non-trivial problem: there are relatively strong restrictions on what observables of macroscopic systems can be measured (Secs. 2.2.2 and 6.2). On the other, as any observable is measurable only by a classical apparatus, the existence of such apparatuses is tacitly assumed from the very beginning. Quantum model $\mathcal{S}_{q}$ will thus always depend on some classical elements. This does not mean that classicality has been smuggled in because, in our approach, classical properties are specific quantum ones. 3) A Hamiltonian operator of the system must be set up. Finally, the known classical properties of $\mathcal{S}_{c}$ must be listed and each derived as an objective property of $\mathcal{S}_{q}$ from the three sets of assumptions above. This is a self-consistent framework for a non-trivial problem.

It follows that there must be some at least approximate relation between classical observables of $\mathcal{S}_{c}$ and quantum observables of $\mathcal{S}_{q}$ as well as between the classical states of $\mathcal{S}_{c}$ and quantum states of $\mathcal{S}_{q}$. The following model assumption on such a relation might be viewed as our version of Correspondence Principle, let us call it Modified Correspondence Principle (MCP).

Assumption 4 First, the state of classical system $\mathcal{S}_{c}$ in a given classical theory is always described by a set of n numbers $\left\{a_{1}, \cdots, a_{n}\right\}$ (state coordinates) that represent values of some classical observables. Let us assume that there are preparation proce- 
dures that make the fuzziness of real classical states negligible. This can be described by considering the values $\left\{a_{1}, \cdots, a_{n}\right\}$ as averages with variances $\left\{\Delta a_{1}, \cdots, \Delta a_{n}\right\}$ that are relatively small,

$$
\frac{\operatorname{Max}_{k}\left\{\Delta a_{k}\right\}}{\operatorname{Max}_{k}\left\{a_{k}\right\}} \ll 1
$$

Such states are the best we can achieve on the way to sharp classical states.

Second, we assume that there is a subset $\left\{\mathrm{a}_{1}, \cdots, \mathrm{a}_{n}\right\}$ of sharp observables of quantum system $\mathcal{S}_{q}$ and a state $\mathrm{T}$ such that

$$
\operatorname{tr}\left[\mathrm{T} \mathbf{a}_{k}\right]=a_{k}, \quad \sqrt{\operatorname{tr}\left[\mathrm{Ta}_{k}^{2}\right)-\left(\operatorname{tr}\left[\mathrm{T} \mathbf{a}_{k}\right]\right)^{2}}=\Delta a_{k} .
$$

All such states can form a large subset of $\mathbf{T}\left(\mathbf{H}_{\mathcal{S}_{q}}\right)_{1}^{+}$. Each of them can be considered as a quantum counterpart of the original classical state.

Third, there are fuzzy classical states with a probability distribution $\rho\left(a_{1}, \cdots, a_{n}\right)$ that either need not have negligible variances or need not be determined by its averages and variances, or both. However, we assume that every classical state of this kind results from a preparation procedure such that the resulting state $\mathbf{T}$ that can be interpreted as a gemenge of some states with small variances and that $\rho\left(a_{1}, \cdots, a_{n}\right)$ can be well approximated by the coefficients of such a gemenge.

Let us give an example. The classical state of a mass point in mechanics can be described by three coordinates $q^{k}=a_{k}$ and three momenta $p_{k}=a_{3+k}$, and the operator algebra of its quantum model contains the operators $\mathrm{q}^{k}=\mathrm{a}_{k}$ and $\mathrm{p}_{k}=\mathrm{a}_{3+k}$.

Of course, we can define a sensible classical state only for macroscopic systems so that their classical states have a huge number of quantum counterparts and in this way contain much less information than their quantum states. Each of the many quantum states satisfying the above equations can be viewed as representing one and the same classical state.

Clearly, MCP does not need the assumption that all observables from $\left\{\mathrm{a}_{1}, \cdots, \mathrm{a}_{n}\right\}$ commute with each other. Then, even if they themselves are not jointly measurable, their fuzzy values can be (see Sec. 4.4).

Moreover, it does not follow that each classical property is an average of a quantum operator. That would be false. We assume only that the classical state coordinates $a_{1}, \ldots, a_{n}$ can be chosen in such a way.

It is important to realise that MCP is just compatible with but does not imply MPMR. It suggests the way of how quantum models of classical properties can be constructed. In each such model, validity of MPMR ought to be achieved independently.

We emphasize that our approach to classical properties is very different from the well-known WKB approximation method. In particular, pure states such as 
coherent ones that have averages with small variances do not satisfy MPMR. Not only are pure states readily linearly superposed but also any quantum registration that were to find the parameters of a coherent state (a generalized measurement: positive operator valued measure) would strongly change the state (for a general argument, see Ref. [16], p. 32).

An interesting subset of classical properties of macroscopic system are the thermodynamic ones. They are important for us because quantum models of these properties are available. Moreover, quantum models of this kind of classical properties are compatible with Copenhagen interpretation [49]. Existing models based on statistical physics need one non-trivial assumption: the states of sufficiently small macroscopic systems that we observe around us are approximately states of maximum entropy. As it has been discussed in Sec. 3.1, entropy is an objective property of quantum systems because it is defined by their preparation. Thus, the validity of thermodynamics depends on the preparation conditions, or the origin, of observed macroscopic systems. The averages that result from the models based on the maximum-entropy assumption agree with observations and have small variances spontaneously. In this way, they comply with MCP. We describe a simplified model in the next section.

The physical foundations of thermodynamics are not yet well-understood but there are many ideas around. They might follow partially from logic (Bayesian approach, [36]) and partially from quantum mechanics (thermodynamic limit, 69, Vol. 4). Some very interesting models of how maximum entropy quantum states come into being are based on entanglement [23, 61, 47, 26]).

In any case, statistical methods can be generalised as follows [30]. We assume that the maximum entropy principle underlies all classical properties:

Assumption 5 An overwhelming part of macroscopic systems occur in quantum states that approximately maximize entropy under the condition of given averages of some quantum observables.

Let us briefly compare our approach with the theories mentioned at the beginning of this section. It starts with the idea that all classical properties of a macroscopic system $\mathcal{S}$ in a quantum state $\mathrm{T}$ are certain objective quantum properties of $\mathcal{S}$ in $\mathrm{T}$. Then, first, objective properties are quantum properties of all quantum systems and there is no question about how they emerge in quantum mechanics. This can avoid e.g. the artificial construction in the Coleman-Hepp approach. The new point is that they are considered as, and proved to be, objective [29]. Hence, second, they could in principle serve as classical properties because they can satisfy MPMR. This avoids the problems of both the quantum-decoherence and the coarse-grained theory that assume values of quantum observables to be objective. Such an assumption, as analysed in [15], works only for restricted classes of observables, all 
other measurements being forbidden without an independent justification. Third, we conjecture that certain macroscopic quantum systems possess objective properties, either structural or dynamical, that can fully model their classical behaviour. Hence, classical states and properties defined in the present paper are available only for some quantum systems and the relation between classical and quantum states is not one-to-one but one-to-many. This is different from other approaches such as Wigner-Weyl-Moyal scheme, quantum-mechanics-on-phase-space theory or coherent state approach. Finally, our construction of classical properties uses the way analogous to that of statistical physics. Thus, our MCP is a basis of modelling classical properties of quantum systems.

\section{$5.2 \quad$ A model of thermodynamic properties}

Here, we shall construct the length of a body as an average value with a small variance. No original calculation is to be expected, but simple and well known ideas are carefully interpreted. This entails that, first, the quantum structure of the system must be defined, second, the structural properties such as the energy spectrum calculated, third, some assumptions on the state of the system done and fourth, some dynamical properties derived that satisfy our definition of classical property.

\subsubsection{Composition, Hamiltonian and spectrum}

We shall consider a linear chain of $N$ identical particles of mass $\mu$ distributed along the $x$-axis with the quantum Hamiltonian

$$
\mathrm{H}=\frac{1}{2 \mu} \sum_{n=1}^{N} \mathrm{p}_{n}^{2}+\frac{\kappa^{2}}{2} \sum_{n=2}^{N}\left(\mathrm{x}_{n}-\mathrm{x}_{n-1}-\xi\right)^{2},
$$

involving only nearest-neighbour elastic forces. Here operator $\mathrm{x}_{n}$ is the position, operator $\mathrm{p}_{n}$ the momentum of the $n$-th particle, $\kappa$ the oscillator strength and $\xi$ the equilibrium interparticle distance. The parameters $\mu, \kappa$ and $\xi$ are structural properties (the last two defining the potential function).

This kind of chain seems to be different from most that are studied in literature: the positions of the chain particles are dynamical variables so that the chain can move as a whole. However, the chain can still be solved by methods that are described in [39, 64].

First, we find the variables $\mathbf{u}_{n}$ and $\mathrm{q}_{n}$ that diagonalize the Hamiltonian and define 
normal modes. The transformation is

$$
\mathrm{x}_{n}=\sum_{m=0}^{N-1} Y_{n}^{m} \mathbf{u}_{m}+\left(n-\frac{N+1}{2}\right) \xi,
$$

and

$$
\mathrm{p}_{n}=\sum_{m=0}^{N-1} Y_{n}^{m} \mathbf{q}_{m}
$$

where the mode index $m$ runs through $0,1, \cdots, N-1$ and $Y_{n}^{m}$ is an orthogonal matrix; for even $m$,

$$
Y_{n}^{m}=A(m) \cos \left[\frac{\pi m}{N}\left(n-\frac{N+1}{2}\right)\right]
$$

while for odd $m$,

$$
Y_{n}^{m}=A(m) \sin \left[\frac{\pi m}{N}\left(n-\frac{N+1}{2}\right)\right]
$$

and the normalization factors are given by

$$
A(0)=\frac{1}{\sqrt{N}}, \quad A(m)=\sqrt{\frac{2}{N}}, \quad m>0 .
$$

To show that $\mathbf{u}_{n}$ and $\mathrm{q}_{n}$ do represent normal modes, we substitute Eqs. (5.4) and (5.5) into (5.3) and obtain, after some calculation,

$$
\mathrm{H}=\frac{1}{2 \mu} \sum_{m=0}^{N-1} \mathbf{q}_{m}^{2}+\frac{\mu}{2} \sum_{m=0}^{N-1} \omega_{m}^{2} \mathbf{u}_{m}^{2},
$$

which is indeed diagonal. The mode frequencies are

$$
\omega_{m}=\frac{2 \kappa}{\sqrt{\mu}} \sin \frac{m}{N} \frac{\pi}{2} .
$$

Consider the terms with $m=0$. We have $\omega_{0}=0$, and $Y_{n}^{0}=1 / \sqrt{N}$. Hence,

$$
\mathrm{u}_{0}=\sum_{n=1}^{N} \frac{1}{\sqrt{N}} \mathrm{x}_{n}, \quad \mathrm{q}_{0}=\sum_{n=1}^{N} \frac{1}{\sqrt{N}} \mathrm{p}_{n},
$$

so that

$$
\mathrm{u}_{0}=\sqrt{N} \mathrm{X}, \quad \mathrm{q}_{0}=\frac{1}{\sqrt{N}} \mathrm{P},
$$

where $\mathrm{X}$ is the centre-of-mass coordinate of the chain and $\mathrm{P}$ is its total momentum. The 'zero' terms in the Hamiltonian then reduce to

$$
\frac{1}{2 M} \mathrm{P}^{2}
$$


with $M=N \mu$ being the total mass. Thus, the 'zero mode' describes a straight, uniform motion of the chain as a whole. The other modes are harmonic oscillators called 'phonons' with eigenfrequencies $\omega_{m}, m=1,2, \ldots, N-1$. The energy spectrum of our system is built from the mode frequencies by the formula

$$
E=\sum_{m=1}^{N-1} \nu_{m} \hbar \omega_{m},
$$

where $\left\{\nu_{m}\right\}$ is an $(N-1)$-tuple of non-negative integers-phonon occupation numbers.

At this stage, a new and important assumption must be done. We imagine that all states $\mathrm{T}$ of the modes $m=1, \cdots, N-1$ are prepared that have the same average internal energy $E$,

$$
\operatorname{Tr}\left[\mathrm{T}\left(H-\frac{P^{2}}{2 M}\right)\right]=E .
$$

We further assume that it is done in a perfectly random way, i.e., all other conditions or bias are to be excluded. Hence, the resulting state must maximize the entropy. In this way, the maximum of entropy does not represent an additional condition but rather the absence of any, see Sec. 3.1. The resulting state $T_{E}$ is the Gibbs state of the internal degrees of freedom. The conditions that define the preparation of Gibbs state are objective and need not be connected with human laboratory activity.

The internal energy has itself a very small relative variance in the Gibbs state; this need not be assumed from the start. Thus, it is a classical property. All other classical internal properties will turn out to be functions of the classical internal energy. Hence, for the internal degrees of freedom, $E$ forms itself a complete set of state coordinates introduced in Assumption 4.

The mathematics associated with the maximum entropy principle is variational calculus. The condition of fixed average energy is included with the help of Lagrange multiplier denoted by $\lambda$. It becomes a function $\lambda(E)$ for the resulting state. As it is well known, $\lambda(E)$ has to do with temperature.

The phonons of one species are excitation levels of a harmonic oscillator, so we have

$$
\mathrm{u}_{m}=\sqrt{\frac{\hbar}{2 \mu \omega_{m}}}\left(\mathrm{a}_{m}+\mathrm{a}_{m}^{\dagger}\right),
$$

where $\mathrm{a}_{m}$ is the annihilation operator for the $m$-th species. The diagonal matrix elements between the energy eigenstates $\left|\nu_{m}\right\rangle$ that we shall need then are

$$
\left\langle\nu_{m}\left|\mathbf{u}_{m}\right| \nu_{m}\right\rangle=0, \quad\left\langle\nu_{m}\left|\mathbf{u}_{m}^{2}\right| \nu_{m}\right\rangle=\frac{\hbar}{2 \mu \omega_{m}}\left(2 \nu_{m}+1\right) .
$$

For our system, the phonons of each species form statistically independent subsystems, hence the average of an operator concerning only one species in the Gibbs 
state $T_{\bar{E}}$ of the total system equals the average in the Gibbs state for the one species. Such a Gibbs state operator for the $m$-th species has the form

$$
\mathrm{T}_{m}=\sum_{\nu_{m}=0}^{\infty}\left|\nu_{m}\right\rangle p_{\nu_{m}}^{(m)}\left\langle\nu_{m}\right|
$$

where

$$
p_{\nu_{m}}^{(m)}=Z_{m}^{-1} \exp \left(-\hbar \lambda \omega_{m} \nu_{m}\right)
$$

and $Z_{m}$ is the partition function for the $m$-th species

$$
Z_{m}(\lambda)=\sum_{\nu_{m}=0}^{\infty} e^{-\lambda \hbar \omega_{m} \nu_{m}}=\frac{1}{1-e^{-\lambda \hbar \omega_{m}}}
$$

\subsubsection{The length of the body}

The classical property that will be defined and calculated in our quantum model is the average length of the body. Let us define the length operator by

$$
\mathrm{L}=\mathrm{x}_{N}-\mathrm{x}_{1}
$$

It can be expressed in terms of modes $\mathrm{u}_{m}$ using Eq. (5.4),

$$
\mathrm{L}=(N-1) \xi+\sum_{m=0}^{N-1}\left(Y_{N}^{m}-Y_{1}^{m}\right) \mathbf{u}_{m}
$$

The differences on the right-hand side are non-zero only for odd values of $m$, and equal then to $-2 Y_{1}^{m}$. We easily find, using Eqs. (5.7) and (5.8):

$$
\mathrm{L}=(N-1) \xi-\sqrt{\frac{8}{N}} \sum_{m=1}^{[N / 2]}(-1)^{m} \cos \left(\frac{2 m-1}{N} \frac{\pi}{2}\right) \mathrm{u}_{2 m-1}
$$

The average length is obtained using Eq. (5.11),

$$
\langle\mathrm{L}\rangle_{E}=(N-1) \xi
$$

It is a function of objective properties $N, \xi$ and $E$.

Eq. (5.14) is an important result. It shows that contributions to the length are more or less evenly distributed over all odd modes. Such a distribution leads to a very small variance of $L$ in Gibbs states. A lengthy calculation [29] gives for large $N$

$$
\frac{\Delta \mathrm{L}}{\langle\mathrm{L}\rangle_{E}} \approx \frac{2 \sqrt{3}}{\pi \kappa \xi \sqrt{\lambda}} \frac{1}{\sqrt{N}}
$$


Thus, the small relative variance for large $N$ need not be assumed from the start. The only assumptions are values of some structural properties and that an average value of energy is fixed. In the sense explained in Section 5.1, the length is then a classical property of our model body. We have obtained even more information, viz. the internal-energy dependence of the length (in this model, the dependence is trivial). This is an objective relation that can be in principle tested by measurements.

Similar results can be obtained for further thermodynamic properties such as elasticity coefficient, specific heat etc. They all are well known to have small variances in Gibbs states. The reason is that the contributions to these quantities are evenly distributed over the normal modes and the modes are mechanically and statistically independent. Further important classical properties are the mechanical ones: centre of mass and total momentum. In fact, these quantities can be chosen as the remaining state coordinates for the whole chain. The contributions to them are evenly distributed over all atoms, not modes: the bulk motion is mechanically and statistically independent of all other modes and so its variances will not be small in Gibbs states. Still, generalized statistical methods can be applied to it. This is done in the next section.

The last remark is that the thermodynamic equilibrium can settle down starting from an arbitrary state only if some weak but non-zero interaction exists between the phonons. We assume that this can be arranged so that the influence of the interaction on our result is negligible.

\subsection{Maximum entropy assumption in classical mechanics}

Let us start with the warning that the subject of this section has nothing to do with what is usually called 'statistical mechanics'.

If one is going to model classical mechanics then what are the properties that one would like to reproduce? The most conspicuous property from the point of view of quantum mechanics appears to be the sharpness of mechanical trajectories in the phase space because quantum mechanics denies the existence of such trajectories. This leads most researchers to aim at quantum states the phase-space picture of which is as sharp as possible. That are states with minimum uncertainty allowed by quantum mechanics. For one degree of freedom, described by coordinate $q$ and momentum $\mathrm{p}$, the uncertainty is given by the quantity

$$
\nu=\frac{2 \Delta q \Delta p}{\hbar}
$$

where $\Delta$ a denotes the variance of quantity a, as defined by Eq. (2.3). 
The states with $\nu=1$ are, however, very special states. First, they must be pure states such as Gaussian wave packets or coherent states. Such states are very difficult to prepare unlike the usual states of macroscopic systems described by classical mechanics. They are also prone to strong distortion by measurements. Moreover, as pure states, they can be linearly superposed. This is another peculiarity that is never observed for states of systems of classical mechanics. Hence, trying to get a trajectory as sharp as possible entails the loss of other desirable properties.

Moreover, the systems to which classical mechanics is applicable, are macroscopic. The overwhelming number of their degrees of freedom decouple from their mechanical ones, of which there are always only very few. The purely mechanical degrees of freedom have to do, e.g., with the motion of the centre of mass of a small but macroscopic body. As explained at the beginning of this chapter, the sharpness of phase-space trajectories is just an idealisation, a limit in which things become mathematically simpler. We can use it in calculations which, however, must also take into account the necessary non-zero variances of real observations. Indeed, such observations are generally afflicted with uncertainties $\nu \gg 1$. Hence, if we want to compare the predictions of quantum models with observations of classical mechanics, we are forced to compare states that are fuzzy in both theories.

Our main idea is to consider states with given averages and variances of coordinates and momenta, $Q^{k}, \Delta Q^{k}, P_{k}, \Delta P_{k}$, and leave everything else as fuzzy as possible. Thus, the averages of coordinates, of their squares, of momenta, and of their squares are fixed. To calculate the corresponding probability distributions in classical, and the state operators in quantum mechanics, we shall, therefore, apply the maximum entropy principle. The resulting states are called maximum-entropy packets, ME packets. The averages of coordinates and momenta take over the role of coordinate and momenta in classical mechanics. In any case these averages represent measurable aspects of these variables. The dynamical evolution of variances is an important indicator of the applicability of the model one is working with. It determines the time intervals within which reasonable predictions are possible.

Consider a three-body system that is to model the Sun, Earth and Jupiter in Newton mechanics. It turns out that generic trajectories starting as near to each other as, say, the dimension of the irregularities of the Earth surface will diverge from each other by dimensions of the Earth-Sun distance after the time of only about $10^{7}$ years. This seems to contradict the $4 \times 10^{12}$ years of relatively stable Earth motion around the Sun that is born out by observations. The only way out is the existence of a few special trajectories that are much stabler than the generic ones and the fact that bodies following an unstable trajectory have long ago fallen into the Sun or have been ejected from the solar system. This spontaneous evolution can be considered as a preparation procedure of solar system. 
As explained in Sec. 1.1.2, a prepared state can be viewed as objective. A simple example is a gun in a position that is fixed in a reproducible way and that shoots bullets using cartridges of a given provenance. The state of each individual shot is defined by the conditions and is the same for all shots even if observations may have different results for different shots. In the theoretical description of a state, we can make the limit of $\Delta Q^{k} \rightarrow 0, \Delta P_{k} \rightarrow 0$. This is considered as a non-existing, but practically useful idealization.

A finer analysis is possible only as long as new preparations are specified that determine a subset of individual shots. For example, if the conditions on the gun are that the gun is held by a human hand, then one cause of the relatively large $\Delta Q^{k}$ and $\Delta P_{k}$ is that the gun is always in a slightly different position because the hand and eye are not completely sure. The cause is 'classical' in its nature and one can imagine that each shot will be repeated several times more under the condition of a mechanical fixation. Then, there will still be variances but they may be much smaller. Thus, the original preparation can be considered as composed of sub-preparations in the way of gemenge.

In general, if a part of the variances in a state come about as random or controlled changes of classical conditions in the preparation procedure, then the preparation is clearly composed of sub-preparations and the state a gemenge. Some variances will still remain, but they will be 'sufficiently' small for the corresponding states to be viewed as defined by the corresponding averages and negligible variances.

To limit ourselves just to given averages and variances of coordinates and momenta is a great simplification that enables us to obtain interesting results easily.

\subsection{Classical ME packets}

Let us first consider a system $\mathcal{S}$ with one degree of freedom (the generalization to any number is easy). Let the coordinate be $q$ and the momentum $p$. A state is a distribution function $\rho(q, p)$ on the phase space spanned by $q$ and $p$. The function $\rho(q, p)$ is dimensionless and normalized by

$$
\int \frac{d q d p}{v} \rho=1
$$

where $v$ is an auxiliary phase-space volume to make the integration dimensionless. The entropy of $\rho(q, p)$ can be defined by

$$
S:=-\int \frac{d q d p}{v} \rho \ln \rho .
$$

The value of entropy will depend on $v$ but most other results will not. Classical mechanics does not offer any idea of how to fix $v$. We shall get its value from quantum mechanics. 


\subsubsection{Definition and properties}

Definition 21 ME packet is the distribution function $\rho$ that maximizes the entropy subject to the conditions:

$$
\langle q\rangle=Q, \quad\left\langle q^{2}\right\rangle=\Delta Q^{2}+Q^{2},
$$

and

$$
\langle p\rangle=P, \quad\left\langle p^{2}\right\rangle=\Delta P^{2}+P^{2},
$$

where $Q, P, \Delta Q$ and $\Delta P$ are given values.

We have used the abbreviation

$$
\langle x\rangle=\int \frac{d q d p}{v} x \rho .
$$

The explicit form of $\rho$ can be found using the partition-function method [30]. The resulting partition function for classical ME packets is

$$
Z=\frac{\pi}{v} \frac{1}{\sqrt{\lambda_{3} \lambda_{4}}} \exp \left(\frac{\lambda_{1}^{2}}{4 \lambda_{3}}+\frac{\lambda_{2}^{2}}{4 \lambda_{4}}\right) .
$$

The four Lagrange multipliers $\lambda_{1}, \lambda_{3}, \lambda_{2}$ and $\lambda_{4}$ correspond to the four conditions (5.18) and (5.19)

The generalization to any number of dimensions is trivial.

Proposition 19 The distribution function of the ME packet for a system with given averages and variances $Q_{1}, \cdots, Q_{n}, \Delta Q_{1}, \cdots, \Delta Q_{n}$ of coordinates and $P_{1}, \cdots, P_{n}$, $\Delta P_{1}, \cdots, \Delta P_{n}$ of momenta, is

$$
\rho=\left(\frac{v}{2 \pi}\right)^{n} \prod_{k=1}^{n}\left(\frac{1}{\Delta Q_{k} \Delta P_{k}} \exp \left[-\frac{\left(q_{k}-Q_{k}\right)^{2}}{2 \Delta Q_{k}^{2}}-\frac{\left(p_{k}-P_{k}\right)^{2}}{2 \Delta P_{k}^{2}}\right]\right) .
$$

For proof, see Ref. [30]. We observe that all averages obtained from $\rho$ are independent of $v$ and that the result is a Gaussian distribution in agreement with Jaynes' conjecture that the maximum entropy principle gives the Gaussian distribution if the only conditions are fixed values of the first two moments.

As $\Delta Q$ and $\Delta P$ approach zero, $\rho$ becomes a delta-function and the state becomes sharp. For some quantities this limit is sensible for others it is not. In particular, the entropy, which can easily be calculated,

$$
S=1+\ln \frac{2 \pi \Delta Q \Delta P}{v},
$$

diverges to $-\infty$. This is due to a general difficulty in giving a definition of entropy for a continuous system that would be satisfactory in every respect. What one could do is to divide the phase space into cells of volume $v$ so that $\Delta Q \Delta P$ could not be chosen smaller than $v$. Then, the limit $\Delta Q \Delta P \rightarrow v$ of entropy would make more sense. 


\subsubsection{Classical equations of motion}

Let us assume that the Hamiltonian of $\mathcal{S}$ has the form

$$
H=\frac{p^{2}}{2 \mu}+V(q)
$$

where $\mu$ is the mass and $V(q)$ the potential function. The equations of motion are

$$
\dot{q}=\{q, H\}, \quad \dot{p}=\{p, H\}
$$

Inserting (5.22) for $H$, we obtain

$$
\dot{q}=\frac{p}{\mu}, \quad \dot{p}=-\frac{d V}{d q}
$$

The general solution to these equations can be written in the form

$$
q(t)=q(t ; q, p), \quad p(t)=p(t ; q, p)
$$

where

$$
q(0 ; q, p)=q, \quad p(0 ; q, p)=p,
$$

$q$ and $p$ being arbitrary initial values. We obtain the equations of motion for the averages and variances if the initial state is an ME packet:

$$
Q(t)=\langle q(t ; q, p)\rangle, \quad \Delta Q(t)=\sqrt{\left\langle(q(t ; q, p)-Q(t))^{2}\right\rangle}
$$

and

$$
\left.P(t)=\langle p(t ; q, p)\rangle, \quad \Delta P(t)=\sqrt{\left\langle(p(t ; q, p)-P(t))^{2}\right.}\right\rangle .
$$

In general, $Q(t)$ and $P(t)$ will depend not only on $Q$ and $P$, but also on $\Delta Q$ and $\Delta P$.

Let us consider the special case of at most quadratic potential:

$$
V(q)=V_{0}+V_{1} q+\frac{1}{2} V_{2} q^{2}
$$

where $V_{k}$ are constants with suitable dimensions. If $V_{1}=V_{2}=0$, we have a free particle, if $V_{2}=0$, it is a particle in a homogeneous force field and if $V_{2} \neq 0$, it is an harmonic or anti-harmonic oscillator.

In this case, the general solution has the form

$$
\begin{aligned}
& q(t)=f_{0}(t)+q f_{1}(t)+p f_{2}(t), \\
& p(t)=g_{0}(t)+q g_{1}(t)+p g_{2}(t),
\end{aligned}
$$


where $f_{0}(0)=f_{2}(0)=g_{0}(0)=g_{1}(0)=0$ and $f_{1}(0)=g_{2}(0)=1$. If $V_{2} \neq 0$, the functions are

$$
\begin{array}{r}
f_{0}(t)=-\frac{V_{1}}{V_{2}}(1-\cos \omega t), \quad f_{1}(t)=\cos \omega t, \quad f_{2}(t)=\frac{1}{\xi} \sin \omega t \\
g_{0}(t)=-\xi \frac{V_{1}}{V_{2}} \sin \omega t, \quad g_{1}(t)=-\xi \sin \omega t, \quad g_{2}(t)=\cos \omega t
\end{array}
$$

where

$$
\xi=\sqrt{\mu V_{2}}, \quad \omega=\sqrt{\frac{V_{2}}{\mu}} .
$$

Only for $V_{2}>0$, the functions remain bounded. If $V_{2}=0$, we obtain

$$
\begin{array}{ccc}
f_{0}(t)=-\frac{V_{1}}{2 \mu} t^{2}, & f_{1}(t)=1, & f_{2}(t)=\frac{t}{\mu} \\
g_{0}(t)=-V_{1} t, & g_{1}(t)=0, & g_{2}(t)=1 .
\end{array}
$$

The equations for averages and variances resulting from Eqs. (5.24), (5.18) and (5.19) are [30]

$$
\begin{aligned}
Q(t) & =f_{0}(t)+Q f_{1}(t)+P f_{2}(t), \\
\Delta Q(t) & =\sqrt{f_{1}^{2}(t) \Delta Q^{2}+f_{2}^{2}(t) \Delta P^{2}}, \\
P(t) & =g_{0}(t)+Q g_{1}(t)+P g_{2}(t), \\
\Delta P(t) & =\sqrt{f_{g}^{2}(t) \Delta Q^{2}+g_{2}^{2}(t) \Delta P^{2}}
\end{aligned}
$$

We observe: if functions $f_{1}(t), f_{2}(t), g_{1}(t)$ and $g_{2}(t)$ remain bounded, the variances also remain bounded and the predictions are possible in arbitrary long intervals of time. Otherwise, there will always be only limited time intervals in which the theory can make predictions.

In the case of general potential, the functions (5.24) can be expanded in products of powers of $q, p$ and $t$, and the averages of these products can be calculated by partition function method. In Ref. [30], equations of motion are calculated up to fourth order.

\subsection{Quantum ME packets}

Let us now turn to quantum mechanics and try to solve an analogous problem. 


\subsubsection{Definition and properties}

Definition 22 Let the quantum model $\mathcal{S}_{q}$ of system $\mathcal{S}$ has the basic observables $\mathrm{q}$ and p. State T that maximizes von Neumann entropy (see Sec. 3.1)

$$
S=-\operatorname{tr}(\mathrm{T} \ln \mathrm{T})
$$

under the conditions

$$
\begin{aligned}
& \operatorname{tr}[\mathrm{Tq}]=Q, \quad \operatorname{tr}\left[\mathrm{Tq}^{2}\right]=Q^{2}+\Delta Q^{2}, \\
& \operatorname{tr}[\mathrm{T} \mathrm{p}]=P, \quad \operatorname{tr}\left[\mathrm{Tp}^{2}\right]=P^{2}+\Delta P^{2},
\end{aligned}
$$

where $Q, P, \Delta Q$ and $\Delta P$ are given numbers, is called quantum ME packet.

To calculate the state operator of a ME packet, one can use the partition function method. However, it must be modified because the involved quantities do not commute, see Ref. [30]. The partition function for the quantum ME packets is

$$
Z=\frac{\exp \left(\frac{\lambda_{1}^{2}}{4 \lambda_{3}}+\frac{\lambda_{2}^{2}}{4 \lambda_{4}}\right)}{2 \sinh \left(\hbar \sqrt{\lambda_{3} \lambda_{4}}\right)} .
$$

The resulting state operator, generalised to $n$ degrees of freedom is described by the following

Proposition 20 The state operator of the ME packet of a system with given averages and variances $Q_{1}, \cdots, Q_{n}, \Delta Q_{1}, \cdots, \Delta Q_{n}$ of coordinates and $P_{1}, \cdots, P_{n}$, $\Delta P_{1}, \cdots, \Delta P_{n}$ of momenta, is

$$
\mathrm{T}=\prod_{k=1}^{n}\left[\frac{2}{\nu_{k}^{2}-1} \exp \left(-\frac{1}{\hbar} \ln \frac{\nu_{k}+1}{\nu_{k}-1} \mathrm{~K}_{k}\right)\right],
$$

where

$$
\mathrm{K}_{k}=\frac{1}{2} \frac{\Delta P_{k}}{\Delta Q_{k}}\left(\mathrm{q}_{k}-Q_{k}\right)^{2}+\frac{1}{2} \frac{\Delta Q_{k}}{\Delta P_{k}}\left(\mathrm{p}_{k}-P_{k}\right)^{2}
$$

and

$$
\nu_{k}=\frac{2 \Delta P_{k} \Delta Q_{k}}{\hbar}
$$

For the proof, see Ref. [30]. The calculation of the state operator is simplified by the fact that $\mathrm{K}_{k}$ is Hamiltonian operator of a harmonic oscillator. Of course, system $\mathcal{S}_{q}$ for which the calculation is done, is completely general and its Hamiltonian operator has nothing to do with $\mathrm{K}_{k}$.

Strictly speaking, the state operator (5.43) is not a Gaussian distribution. Thus, it seems to be either a counterexample to, or a generalization of, Jaynes' hypothesis. 
Everything can be decomposed into well-known eigenstates $|m\rangle$ of $\mathbf{K}$. We have $(n=1)$

$$
\mathrm{K}=\sum_{k=0}^{\infty} R_{m}|m\rangle\langle m|
$$

We easily obtain for $R_{m}$ that

$$
R_{m}=2 \frac{(\nu-1)^{m}}{(\nu+1)^{m+1}}
$$

Hence,

$$
\lim _{\nu \rightarrow 1} R_{m}=\delta_{m 0},
$$

and the state $\mathrm{T}$ becomes $|0\rangle\langle 0|$. In general, states $|m\rangle$ depend on $\nu$. The state vector $|0\rangle$ expressed as a function of $Q, P, \Delta Q$ and $\nu$ is given by

$$
\psi(q)=\left(\frac{1}{\pi} \frac{\nu}{2 \Delta Q^{2}}\right)^{1 / 4} \exp \left[-\frac{\nu}{4 \Delta Q^{2}}(q-Q)^{2}+\frac{i P q}{\hbar}\right] .
$$

This is a Gaussian wave packet that corresponds to other values of variances than the original ME packet but has the minimal uncertainty. For $\nu \rightarrow 1$, it remains regular and the projection $|0\rangle\langle 0|$ becomes the state operator of the original ME packet. Hence, Gaussian wave packets are special cases of quantum ME packets.

\subsubsection{Quantum equations of motion}

Let the Hamiltonian of $\mathcal{S}_{q}$ be $\mathrm{H}$ and the unitary evolution group be $\mathrm{U}(t)$. The dynamics in the Schrödinger picture leads to the time dependence of T:

$$
\mathbf{T}(t)=\mathbf{U}(t) \mathbf{T} \mathbf{U}(t)^{\dagger} .
$$

Substituting for T from Eq. (5.43) and using a well-known property of exponential functions, we obtain

$$
\mathbf{T}(t)=\frac{2}{\nu^{2}-1} \exp \left(-\frac{1}{\hbar} \ln \frac{\nu+1}{\nu-1} \mathrm{U}(t) \mathrm{KU}(t)^{\dagger}\right) .
$$

In the Heisenberg picture, $T$ remains constant, while $q$ and $p$ are time dependent and satisfy the equations

$$
i \hbar \frac{d \mathbf{q}}{d t}=[\mathbf{q}, \mathrm{H}], \quad i \hbar \frac{d \mathbf{p}}{d t}=[\mathrm{p}, \mathrm{H}] .
$$

They are solved by

$$
\mathbf{q}(t)=\mathbf{U}(t)^{\dagger} \mathbf{q} \mathbf{U}(t), \quad \mathrm{p}(t)=\mathbf{U}(t)^{\dagger} \mathrm{p} \mathbf{U}(t),
$$


where $\mathrm{q}$ and $\mathrm{p}$ are the initial operators, $\mathrm{q}=\mathrm{q}(0)$ and $\mathrm{p}=\mathrm{p}(0)$. The resulting operators can be written in the form of operator functions analogous to classical expressions (5.24) so that Eqs. (5.26) and (5.27) can again be used.

The example with potential function (5.28) is solvable in quantum theory, too, and we can use it for comparison with the classical dynamics as well as for a better understanding of the ME packet dynamics. Eqs. (5.50) have then the solutions given by (5.29) and (5.30) with functions $f_{n}(t)$ and $g_{n}(t)$ given by (5.31) and (5.32) or (5.33) and (5.34). The calculation of the averages and variances is analogous to the classical one and we obtain Eqs. (5.35) and Eq. (5.36) again. Similarly for p, the results are given by Eqs. (5.37) and (5.38).

We have shown that the averages and variances of quantum ME packets have exactly the same time evolution as those of classical ME packets in the special case of at-most-quadratic potentials. From formulae (5.36) and (5.38) we can also see an interesting fact. On the one hand, both variances must increase near $t=0$. On the other, the entropy must stay constant because the evolution of the quantum state is unitary. As the relation between entropy and $\nu$ is fixed for ME packets, the ME packet form is not preserved by the evolution (the entropy ceases to be maximal). This is similar for Gaussian-packet form or for coherent-state form.

For general potentials, there will be two types of corrections to the dynamics of the averages: terms containing the variances and terms containing $\hbar$. To see these corrections, equations of motion have been expanded in powers of $\mathrm{q}, \mathrm{p}$ and $t$ in Ref. [30]. The conclusion has been that the quantum equations begin to differ from the classical ones only in the higher order terms in $V$ or in the higher time derivatives and the correction is of the second order in $1 / \nu$. This seems to be very satisfactory: our quantum model reproduces the classical dynamic very well. Moreover, Eq. (5.48) shows that Gaussian wave packets are special cases of ME packets with $\nu=1$. Thus, they approximate classical trajectories less accurately than ME packets with large $\nu$.

\subsection{Classical limit}

Let us now look to see if our equations give some support to the statement that $\nu \gg 1$ is the classical regime.

The quantum partition function (5.42) differs from its classical counterpart (5.20) by the denominator $\sinh \left(\hbar \sqrt{\lambda_{3} \lambda_{4}}\right)$. If

$$
\hbar \sqrt{\lambda_{3} \lambda_{4}} \ll 1
$$

we can write

$$
\sinh \left(\hbar \sqrt{\lambda_{3} \lambda_{4}}\right)=\hbar \sqrt{\lambda_{3} \lambda_{4}}\left[1+O\left(\left(\hbar \sqrt{\lambda_{3} \lambda_{4}}\right)^{2}\right)\right]
$$


The leading term in the partition function then is

$$
Z=\frac{\pi}{h} \frac{1}{\sqrt{\lambda_{3} \lambda_{4}}} \exp \left(\frac{\lambda_{1}^{2}}{4 \lambda_{3}}+\frac{\lambda_{2}^{2}}{4 \lambda_{4}}\right),
$$

where $h=2 \pi \hbar$. Comparing this with formula (5.20) shows that the two expressions are identical, if we set

$$
v=h .
$$

We can say that quantum mechanics gives us the value of $v$. Next, we have to express condition (5.51) in terms of the averages and variances. The usual relations between the Lagrange multipliers on the one hand and the averages and variances on the other (see Ref. [30]) imply

$$
\hbar \sqrt{\lambda_{3} \lambda_{4}}=\frac{1}{2} \ln \frac{\nu+1}{\nu-1} .
$$

Hence, condition (5.51) is equivalent to

$$
\nu \gg 1 .
$$

The result can be formulated as follows. Classical mechanics allows not only sharp, but also fuzzy trajectories and the comparison of some classical and quantum fuzzy trajectories shows a very good match. The fuzzy states chosen here are the so-called ME packets. Their fuzziness is described by the quantity $\nu=2 \Delta Q \Delta P / \hbar$. The entropy of an ME packet depends only on $\nu$ and is an increasing function of it. The time evolution of classical and quantum ME packets with the same initial values of averages and variances defines the averages as time functions. The larger $\nu$ is, the better the quantum and the classical evolutions of average values have been shown to agree. Thus, the classical regime is neither $\Delta Q=\Delta P=0$ (absolutely sharp trajectory) nor $\nu=1$ (minimum quantum uncertainty). This is the most important result of Ref. [30]. The time functions coincide for the two theories in the limit $\nu \rightarrow \infty$. Hence, in our approach, this is the classical limit. It is very different from the usual assumption that the classical limit must yield the variances as small as possible. Of course, $\nu$ can be very large and still compatible with classically negligible variances.

One also often requires that commutators of observables vanish in classical limit. This is however only motivated by the assumption that all basic quantum properties are single values of observables. Within our interpretation, this assumption is rejected and if classical observables are related to quantum operators then only by being average values of the operators in prepared states. Then, first, all such averages are defined by a preparation and do exist simultaneously, independently of whether the operators commute or not. For example, $Q$ and $P$ are such simultaneously existing variables for ME packets. Second, a joint measurement of fuzzy 
values of non-commuting observables is possible. In Sec. 4.4, we have shown Eq. (4.9), which is a general formula valid for an arbitrary state $T_{\mathcal{A}}$ of the ancilla. Then, we have chosen $\mathrm{T}_{\mathcal{A}}$ to be a Gaussian wave packet, which has $\nu=1$. However, $\mathrm{T}_{\mathcal{A}}$ can also be chosen as a quantum ME packet with $Q=0, P=0$ and arbitrary $\Delta Q$ and $\Delta P$ allowing arbitrary large $\nu$. The resulting observable represents the detection of a shifted and boosted ME packet.

Let us compare the present paper notion of classical limit with a modern textbook version such as Chap. 14 of [3]. Both approaches define the classical limit of a quantum state as a classical ensemble described by a fuzzy distribution function and calculate time evolutions of averages in the states. However, in the textbook, any quantum system, even not macroscopic, and any state, even pure, are allowed (pure states are preferred as they have smaller uncertainties). Hence, our notion is much narrower: we consider only macroscopic quantum systems and only some of their maximum entropy states. This has obvious physical reasons explained in Secs. 5.1 and 5.3 . 


\section{Chapter 6}

\section{Quantum models of preparation and registration of microsystems}

Discussions about the nature of quantum measurement were started already by founding fathers of quantum theory, persisted throughout and seem even to amplify at the present time.

An old approach to the problem of quantum measurement is Bohr's (its newer, rigorously reformulated version is Ref. [48]). This approach denies that measuring apparatuses, and all classical systems in general, are quantum systems in the sense that all their properties can be derived from, or are compatible with, quantum mechanics. They must be described by other theories, called pretheories. Of course some classical properties of macroscopic systems can be obtained by quantum statistics. Ref. [49] shows that such occasional applications of quantum mechanics to classical systems are compatible with the form of denying the universality of quantum mechanics specified there.

Modern approaches assume the universality of quantum mechanics together with various further ideas. A very brief account of the three most important examples is:

1. Quantum decoherence theory [75, 76]. The idea is that system $\mathcal{S}+\mathcal{A}$ composite of a quantum object and an apparatus cannot be isolated from environment $\mathcal{E}$. Then the unitary evolution of $\mathcal{S}+\mathcal{A}+\mathcal{E}$ leads to a non-unitary evolution of $\mathcal{S}+\mathcal{A}$ that can erase all correlations and interferences from $\mathcal{S}+\mathcal{A}$ that hinder the objectification (see the Introduction). However, then the correlations and interferences are just transferred to $\mathcal{S}+\mathcal{A}+\mathcal{E}$ and the method can only work, if some bizarre assumptions are made about world, for instance one accepts some variant of the many world interpretation.

2. Superselection sectors approach [34, 62]. Here, classical properties are described by superselection observables of $\mathcal{A}$ which commute with each other 
and with all other observables of it (see Sec. 3.3). Then, the state of $\mathcal{A}$ after the measurement is equivalent to that with a suitable gemenge structure. The problems are that, first, the Hamiltonian of the measurement coupling cannot be an observable or else the measurement model does not work. Second, the only known physical mechanism for producing strict superselection rules occurs in systems having infinitely many degrees of freedom. However, measuring apparatuses do not satisfy this conditions and the superselection rules can therefore be only approximative.

3. Modal interpretation [15]. One assumes that there is a subset of projections that have determinate values in the state (of $\mathcal{S}+\mathcal{A}$ ) after the registration. The set of projections must just be such that it does not violate contextuality (see Sec. 3.2.2), even if it can violate the existence of correlations and interferences. Thus, one must postulate that the measurements of the correlations and interferences on the state are forbidden for some unknown reason.

In general, the problem is far from being satisfactorily solved by any of the modern theories. Analysis of Refs. [22, 15, 16], as well as of our previous papers [29, 30], give an account of their shortcomings. We adopt the definition of the problem and the proof that it is far from being solved from Ref. [16], Chap. IV, where the above short points are dealt with in more detail and the corresponding references are given.

Our approach to the problem is very different from anything that has ever been published in the field. It is based on a physical idea rather than on some spectacular mathematics. It seems to promise a complete solution to the problem. To explain the idea, we make some simplifying assumptions. First, we restrict ourselves to measurements performed on microsystems, i.e. particles and systems composite of few particles. There are other systems on which recently a lot of interesting experiments has been done, such as Bose-Einstein condensates, strong laser beams or currents in superconducting rings. Such quantum states of 'large' systems, mezoscopic or even macroscopic, will be ignored here. Second, we use a simplified model of measurement.

\subsection{Beltrametti-Cassinelli-Lahti model}

In this section, we are going to recapitulate the well-known ideas on measurement that will be needed later. A summary is [16], p. 25:

... the object system $\mathcal{S}$, prepared in a state $\mathrm{T}$ is brought into a suitable contact - a measurement coupling - with another, independently prepared system, the measurement apparatus from which the result related to the 
measured observable $\mathrm{O}$ is determined by reading the value of the pointer observable.

In Ref. [16], these ideas are developed in detail with the help of models. One of them, which we call Beltrametti-Cassinelli-Lahti (BCL) model (p. 38), is as follows. Let a discrete observable $\mathrm{O}$ of system $\mathcal{S}$ of type $\tau$ with Hilbert space $\mathbf{H}_{\tau}$ be measured. Let $o_{k}$ be eigenvalues and $\left\{\phi_{k j}\right\}$ be the complete orthonormal set of eigenvectors of $\mathrm{O}$

$$
\mathrm{O} \phi_{k j}=o_{k} \phi_{k j}
$$

We assume that $k=1, \cdots, N$ so that there is only a finite number of different eigenvalues $o_{k}$. This is justified by the fact that no real registration apparatus can distinguish all elements of an infinite set from each other. It can therefore measure only a function of an observable that maps its spectrum onto a finite set of real numbers. Our observable $\mathrm{O}$ is such a function. The projection $\mathrm{E}_{k}^{\mathrm{O}}$ on the eigenspace of $o_{k}$ is then $\mathrm{E}_{k}^{\mathrm{O}}=\sum_{j}\left|\phi_{k j}\right\rangle\left\langle\phi_{k j}\right|$.

Let the registration apparatus 1 be a quantum system $\mathcal{A}$ with Hilbert space $\mathbf{H}_{\mathcal{A}}$ and an observable $\mathrm{A}$. Let $\mathrm{A}$ be a non-degenerate, discrete observable with the same eigenvalues $o_{k}$ and with orthonormal set of eigenvectors $\psi_{k}$,

$$
\mathrm{A} \psi_{k}=o_{k} \psi_{k}
$$

with possible further eigenstates (such as $\psi$ of Prop. 21) and eigenvalues. The projection on an eigenspace is $\mathrm{E}_{k}^{\mathrm{A}}=\left|\psi_{k}\right\rangle\left\langle\psi_{k}\right|$. $\mathrm{A}$ is the so-called pointer observable.

Let the measurement start with the preparation of $\mathcal{S}$ in state $\mathrm{T}$ and the independent preparation of $\mathcal{A}$ in state $\mathrm{T}_{\mathcal{A}}$. The initial state of the composed system $\mathcal{S}+\mathcal{A}$ is thus $\mathrm{T} \otimes \mathrm{T}_{\mathcal{A}}$.

Let $\mathcal{S}$ and $\mathcal{A}$ then interact for a finite time by the so-called measurement coupling and let the resulting state be given by $\mathrm{U}\left(\mathrm{T} \otimes \mathrm{T}_{\mathcal{A}}\right) \mathrm{U}^{\dagger}$, where $\mathrm{U}$ is a unitary transformation on $\mathbf{H}_{\tau} \otimes \mathbf{H}_{\mathcal{A}}$.

The final state of the apparatus is $\Pi_{\mathcal{S}}\left[\mathrm{U}\left(\mathrm{T} \otimes \mathrm{T}_{\mathcal{A}}\right) \mathrm{U}^{\dagger}\right]$, where $\Pi_{\mathcal{S}}$ is the partial trace over states of $\mathcal{S}$. The first requirement on the model is that this state gives the same probability measure for the pointer observable as the initial state $T$ predicted for the observable $\mathrm{O}$ :

$$
\operatorname{tr}\left[\mathrm{TE}_{k}^{\mathrm{O}}\right]=\operatorname{tr}\left[\Pi_{\mathcal{S}}\left[\mathrm{U}\left(\mathrm{T} \otimes \mathrm{T}_{\mathcal{A}}\right) \mathrm{U}^{\dagger}\right] \mathrm{E}_{k}^{\mathrm{A}}\right] .
$$

This is called probability reproducibility condition. Now, there is a theorem [8]:

Proposition 21 Let a measurement fulfil all assumptions and conditions listed above. Then, for any initial vector state $\psi$ of $\mathcal{A}$, there is a set $\left\{\varphi_{k l}\right\}$ of unit vectors

\footnotetext{
${ }^{1}$ In our language, a measurement consists of preparation and registration so that what Ref. [16] often calls 'measurement' is our 'registration'.
} 
in $\mathbf{H}_{\tau}$ satisfying the orthogonality conditions

$$
\left\langle\varphi_{k l} \mid \varphi_{k j}\right\rangle=\delta_{l j}
$$

such that $\mathrm{U}$ is a unitary extension of the map

$$
\phi_{k l} \otimes \psi \mapsto \varphi_{k l} \otimes \psi_{k}
$$

The second requirement on the model is that it has to lead to a definite result. More precisely, the apparatus must be in one of the states $\left|\psi_{k}\right\rangle\left\langle\psi_{k}\right|$ after each indi-

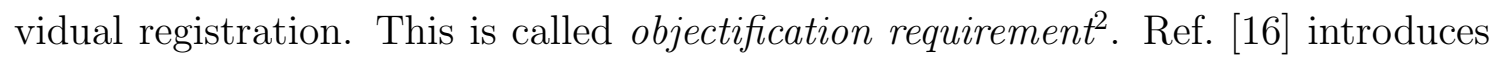
a more general concept of measurement that leaves open whether the objectification requirement is satisfied or not. Such a procedure is called premeasurement. A measurement is then a premeasurement that satisfies objectification requirement.

Suppose that the initial state of $\mathcal{S}$ is an eigenstate of $\mathrm{O}, \mathrm{T}=\left|\phi_{k l}\right\rangle\left\langle\phi_{k l}\right|$, with the eigenvalue $o_{k}$. Then, Eq. (6.2) implies that the final state of apparatus $\mathcal{A}$ is $\left|\psi_{k}\right\rangle\left\langle\psi_{k}\right|$, and the premeasurement does lead to a definite result. However, suppose next that the initial state is an arbitrary vector state, $\mathrm{T}=|\phi\rangle\langle\phi|$. Decomposing $\phi$ into the eigenstates,

$$
\phi=\sum_{k l} c_{k l} \phi_{k l}
$$

we obtain from Eq. (6.2)

$$
\Phi_{\mathrm{end}}=\mathrm{U}(\phi \otimes \psi)=\sum_{k} \sqrt{p_{k}} \varphi_{k}^{1} \otimes \psi_{k},
$$

where

$$
\varphi_{k}^{1}=\frac{\sum_{l} c_{k l} \varphi_{k l}}{\sqrt{\left\langle\sum_{l} c_{k l} \varphi_{k l} \mid \sum_{j} c_{k j} \varphi_{k j}\right\rangle}}
$$

and

$$
p_{k}=\left\langle\sum_{l} c_{k l} \varphi_{k l} \mid \sum_{j} c_{k j} \varphi_{k j}\right\rangle
$$

is the probability that a registration of $\mathrm{O}$ performed on vector state $\phi$ gives the value $o_{k}$. The final state of apparatus $\mathcal{A}$ then is

$$
t r_{\mathcal{S}}\left[\mathrm{U}\left(\mathrm{T} \otimes \mathrm{T}_{\mathcal{A}}\right) \mathrm{U}^{\dagger}\right]=\sum_{k l} \sqrt{p_{k}} \sqrt{p_{l}}\left\langle\varphi_{k}^{1} \mid \varphi_{l}^{1}\right\rangle\left|\psi_{k}\right\rangle\left\langle\psi_{l}\right|
$$

\footnotetext{
${ }^{2}$ Ref. [16] calls this pointer objectification and introduces a further requirement concerning the state of $\mathcal{S}$ after the registration, value objectification (pp. 30, 49). As our approach denies that objectivity of observable values is necessary for realist interpretation, the value objectification is not an important requirement for us, but it will follow from the pointer objectification via strong correlations between observable and pointer values valid in some models.
} 
Because of the orthonormality of $\left|\psi_{k}\right\rangle$ 's, the probability that the apparatus shows the value $o_{k}$ if $\mathrm{A}$ is registered on it in this final state is $p_{k}$, which is what the probability reproducibility requires. However, if the objectification requirement is to be satisfied, the final state of the apparatus must be

$$
t r_{\mathcal{S}}\left[\mathrm{U}\left(\mathrm{T} \otimes \mathrm{T}_{\mathcal{A}}\right) \mathrm{U}^{\dagger}\right]=\left(\sum_{j}\right) p_{j}\left|\psi_{j}\right\rangle\left\langle\psi_{j}\right|
$$

(it must be a gemenge, see Chapter 1, Sec. 1.2). Thus, in general we have a model of a premeasurement, but not of a measurement.

\subsubsection{Repeatable premeasurement and von Neumann model}

In order to define what a repeatable premeasurement is, we need the notion of state transformer. To this aim, let us first calculate the final state of system $\mathcal{S}$ after a BCL premeasurement is finished:

$$
\Pi_{\mathcal{A}}\left[\mathrm{U}(|\phi\rangle\langle\phi|\otimes| \psi\rangle\langle\psi|) \mathrm{U}^{\dagger}\right]=\sum_{k} p_{k}\left|\varphi_{k}^{1}\right\rangle\left\langle\varphi_{k}^{1}\right| .
$$

The part of the sum on the right-hand side corresponding to the result of premeasurement lying in the set $X$ is

$$
\mathcal{I}(X)(|\phi\rangle\langle\phi|)=\sum_{o_{k} \in X} p_{k}\left|\varphi_{k}^{1}\right\rangle\left\langle\varphi_{k}^{1}\right| .
$$

The right-hand side is not a state, because it is not normalised. Its trace is the probability that the result lies in $X$,

$$
p_{\mathrm{T}}^{\mathrm{O}}(X)=\operatorname{tr}[\mathcal{I}(X)(\mathrm{T})]
$$

if the initial state of $\mathcal{S}$ is $\mathrm{T}$. The quantity $\left(1 / p_{\mathrm{\top}}^{\mathrm{O}}(X)\right) \mathcal{I}(X)$ is an operation-valued 3 measure and is called state transformer. For more details, see Ref. [16].

Definition 23 A premeasurement is called repeatable if its state transformer satisfies the equation

$$
\operatorname{tr}[\mathcal{I}(Y)(\mathcal{I}(X)(\mathrm{T}))]=\operatorname{tr}[\mathcal{I}(Y \cap X)(\mathrm{T})]
$$

for all subsets of possible values $X$ and $Y$ and all possible states $\mathrm{T}$ of $\mathcal{S}$.

That is, the repetition of the premeasurement on $\mathcal{S}$ does not lead to any new result from the probabilistic point of view. To see whether the state transformer (6.7) satisfies Eq. (6.8), let us rewrite in the form:

$$
\mathcal{I}(X)(|\phi\rangle\langle\phi|)=\sum_{o_{k} \in X} p_{k}\left|\varphi_{k}^{1}\right\rangle\left\langle\varphi_{k}^{1}\left|=\sum_{o_{k} \in X} \mathrm{~K}_{k}\right| \phi\right\rangle\langle\phi| \mathrm{K}_{k}^{\dagger},
$$

\footnotetext{
${ }^{3}$ Operation $\mathcal{J}$ is a map, $\mathcal{J}: \mathbf{T}\left(\mathbf{H}_{\tau}\right)_{1}^{+} \mapsto \mathbf{T}\left(\mathbf{H}_{\tau}\right)_{1}^{+}$, see Ref. [16].
} 
where

$$
\mathrm{K}_{k}=\sum_{l}\left|\varphi_{k l}\right\rangle\left\langle\phi_{k l}\right|
$$

One can show that this relation is general,

$$
\mathcal{I}(X)(\mathrm{T})=\sum_{o_{k} \in X} \mathrm{~K}_{k} \mathrm{TK}_{k}^{\dagger}
$$

for proof, see Ref. [16]. We have then

$$
\mathcal{I}(Y)(\mathcal{I}(X)(\mathrm{T}))=\sum_{o_{l} \in X} \mathrm{~K}_{l}\left(\sum_{o_{k} \in X} \mathrm{~K}_{k} \mathrm{TK}_{k}^{\dagger}\right) \mathrm{K}_{l}^{\dagger}=\sum_{o_{l} \in X} \sum_{o_{k} \in X}\left(\mathrm{~K}_{l} \mathrm{~K}_{k}\right) \mathrm{T}\left(\mathrm{K}_{l} \mathrm{~K}_{k}\right)^{\dagger} .
$$

Eq. (6.8) would be satisfied if

$$
\mathrm{K}_{l} \mathrm{~K}_{k}=\mathrm{K}_{k} \delta_{k l},
$$

which is in general not the case.

Let us therefore restrict ourselves to measurement couplings satisfying

$$
\phi_{k l}=\varphi_{k l}
$$

This model is called von Neumann premeasurement because it was first described in Ref. [54] 4 .

For von Neumann premeasurement, the operator $\mathrm{K}_{k}$ is the projection $\mathrm{E}_{k}^{\mathrm{O}}$ on the eigenspace of $o_{k}$,

$$
\mathrm{K}_{k}=\sum_{l}\left|\phi_{k l}\right\rangle\left\langle\phi_{k l}\right|
$$

and Eq. ([6.9) is satisfied. Thus, von Neumann premeasurement is a special case of repeatable premeasurement.

The vector states $\varphi_{k}^{1}$ given by Eq. (6.4) are orthonormal for von Neumann premeasurements. Thus, the final state of the apparatus given by Eq. (6.5) reduces to (6.6). This is, however, not sufficient for the objectification requirement to be satisfied. The right-hand side of Eq. (6.6) must be the gemenge structure of the state. If so, then Rule 11 implies that the final state of the composite system $\mathcal{S}+\mathcal{A}$ must be

$$
\sum_{j} p_{j} \mathrm{~T}_{j}^{\prime} \otimes\left|\psi_{j}\right\rangle\left\langle\psi_{j}\right|
$$

where $T_{j}^{\prime}$ are some states of $\mathcal{S}$. But $\Phi_{\text {end }}$ of $(6.3)$ is a vector state. State operators of vector states admit only trivial decomposition. Hence, the objectification requirement is not satisfied for von Neumann premeasurements, and it is therefore not a measurement.

\footnotetext{
${ }^{4}$ In fact, von Neumann premeasurement is slightly more general in the sense that it is a premeasurement of a function $f(\mathrm{O})$, where $f$ need not be bijective, cf. Ref. [16].
} 
An analogous difficulty holds for more general models of premeasurement described in Ref. [16] and the book contains more general no-go theorems. This is called problem of objectification. The conclusion of Ref. [16] is that the von Neumann model of premeasurement, together with its generalisations, does not work as a model of measurement. Von Neumann himself postulated that measurements define another, non-unitary and indeterministic kind of evolution in which $\mathcal{S}$ randomly jumps into one of the eigenstates of the measured observable (Ref. [54], pp. 217, 351). This was called collapse of the wave function by Bohm (Ref. [10], p. 120).

\subsection{Comparison with real experiment. Importance of detectors}

The theoretical models of the previous section ought to describe and explain at least some aspects of real experiments. This section will try to go into all experimental details that can be relevant to our theoretical understanding.

First, we briefly collect what we shall need about detectors. Microsystem $\mathcal{S}$ to be detected interacts with the sensitive matter of the detector so that some part of energy of $\mathcal{S}$ is transferred to the detector. Mostly, $\mathcal{S}$ interacts with some subsystems of the sensitive matter exciting each of them because the excitation energy is much smaller than the energy of $\mathcal{S}$. The resulting subsystem signals are collected, or amplified and collected so that they can be distinguished from noise. For example, in ionisation detectors, many atoms or molecules of the sensitive matter are turned into electron-ion pairs. If the energy of $\mathcal{S}$ is much higher than the energy of one ionisation, say about $10 \mathrm{eV}$, then many electron-ion pairs are produced and the positive as well as the negative total charge is collected at electrodes [45].

In the so-called cryogenic detectors [68], $\mathcal{S}$ interacts, e.g., with superheated superconducting granules by scattering off a nucleus in a granule and the phase transition from the superconducting into the normally conducting phase of only one granule can lead to a perceptible electronic signal. A detector can contain very many granules (typically $10^{9}$ ) in order to enhance the probability of such scattering if the interaction between $\mathcal{S}$ and the nuclei is very weak (weakly interacting massive particles, neutrino). Modern detectors are constructed so that their signal is electronic. For example, to a scintillating matter, a photomultiplier is attached, etc., see Ref. 45].

In any case, in order to make a detector respond $\mathcal{S}$ must lose some of its energy to the detector. The larger the loss, the better the signal. Thus, most detectors are built in such a way that $\mathcal{S}$ loses all its kinetic energy and is absorbed by the detector (in this way, also its total momentum can be measured). Let us call such detectors absorbing. If the bulk of the sensitive matter is not large enough $\mathcal{S}$ can 
leave the detector after the interaction with it, in which case we call the detector non-absorbing. Observe that a detector is absorbing even if most copies of $\mathcal{S}$ leave the detector without causing a response but cannot leave if there is a response (e.g., neutrino detectors).

Suppose that $\mathcal{S}$ is prepared in such a way that it must cross a detector. Then, the probability of the detector response is generally $\eta<1$. We call a detector ideal, if $\eta=1$.

An important assumption, corroborated by all experiments, is that a real detector either gives a signal or remains silent in each individual registration. This corresponds here to the objectification requirement.

After these preparatory remarks, consider a kind of repeatable premeasurement that is often described in textbooks (see, e.g., Ref. [56], p. 27, where it is called 'repeatable test'), for example a Stern-Gerlach-like measurement of spin. Let coordinate system $\left\{x^{1}, x^{2}, x^{3}\right\}$ be chosen. Silver atoms evaporate in an oven $\mathcal{O}$, form a beam $B_{0}$ along $x^{2}$-axis passing through a velocity selector $\mathcal{V}$, and then through an inhomogeneous magnetic field produced by device $\mathcal{M}_{1}$. $\mathcal{M}_{1}$ splits $B_{0}$ into two beams, $B_{1+}$ and $B_{1-}$, of which $B_{1+}$ is associated with positive and $B_{1-}$ with negative spin $x^{1}$-component, the corresponding vector states being denoted by $|1+\rangle$ and $|1-\rangle$. Beam $B_{1-}$ is blocked off by a screen. This is the preparatory part of the experiment.

Next, beam $B_{1+}$ runs through another magnetic device, $\mathcal{M}_{3}^{(1)}$ with centre at $\vec{x}_{(1)}$ and finally strike an array of ideal detectors $\left\{\mathcal{A}_{k}^{(1)}\right\}$ placed and oriented suitably with respect to $\mathcal{M}_{3}^{(1)}$. Two detectors of array $\left\{\mathcal{A}_{k}^{(1)}\right\}$ respond, let us denote them by $\mathcal{A}_{+}$and $\mathcal{A}_{-}$, revealing the split of $B_{1+}$ into two beams, $B_{3+}$ and $B_{3-}$, caused by $\mathcal{M}_{3}^{(1)}$. Let the orientation of $\mathcal{M}_{3}^{(1)}$ be such that $B_{3+}$ corresponds to positive and $B_{3-}$ to negative spin $x^{3}$-component, the associated states of silver atoms being $|3+\rangle$ or $|3-\rangle$. The beams $B_{3+}$ and $B_{3-}$ are spatially sufficiently separated when they arrive at the detectors so that they hit different detectors $\mathcal{A}_{+}$and $\mathcal{A}_{-}$. In this way, the values of the quantum mechanical centres of mass of silver atoms at the moment of their interaction with the detectors are measured. These centres of mass denoted by $\vec{x}_{3+}$ and $\vec{x}_{3-}$ are registered by the detectors in a rather coarse-grained way, which is, however, perfectly sufficient to determine the spins of the atoms. Pointer values are the electronic responses of detectors $\mathcal{A}_{+}$and $\mathcal{A}_{-}$. Let us call experiment I what is performed by $\mathcal{O}, \mathcal{V}, \mathcal{M}_{1}, \mathcal{M}_{3}^{(1)}$ and $\left\{\mathcal{A}_{k}^{(1)}\right\}$.

Let us now remove $\left\{\mathcal{A}_{k}^{(1)}\right\}$, place device $\mathcal{M}_{3}^{(2)}$ of the same macroscopic structure and orientation as $\mathcal{M}_{3}^{(1)}$ with centre position $\vec{x}_{(2)}$ in the way of $B_{3+}$ so that $B_{3-}$ passes by and arrange array $\left\{\mathcal{A}_{k}^{(2)}\right\}$ so that it has the same relative position with respect to $\mathcal{M}_{3}^{(2)}$ as $\left\{\mathcal{A}_{k}^{(1)}\right\}$ had with respect to $\mathcal{M}_{3}^{(1)}$. Now, only one detector will respond, namely that which is approximately at the position $\vec{x}_{3+}-\vec{x}_{(1)}+\vec{x}_{(2)}$. Let 
us call experiment II what is performed by $\mathcal{O}, \mathcal{V}, \mathcal{M}_{1}, \mathcal{M}_{3}^{(1)}, \mathcal{M}_{3}^{(2)}$ and $\left\{\mathcal{A}_{k}^{(2)}\right\}$. The result of experiment II is described in Ref. [56] as 'two consecutive identical tests following each other with a negligible time interval between them ... yield identical outcomes'.

Clearly, experiment II does not consist of two copies of experiment I performed after each other. The only repetition is that device $\mathcal{M}_{3}^{(2)}$ is placed after $\mathcal{M}_{3}^{(1)}$ and has the same structure and orientation with respect to its incoming beam $B_{3+}$ as $\mathcal{M}_{3}^{(1)}$ has with respect to $B_{1+}$. Device $\mathcal{M}_{3}^{(1)}$ splits $B_{1+}$ into $B_{3+}$ and $B_{3-}$ but $\mathcal{M}_{3}^{(2)}$ does not split $B_{3+}$. One may say that it leaves $B_{3+}$ unchanged. Let us define the action of device $\mathcal{M}_{3}^{(k)}$ together with the choice of $( \pm)$-beam for each $k=1,2$ as a test (in the sense of Ref. [56]) or a premeasurement. Let the outcomes be the thought responses of imaginary detectors placed in the way of the chosen beam. Then the (counterfactual) outcomes can be assumed to be identical indeed and we have an example of repeatable premeasurement that satisfies Definition 22.

The procedures defined in this way are premeasurements that can be described by von Neumann model. The value of the spin is transformed by the magnetic field into the value $\vec{x}_{3+}$ of its centre-of mass-positions that can be considered as the eigenvalues of the pointer observable associated with effects $|3 \pm\rangle\langle 3 \pm|$. However, the premeasurement cannot be considered as an instance of registration because it does not give us any information about the silver atoms. Try to suppose, e.g., that the arrangement measures effects $|3 \pm\rangle\langle 3 \pm|$ depending on which of the outgoing beams is chosen. Now, how can we recognise whether the outcome is 'yes' or 'not'? There is no change of a classical property of an apparatus due to its interaction with a microsystem that would indicate which of the values $\vec{x}_{3+}$ and $\vec{x}_{3}$ - results. But premeasurement is allowed not to give definite responses by each individual action. To obtain definite values, additional detectors are needed. Without the additional detector, however, this real premeasurement is not a measurement.

The above observations about the role of detectors seem to be obvious. It is a strange fact, however, that the detectors are neglected or deemed irrelevant in old as well as in modern theoretical accounts of quantum measurement. For example, Ref. [55], p. 64, describes a measurement of energy eigenvalues with the help of scattering similar to Stern-Gerlach experiment, and it explicitly states:

We can consider the centre of mass as a 'special' measuring apparatus...

Similarly, Ref. [56], p. 17 describes Stern-Gerlach experiment:

The microscopic object under investigation is the magnetic moment $\mu$ of an atom.... The macroscopic degree of freedom to which it is coupled in this model is the centre of mass position $\mathbf{r} \ldots$. I call this degree of freedom macroscopic because different final values of $\mathbf{r}$ can be directly 
distinguished by macroscopic means, such as the detector... From here on, the situation is simple and unambiguous, because we have entered the macroscopic world: The type of detectors and the detail of their functioning are deemed irrelevant.

In our opinion, this notion of measurement is erroneous and leads directly to von Neumann model and to the problem of quantum measurement.

Suppose next that there are non-absorbing ideal detectors that do not disturb the spin state of the atom. This might work, at least approximately. Let experiment I' be the same as I with the only change that the array $\left\{\mathcal{A}_{k}^{(1)}\right\}$ is replaced by $\left\{\mathcal{A}_{k}^{p(1)}\right\}$ containing the non-absorbing detectors. Let experiment II' starts as I' and proceeds as II but with $\left\{\mathcal{A}_{k}^{(2)}\right\}$ replaced by $\left\{\mathcal{A}_{k}^{p(2)}\right\}$ made from the non-absorbing detectors. Clearly, the action of $\left(\mathcal{M}_{3}^{(j)}+\left\{\mathcal{A}_{k}^{p(j)}\right\}\right)$ for each $j=1,2$ is a repeatable premeasurement according to Definition 22, and it is even a repeatable measurement because of the responses of the real detectors, but it definitely cannot be described by a von Neumann theoretical model. For the detectors to respond, some part of the energy of the atoms is needed, so that condition (6.10) is not satisfied.

An interesting difference emerges here between what we can say about the system (silver atom) on the one hand and about states on the other in their relation to the beams $B_{3+}$ and $B_{3-}$. Whereas $B_{3+}$ is associated with $|3+\rangle$ and $B_{3-}$ with $|3-\rangle$, each atom is in a linear superposition of the two states $|3+\rangle$ and $|3-\rangle$ that equals to the prepared state $|1+\rangle$. One cannot even say that all atoms in beam $B_{3+}$ are in state $|3+\rangle$ because no atom is just in $B_{3+}$. Unlike the states, the atoms are not divided between the beams. Indeed, the two beams could be guided so that no detectors are in their two ways and that they meet each other again. Then, they would interfere and if the two ways are of equal length, so that no relative phase shift results, the original state $|1+\rangle$ would result. This would happen even if the beams are very thin, containing always at most one silver atom. Hence, each atom in its wave aspect had to go both ways simultaneously.

Let us however observe that each of the beams $B_{3+}$ and $B_{3-}$ by itself behaves as if it were a prepared beam of silver atoms in a known state, which are $|3+\rangle$ and $|3-\rangle$, respectively. The fact that we place some arrangement $\mathcal{A}$ of devices that do not contain any detector in the way of beam $B_{3+}$ and leave $B_{3-}$ alone justifies our use of state in $|3+\rangle$ in all calculations of what will be the outcome after arrangement $\mathcal{A}$ is passed. The voluntary element of beam choice in this experiment can be interpreted as a preparation or as a reselection of ensemble, but not as a collapse of the wave function. Indeed, the whole outcome will be a linear superposition of states in each of the two beams at the time the upper beam passes $\mathcal{A}$. Only if we put any detector after $\mathcal{A}$ or, for that matter, a detector or just a screen into the way of $B_{3-}$, then something like a collapse of the wave function can happen. 
As yet, we have considered only the so-called direct registrations. A complete theory of registration must distinguish two kinds of registration.

A direct registration first manipulates $\mathcal{S}$ by classical fields and screens so that the prepared beam is split into spatially separated beams, each of which associated with one eigenvalue of the registered observable. Then, there is a set of detectors each of which can be hit by only one beam.

An indirect registration, such as scattering or QND measurement (see e.g. [13] and [56] p. 400), lets $\mathcal{S}$ interact with an auxiliary microsystem $\mathcal{S}_{1}^{\prime}$ and it is only $\mathcal{S}_{1}^{\prime}$ that is then subject to a direct registration. For the measurement to be QND, several further conditions must be satisfied, but this does not concern us here. After a QND procedure, $\mathcal{S}$ remains available to another one: another system $\mathcal{S}_{2}^{\prime}$ of the same type as $\mathcal{S}_{1}^{\prime}$ interacts with $\mathcal{S}$ and is then directly registered etc. Information given by the detectors of the direct registrations reveals also something about $\mathcal{S}$. Thus, detectors are necessary for indirect registrations, too.

The analysis of the present section motivates the following generalisation. First, an arrangement of devices that acts in agreement with von Neumann model of direct premeasurement is not a registration apparatus. Second:

Assumption 6 Any registration apparatus for microsystems must contain at least one detector and every reading of a pointer value is a signal from a detector.

Assumption 6 seems to be obvious but it has far reaching consequences.

As explained in Sec. 2.2.4, a preparation of a quantum object $\mathcal{S}$ of type $\tau$ must separate it from other systems of type $\tau$ in a space domain $D$. Assumption 6 implies, however, that this need not be sufficient for $\mathcal{S}$ to be an object on which registrations can be performed. Indeed, on the one hand, any direct registration first manipulates $\mathcal{S}$ individually by fields and screens and then detect it by a detector. For example, the scattering of electrons on a crystal of graphite can be described as manipulating each electron by an effective potential (=field). Also, $\mathcal{S}$ must have sufficient kinetic energy to excite the detector. On the other hand, an indirect registration on $\mathcal{S}$ is only possible if $\mathcal{S}$ is sufficiently isolated so that the auxiliary microsystem must interact with $\mathcal{S}$ rather than with any other microsystem.

It follows: For $\mathcal{S}$ to have any observable, it must be prepared

- in a domain $D$ that separates $\mathcal{S}$ from other microsystems not necessarily of the same type

- so that either $\mathcal{S}$ is available to manipulation by fields and screen 5 and has sufficient kinetic energy to excite a detector, in the case of a direct registration,

\footnotetext{
${ }^{5} \mathrm{~A}$ screen can be theoretically described as a boundary condition that absorbs everything and releases nothing.
} 
- or $\mathcal{S}$ is the only microsystem that can interact with the auxiliary microsystem in the case of an indirect one.

Definition 16 and Assumption 6 imply that both preparations and registrations of microsystem $\mathcal{S}$ are processes during which the separation status of $\mathcal{S}$ changes. In particular, in order that observable $\mathrm{E}$ of any microsystem $\mathcal{S}$ be registered, $\mathcal{S}$ must enter the sensitive matter part $\mathcal{M}$ of the corresponding detector. $\mathcal{M}$ contains subsystems of the same type as those that are also contained in $\mathcal{S}$, either because they were there already before the detection started or because $\mathcal{M}$ becomes polluted by them during the experiment. Moreover, $\mathcal{M}$ hinders individual manipulation by fields and screens that would be necessary for each given observable of $\mathcal{S}$ to be registered.

Assumption 6 also leads to restrictions on observables of macroscopic systems. In general, a macroscopic system $\mathcal{A}$ is a composite quantum system with very many different microsystem constituents. One can subdivide these microsystems into type classes. Consider first observables that concern properties of microscopic subsystem $\mathcal{S}$ of type $\tau$. If we apply the basic rules of observable construction for systems of identical microsystems (Sec. 2.2.1), then e.g. the position and momentum of any individual microsystem $\mathcal{S}$ are not observables of $\mathcal{A}$ but 'collective' operators such as

$$
\sum_{k} a\left(\vec{x}_{k} ; \vec{x}_{k}^{\prime}\right)
$$

where $a\left(\vec{x}_{k} ; \vec{x}_{k}^{\prime}\right)$ is the kernel of an operator in $Q$-representation acting on $k$-th subsystem of type $\tau$, could be. Suppose that there is an apparatus $\mathcal{B}$ suitable to measure $a\left(\vec{x}_{k} ; \vec{x}_{k}^{\prime}\right)$ on an individually prepared system $\mathcal{S}$. One can imagine that applying $\mathcal{B}$ to $\mathcal{A}$ in some way similar to that described in Sec. 2.2.2 would measure a $a_{\text {col }}$ because all subsystems of type $\mathcal{S}$ would automatically contribute to the result of the measurement. However, in Sec. 2.2.2, the registration apparatus was applied to individually prepared subsystems. It follows from Assumption 6 that the apparatus $\mathcal{B}$ cannot be applied to $\mathcal{A}$ in this way because none of the identical subsystems of type $\tau$ is prepared individually. Readings of $\mathcal{B}$ are signals of its detector and for any detector to register $\mathcal{S}, \mathcal{S}$ must be isolated to be manipulable, have sufficient kinetic energy, etc. Hence, to measure collective observable (6.11), we need a method that makes measurements directly on $\mathcal{A}$.

This holds for direct registrations. As an example of an indirect one consider scattering $X$-rays off a crystal $\mathcal{A}$. By it, relative positions of its nuclei can be recognised (an indirect registration). But rather than a position of an individual nucleus it is a space dependence of the average nuclear density due to all nuclei. Such an average nuclear density could be obtained with the help of an operator of the symmetrised form (6.11). In general, scattering of a microsystem $\mathcal{S}^{\prime}$ off 
a macrosystem $\mathcal{A}$ can be determined in terms of a potential $V_{k}\left(\vec{x}, \xi, \vec{x}_{k}, \xi_{k}\right)$ that describes the interaction between $\mathcal{S}^{\prime}$ and one of the microscopic subsystems of $\mathcal{A}$. The whole interaction Hamiltonian is then the sum analogous to (6.11),

$$
\sum_{k} V_{k}\left(\vec{x}, \xi, \vec{x}_{k}, \xi_{k}\right)
$$

which however extends over all microscopic subsystems of $\mathcal{A}$ that can interact with $\mathcal{S}^{\prime}$. It is also important to realise that there are very few interactions that can supply potentials useful for practical experiment.

Other examples have to do with additive quantities, such as momentum and angular momentum. Total values of these quantities can be measured and they are of the form (6.11), only the sum must extend to all microscopic subsystems of $\mathcal{A}$ independently of type.

We notice, first, that an observable $a\left(\vec{x}_{k} ; \vec{x}_{k}^{\prime}\right)$ of a microsystem $\mathcal{S}$ can be promoted to a collective observable (6.11) of $\mathcal{A}$ if $\mathcal{A}$ itself admits a direct or indirect registration of it, which happens only in special and seldom cases. Second, such a collective observable is still too 'sharp', because only some averages with rather large variances can be observed. It is impossible to obtain its single eigenvalues as results of registration (for an example, see Ref. [56], p.181).

A different case is the kinetic energy of $\mathcal{S}$. Again, it cannot be measured by the method kinetic energy is measured on individual systems of type $\mathcal{S}$. In the special case that $\mathcal{A}$ is in thermodynamic equilibrium, the average of the kinetic energy of $\mathcal{S}$ would have the meaning of $1 / k_{B}$ times the temperature of $\mathcal{A}$. Hence, a viable method to measure the average is to measure the temperature of $\mathcal{A}$. Again, this is a very special case that works only under specific conditions.

Thus, one of the consequences of Assumption 6 are principal and severe limits on promotion of POV measures to observables.

\subsection{A simple modification of BCL model}

Sec. 6.2 motivated the first idea relevant for the present section, namely that the reading of a registration apparatus for microsystems is in fact an electronic signal from a detector. This gives us much clearer notion of registration apparatus. The second relevant idea comes from Sec. 2.2.4, namely that fixed-status quantum mechanics (FSQM) has its limits. This consequence of general rules of standard quantum mechanics about indistinguishable microsystems leads to a significant modification of quantum theory of measurement. The necessary changes are:

1. Each preparation of microsystem $\mathcal{S}$ must give $\mathcal{S}$ a non-trivial separation status 
$D, D \neq \emptyset$. Thus, a quantum object comes into being only in preparation 6 .

2. Object $\mathcal{S}$ must be prepared so that it can then be manipulated and controlled by devices within $D$ such as electric and magnetic fields. This can be viewed as additional condition on separation status, see Ref. [32].

3. Macrosystem $\mathcal{A}$ such as a blocking screen, a scattering target or a detector that contains microsystems indistinguishable from $\mathcal{S}$ or from some subsystems of $\mathcal{S}$ must lie behind the boundary of $D$. Other microsystems of the same type as $\mathcal{S}$ that may be in $D$ due, e.g., to imperfect vacuum must not disturb registration of $\mathcal{S}$. Corrections to FSQM description of the behaviour of the composed system $\mathcal{S}+\mathcal{A}$ due to a necassary separation-status change of $\mathcal{S}$ must be carefully chosen.

The usual method of FSQM is to specify initial states of both $\mathcal{S}$ and $\mathcal{A}$ before their interaction, choose some appropriate interaction Hamiltonian and calculate the corresponding unitary evolution of the composed system $\mathcal{S}+\mathcal{A}$ ignoring the problem with separation-status change. As shown in Sec. 6.1, the results are wrong for registration apparatuses. The purpose of this section is to make some corrections. First, we must modify and extend BCL model.

Let system $\mathcal{S}$ of type $\tau$ be prepared as a quantum object of separation status $D$ in a domain $D$. Let $\mathrm{O}$ be the observable to be registered with eigenvalues $o_{k}$ and eigenvectors $\phi_{k l}$ as in Sec. 6.1. Let $\mathcal{S}$ be prepared in state $\phi$ and manipulated so that its component eigenstates of $\mathrm{O}$ become spatially separated.

Next, we construct a sufficiently detailed model of detector $\mathcal{A}$. Let $\mathcal{A}$ be an array of $N$ ideal monoatomic-gas ionisation detectors $\mathcal{A}_{k}$ similar to that of Sec. 6.2. Let index $k$ enumerate the detectors and let the sensitive matter of each detector have separation status $D_{k}$ where $D_{k} \cap D_{l}=\emptyset$ for all $k \neq l$ and $D_{k} \cap D=\emptyset$ for all $k$. Let the sensitive matter be a system of identical atoms. Let each atom be modelled by a particle with mass $\mu$, spin zero and a further degree of freedom, ionisation, with two values, 'non-ionised' and 'ionised'. We simplify the model further by assuming that the ionisation and translation degrees of freedom define two different formal subsystems, $\mathcal{A}_{\text {ion }}$ and $\mathcal{A}_{\text {tra }}$ which can be decomposed in subsystems $\mathcal{A}_{\text {ion }}^{k}$ and $\mathcal{A}_{\text {tra }}^{k}$ of $\mathcal{A}_{k}$. Let $\chi_{k n}$ be the state describing $n$ ionised atoms in $k$ th detector. The states

$$
\prod_{k} \otimes \chi_{k n(k)}
$$

for all $n(k)$ 's form a basis of the Hilbert space of $\mathcal{A}_{\text {ion }}$, where $n(k)$ is a map from $\{1, \cdots, N\}$ into non-negative integers. Let us assume that the initial state of $\mathcal{A}_{\text {ion }}$

\footnotetext{
${ }^{6}$ There is no problem for realism here. Even in classical physics, we often experiment with objects that are set up in the laboratory and need not exist before the experiment.
} 


$$
\psi=\prod_{k} \otimes \chi_{k 0}
$$

the perfectly non-ionised state. We can further assume that the initial state $\mathrm{T}_{\text {tra }}$ of $\mathcal{A}_{\text {tra }}$ is close to a state of maximum entropy with sufficiently low temperature so that ionisations due to atomic collisions have a very low probability.

Let each $\mathcal{A}_{k}$ further contains family $\mathcal{S}_{k}^{\prime}$ of systems of type $\tau$ identical with $\mathcal{S}$ and let the number of such subsystems in $\mathcal{S}_{k}^{\prime}$ be $M_{k}$. Thus, $\mathcal{A}_{k}=\mathcal{A}_{\text {ion }}^{k}+\mathcal{A}_{\text {tra }}^{k}+\mathcal{S}_{k}^{\prime}$ Let the subsystems of $\mathcal{S}_{k}^{\prime}$ be distributed over the sensitive matter of the $k$-th detector so that the separation status of $\mathcal{S}_{k}^{\prime}$ is $D_{k}$. We assume that the subsystems of $\mathcal{S}_{k}^{\prime}$ do not interact with each other, with the rest of the detector and with $\mathcal{S}$. Let the state of $\mathcal{S}_{k}^{\prime}$ be $\mathrm{T}_{k}$. Hence, $\mathrm{T}_{k}$ satisfies

$$
\mathrm{T}_{k}=\mathrm{P}_{\tau}^{\left(M_{k}\right)} \mathrm{T}_{k} \mathrm{P}_{\tau}^{\left(M_{k}\right)}
$$

where $\mathrm{P}_{\tau}^{\left(M_{k}\right)}$ is the projection to the symmetric or antisymmetric subspace of $\mathbf{H}_{\mathcal{R}(\tau)}^{M_{k}}$, depending on $\tau$, see Sec. 2.2.1.

Let the measurement coupling be a coupling between $\mathcal{S}$ and the ionisation degree of freedom of each atom in the sensitive matter of the whole array. That is, $\mathcal{S}$ interacts directly only with $\mathcal{A}_{\text {ion }}$. In a single detector, after the ionisation of the first atom, all subsequent ionisations lie along a ray track inside the same detector. An explanation of the fact that e.g. a spherical wave can produce a straight track is given in Ref. [53], where it is shown that the position of the track head, the first ionisation of the track, determines the track. This can be considered as a necessary property of every measurement coupling that is possible in the case considered here.

Let our first attempt at the new rule be based on the assumption that the dynamical rule of the standard quantum mechanics holds. Under this assumption, let the measurement coupling fulfils the conditions of the BCL model, so that, if systems $\mathcal{S}_{k}^{\prime}$ did not exist, we would be lead to Eq. (6.2), where $\psi$ is given by Eq. (6.12),

$$
\psi_{k}=\left(\prod_{j=1}^{k-1} \otimes \chi_{j 0}\right) \otimes\left(\sum_{n} a_{n} \chi_{k n}\right) \otimes\left(\prod_{j=k+1}^{N} \otimes \chi_{j 0}\right)
$$

and $a_{n}$ are coefficients independent of $k$ satisfying $\sum_{n}\left|a_{n}\right|^{2}=1$. This is again a simplifying assumption: each $\mathcal{S}$ creates always the same ionisation state in any detector. Then Eqs. (6.3) and (6.4) are valid. Now, we can define the observable A by its eigenstates (6.12) with eigenvalue 0 and $\psi_{k}$ 's with eigenvalues $o_{k}$ assuming $o_{k} \neq 0$ for all $k$.

In Sec. 6.1, states $\psi_{k}$ were called 'end states' of $\mathcal{A}$ and A was called 'pointer observable'. Here, we prefer to call A trigger observable and $\psi_{k}$ trigger states because 
there is a further evolution of $\mathcal{A}$ independent of $\mathcal{S}$ that leads from $\psi_{k}$ to the concentration of charges at the electrodes and an electronic signal of $k$-th detector. This is due to a coupling between $\mathcal{A}_{\text {ion }}$ and $\mathcal{A}_{\text {tra }}$ mediated by the electrostatic field of the electrodes: ionised atoms move in a different way than the non-ionised ones. This motion leads to atom collisions and further ionisation in a complicated irreversible process. Only then, the true end states with true pointer values are achieved. The pointer values are some averages with some variances, in agreement with the expectation of Refs. [29, 30]. However, what is important for us happened already at the trigger stage and we can ignore the evolution from a trigger state to a detector signal.

Let further the support of trigger states $\varphi_{k l}$ of $\mathcal{S}$ be $D_{k}$ for all $k, l$. It follows then that,

$$
\left\langle\varphi_{k l} \mid \varphi_{m n}\right\rangle=\delta_{k m} \delta_{l n}
$$

Thus, we assume a special case of the BCL dynamics. Then, that Eq. (6.6) would hold.

The existence of systems $\mathcal{S}_{k}^{\prime}$ leads however to change of the separation status of $\mathcal{S}$ after $\mathcal{S}$ enters the sensitive matter of a detector. We can take this into account similarly as in Sec. 2.2.4 and replace state $\Phi_{\text {end }}$ of system $\mathcal{S}+\mathrm{A}_{\text {ion }}$ by a suitably entangled state $T_{\text {trig }}$ of system $\mathcal{S}+\sum_{k} \mathcal{S}_{k}^{\prime}+\mathcal{A}_{\text {ion }}$. However, many choices are possible, for example:

$$
\mathrm{T}_{\text {trig1 }}=\sum_{k l} \sqrt{p_{k} p_{l}} \nu^{2} \mathrm{P}_{\tau}^{\left(1+\sum_{k} M_{k}\right)}\left(\left|\varphi_{k}^{1}\right\rangle\left\langle\varphi_{l}^{1}\right| \otimes \mathrm{T}_{1} \otimes \cdots \otimes \mathrm{T}_{N}\right) \mathrm{P}_{\tau}^{\left(1+\sum_{k} M_{k}\right)} \otimes\left|\psi_{k}\right\rangle\left\langle\psi_{l}\right|,
$$

where $\nu$ is a suitable normalisation factor (projections do not preserve norm). State $\mathrm{T}_{\text {trig1 }}$ entails a maximal entanglement of identical systems within $\mathcal{S}+\sum_{k} \mathcal{S}_{k}^{\prime}$. This is not in agreement with Rule 14 because for each $k$, the separation status of system $\mathcal{S}_{k}^{\prime}$ is $D_{k}$ as is the support of the state $\left|\varphi_{k}^{1}\right\rangle$.

An attempt that entails a minimal entanglement of identical systems that is still compatible with our ideas on separation status is:

$$
\begin{aligned}
\mathrm{T}_{\text {trig2 }}=\sum_{k l} \sqrt{p_{k} p_{l}} \nu_{k} \nu_{l} \mathrm{~W}_{k l} \otimes \mathrm{T}_{1} \otimes \cdots \otimes \mathrm{T}_{k-1} \otimes \mathrm{T}_{k+1} \otimes \\
\cdots \otimes \mathrm{T}_{l-1} \otimes \mathrm{T}_{l+1} \otimes \cdots \otimes \mathrm{T}_{N} \otimes\left|\psi_{k}\right\rangle\left\langle\psi_{l}\right| .
\end{aligned}
$$

The form of the summands is valid just for non-diagonal terms $k<l$, but the sum must be understood as consisting of all non-diagonal terms, $k \neq l$, with operators

$$
\mathrm{W}_{k l}=\mathrm{P}_{\tau}^{\left(M_{k}+1\right)}\left(\mathrm{T}_{k} \otimes\left|\varphi_{k}^{1}\right\rangle\right)\left(\left\langle\varphi_{l}^{1}\right| \otimes \mathrm{T}_{l}\right) \mathrm{P}_{\tau}^{\left(M_{l}+1\right)}
$$

on the extended Hilbert space $\mathbf{H}_{\tau}^{1+M_{k}+M_{l}}$ of system $\mathcal{S}+\mathcal{S}_{k}^{\prime}+\mathcal{S}_{l}^{\prime}$, as well as the diagonal ones with the operator

$$
\mathrm{W}_{k k}=\mathrm{P}_{\tau}^{\left(M_{k}+1\right)}\left(\left|\varphi_{k}^{1}\right\rangle\left\langle\varphi_{k}^{1}\right| \otimes \mathrm{T}_{k}\right) \mathrm{P}_{\tau}^{\left(M_{k}+1\right)}
$$


on the Hilbert space of system $\mathcal{S}+\mathcal{S}_{k}^{\prime}$. Normalisation factor $\nu_{k}$ is determined by the condition

$$
\nu_{k}=\frac{1}{\sqrt{\operatorname{tr}\left[\mathrm{W}_{k k}\right]}} .
$$

Then, $T_{\text {trig2 }}$ satisfies the normalisation condition of a state. Indeed, the trace of a tensor product of operators is a product of the traces of the operator factors, see Proposition 24. As $\operatorname{tr}\left[\left|\psi_{k}\right\rangle\left\langle\psi_{l}\right|\right]=\delta_{k l}$, only the diagonal elements of the sum contribute. We have

Proposition 22 The trace of operator $\mathrm{W}_{k l}$ for $k \neq l$ is zero.

For proof, see next section.

Proposition 22 implies immediately that the trigger state of $\mathcal{A}_{\text {ion }}$, obtained from Eq. (6.14) and (6.13), is given by Eq. (6.6). However, the right-hand side of Eq. (6.6) is not the gemenge structure of the state because state $T_{\text {trig2 }}$ does not satisfy the conditions of Rule 11. Hence, our first choice of dynamics is not supported by experimental evidence.

Another attempt is to take just the diagonal elements of $\mathrm{T}_{\text {trig2 }}$ :

$$
\mathrm{T}_{\text {trig3 }}=\sum_{k} p_{k} \nu_{k}^{2} \mathrm{~T}_{1} \otimes \cdots \otimes \mathrm{T}_{k-1} \otimes \mathrm{W}_{k k} \otimes \mathrm{T}_{k+1} \otimes \cdots \otimes \mathrm{T}_{N} \otimes\left|\psi_{k}\right\rangle\left\langle\psi_{k}\right|
$$

State $\mathrm{T}_{\text {trig2 }}$ of $\mathcal{S}+\mathcal{A}_{\text {ion }}$ contains many correlations between systems $\mathcal{A}_{\text {ion }}$ and $\mathcal{S}+\sum_{k} \mathcal{S}_{k}^{\prime}$. The only correlations that remain in $\mathrm{T}_{\text {trig3 }}$ are, for each $k$, those between the trigger states $\psi_{k}$ of $\mathcal{A}_{\text {ion }}$ and states $\nu_{k}^{2} \mathrm{~W}_{k k}$ of system $\mathcal{S}+\mathcal{S}_{k}^{\prime}$.

States $\mathrm{T}_{\text {trig1 } 1}, \mathrm{~T}_{\text {trig2 }}$ or $\mathrm{T}_{\text {trig3 }}$ describe $\mathcal{S}$ being inside $\mathcal{A}$ and the registration is running. A part of this registration is the registration of $A$. Hence the observables that can be measured at this stage of the experiment must commute with $1 \otimes A$ to be jointly measurable with it. However, we have:

Proposition 23 Let B be a sharp observable of system of system $\mathcal{S}+\sum_{k} \mathcal{S}_{k}+\mathcal{A}_{\text {ion }}$ that commutes with $1_{\mathcal{S}+\sum_{k} \mathcal{S}_{k}} \otimes \mathrm{A}$. Then

$$
\operatorname{tr}\left[\mathrm{BT}_{\text {trig } 2}\right]=\operatorname{tr}\left[\mathrm{BT}_{\text {trig3 }}\right] .
$$

The proof of Proposition 23 will be given in the next section. Proposition 23 means that the states $T_{\text {trig2 }}$ and $T_{\text {trig3 }}$ cannot be distinguished from each other at this stage of the experiment. If the detector is non-absorbing, and system $\mathcal{S}$ is released from it, then the existing experimental evidence supports the notion that the state after the releasing is $\mathrm{T}_{\text {trig3 }}$ with the gemenge structure given by the right-hand side of Eq. (6.15) rather than $\mathrm{T}_{\text {trig2 }}$.

This motivates the following assumption: 
Rule 17 Let a microsystem $\mathcal{S}$ be detected by a detector $\mathcal{A}_{k}$ and let the measurement coupling modified as above satisfy Eq. (6.13). Then the state of $\mathcal{S}+\sum_{k} \mathcal{S}_{k}^{\prime}+\mathcal{A}_{\text {ion }}$ is

$$
\begin{aligned}
& \mathrm{T}_{\text {trig }}=\left(\sum_{k}\right) p_{k}\left(\left|\psi_{10}\right\rangle\left\langle\psi_{10}\right| \otimes \mathrm{T}_{1}\right) \otimes \cdots \otimes\left(\left|\psi_{k-10}\right\rangle\left\langle\psi_{k-10}\right| \otimes \mathrm{T}_{k-1}\right) \\
& \otimes\left|\sum_{n} a_{n} \chi_{k n}\right\rangle\left\langle\sum_{n} a_{n} \chi_{k n}\right| \otimes \nu_{k}^{2} \mathrm{P}_{\tau}^{\left(M_{k}+1\right)}\left(\left|\varphi_{k}^{1}\right\rangle\left\langle\varphi_{k}^{1}\right| \otimes \mathrm{T}_{k}\right) \mathrm{P}_{\tau}^{\left(M_{k}+1\right)} \\
& \otimes\left(\left|\psi_{k+10}\right\rangle\left\langle\psi_{k+10}\right| \otimes \mathrm{T}_{k+1}\right) \otimes \cdots \otimes\left(\left|\psi_{N 0}\right\rangle\left\langle\psi_{N 0}\right| \otimes \mathrm{T}_{N}\right) .
\end{aligned}
$$

State $T_{\text {trig }}$ satisfies two conditions:

1. The trigger state of $\mathcal{A}_{\text {ion }}$ has gemenge structure (6.6). That $\mathrm{T}_{\text {trig }}$ satisfies this condition follows from Rule 11.

2. It contains the same measurable correlation between systems $\mathcal{A}_{\text {ion }}$ and $\mathcal{S}+\mathcal{S}_{k}^{\prime}$ as $T_{\text {trig2 }}$. That $T_{\text {trig }}$ satisfies this condition follows from Proposition 23.

All other correlations are erased during the change of separation status of $\mathcal{S}+$ $\mathcal{A}$. What survives and what is erased is uniquely determined in this case by the modified BCL model and Rule 17. In particular, the probability reproducibility condition determines states $\varphi_{k l}$ from the initial state $\psi$ of $\mathcal{A}_{\text {ion }}$ uniquely and the initial state $\phi$ of $\mathcal{S}$ determines states $\varphi_{k}^{1}$ uniquely. Thus, the evolution from state $\left.|\phi\rangle\langle\phi| \otimes \mathrm{T}_{1} \otimes \cdots \otimes \mathrm{T}_{N}\right) \otimes\left|\psi_{0}\right\rangle\left\langle\psi_{0}\right|$ to state $\mathrm{T}_{\text {trig }}$ is non-unitary but still deterministic. Rule 17 is a new general rule which has to be added to quantum mechanics. To choose such a rule, we have looked at observations and experiments.

The correct interpretation of Rule 17 distinguishes two cases. If the detectors are absorbing, then state $T_{\text {trig }}$ evolves with $\mathcal{S}$ staying inside $\mathcal{A}$ and being lost always in one of the detectors. If they are non-absorbing, then state $T_{\text {trig }}$ evolves to

$$
\mathrm{T}_{\text {truend }}=\left(\sum_{k}\right) p_{k}\left|\varphi_{k}^{1}\right\rangle\left\langle\varphi_{k}^{1}\right| \otimes \mathrm{T}_{k}^{\prime},
$$

where $\mathrm{T}_{k}^{\prime}$ are the end states of $\mathcal{A}_{k}$ including the detector response. In this case, states $\nu_{k}^{2} \mathrm{~W}_{k k}$ evolved to the release of $\mathcal{S}$ in state $\varphi_{k}^{1}$ that is correlated with detector signals. Each release is understood as an instance of preparation and the whole procedure is a random mixture of these single preparations. In both cases, the end state of $\mathcal{A}_{\text {ion }}$ has then gemenge structure (6.6).

As an example of a system of non-absorbing detectors, the MWPC telescope for particle tracking can be mentioned [45]. It is a stack of the so-called multiwire proportional chambers (MWPC), which is arranged so that a particle runs through, 
exciting each of them. The resulting system of electronic signals contains the information about the particle track.

A registration by a non-absorbing detector is similar to a scattering of a microsystem by a macroscopic target. First, let us consider no-entanglement processes such as the scattering of electrons on a crystal of graphite with a resulting interference pattern [19] or the splitting of a laser beam by a down-conversion process in a crystal of $\mathrm{KNbO}_{3}$ (see, e.g., Ref. [51]). No-entanglement processes can be described by the following model. Let the initial state of the target $\mathcal{A}$ be $\mathrm{T}$ and that of the microsystem be $\phi$. We assume that the end state of the target is $\mathrm{T}^{\prime}$ and the end-state of the microsystem is $\varphi$ and that we have a unitary evolution:

$$
|\phi\rangle\langle\phi|\otimes \mathrm{T} \mapsto| \varphi\rangle\langle\varphi| \otimes \mathrm{T}^{\prime}
$$

The two systems are not entangled by their interaction, hence there is no necessity to divide the resulting correlations between $\mathcal{S}$ and $\mathcal{A}$ in what survives and what is erased. The end state is already of the form (6.17) and it has a trivial gemenge structure. In this way, our corrections to FSQM become trivial.

A more complicated case is an entanglement scattering. Let microsystem $\mathcal{S}$ in initial state $\phi$ be scattered by a macrosystem $\mathcal{A}$ in initial state $\mathrm{T}$ and let this lead to excitation of different microscopic subsystems of $\mathcal{A}$. Scattering of neutrons on spin waves in ferromagnets, transmutation of nuclei inside $\mathcal{A}$ or, for that matter, ionising an atom in a gas detector are examples. We have, therefore, a more general situation than that in which Rule 17 gives a unique result. It seems that the change of status must lead to survival of some correlations between $\mathcal{S}$ and $\mathcal{A}$ while some are erased. However, in this situation it has still to be investigated which is which. Clearly, the definitive general rule will depend on the two interacting systems and on the interaction Hamiltonian. More theoretical and experimental work is necessary to guess the general rule.

One can wonder whether a more detailed quantum mechanical model can be constructed of what happens during a change of separation status. The reason why this cannot be done within FSQM is that FSQM is not applicable to changes of separation status. Hence, just a new law added to FSQM is needed.

\subsubsection{Proofs of Propositions 22 and 23}

For the proofs, we shall need some simple properties of trace.

Proposition 24 1. Let operator $\mathrm{B}$ has a form $\mathrm{B}=\sum_{m n} B_{m n}|m\rangle\langle n|$, where $\{|n\rangle\}$ is some family of states. Then,

$$
\operatorname{tr}[\mathrm{B}]=\sum_{m n} B_{m n}\langle m \mid n\rangle
$$


2. Let operator $\mathbf{B}$ on Hilbert space $\mathbf{H}$ leave a subspace $\mathbf{H}_{1} \in \mathbf{H}$ invariant and acts trivially on $\mathbf{H}_{1}^{\perp}$. Then

$$
\operatorname{tr}_{\mathbf{H}}[\mathrm{B}]=\operatorname{tr}_{\mathbf{H}_{1}}[\mathrm{~B}] .
$$

3. Let three trace-class operator $\mathrm{B}, \mathrm{B}_{1}$ and $\mathrm{B}_{2}$ satisfy $\mathrm{B}=\mathrm{B}_{1} \otimes \mathrm{B}_{2}$. Then

$$
\operatorname{tr}[\mathrm{B}]=\operatorname{tr}\left[\mathrm{B}_{1}\right] \operatorname{tr}\left[\mathrm{B}_{2}\right] .
$$

Proof of Proposition 22 Our method is to expand operator $\mathrm{W}_{k l}$ in a basis and to use Proposition 24. The basis can be chosen as follows. In our model, states $\varphi_{k}^{1}$ and $\mathrm{T}_{k}$ have supports inside $D_{k}$. Hence, they can be expanded into a basis $\left\{\phi_{m}^{k}\right\}, m=$ $1,2, \cdots$ of $\mathbf{H}_{D_{k}}$. We can choose $\phi_{1}^{k}=\varphi_{k}^{1}$. Then, the family of vectors $\left\{\phi_{m}^{k}\right\} \cup\left\{\phi_{n}^{l}\right\}$ is a basis for the extended Hilbert space $\left(\mathbf{H}_{\tau}\right)^{1+M_{k}+M_{l}}$ of system $\mathcal{S}+\mathcal{S}_{k}^{\prime}+\mathcal{S}_{l}^{\prime}$. Any term in expansion of operator $\mathbf{W}_{k l}$ into basis $\left\{\phi_{m}^{k}\right\} \cup\left\{\phi_{n}^{l}\right\}$ will contain composite vectors

$$
\operatorname{Perm}\left[1+M_{k}\right]\left(\left|\phi_{1}^{k}\right\rangle \otimes\left|\phi_{m(1)}^{k}\right\rangle\right) \otimes \cdots \otimes\left|\phi_{m\left(M_{k}\right)}^{k}\right\rangle \otimes \operatorname{Perm}\left[M_{l}\right]\left(\left|\phi_{n(1)}^{l}\right\rangle \otimes \cdots \otimes\left|\phi_{n\left(M_{l}\right)}^{l}\right\rangle\right)
$$

left and

$$
\operatorname{Perm}\left[M_{k}\right]\left(\left\langle\phi_{r(1)}^{k}\left|\otimes \cdots \otimes\left\langle\phi_{r\left(M_{k}\right)}^{k}\right)\right| \otimes \operatorname{Perm}\left[1+M_{l}\right]\left(\left\langle\phi_{1}^{l}\right| \otimes\left\langle\phi_{s(1)}^{l}\right| \otimes \cdots \otimes\left\langle\phi_{s\left(M_{l}\right)}^{l}\right|\right)\right.\right.
$$

right, where $\operatorname{Perm}[N]$ is an arbitrary but fixed order permutation of $N$ states and the state choices $m(\cdot), n(\cdot), r(\cdot)$ and $s(\cdot)$ are mappings from the first $M_{k}$ or $M_{l}$ positive integers to positive integers (they need not be injections). Clearly, the two composite vectors are orthogonal for any permutations and state choices because there is one more element of $\left\{\phi_{m}^{k}\right\}$ in the product defining the first one than in the second one, and one more element of $\left\{\phi_{m}^{l}\right\}$ in the product defining the second one than in the first one. Hence, the trace is zero, Q.E.D.

Proof of Proposition 23 According to Sec. 6.1, $\left\{\psi_{k}\right\}$ together with $\psi$ is an orthonormal basis in a Hilbert space on which $\mathrm{A}$ is an observable,

$$
\mathrm{A}=\sum_{k} o_{k}\left|\psi_{k}\right\rangle\left\langle\psi_{k}\right|
$$

and all $o_{k}$ are different from each other.

Let $\left\{\Phi_{k}\right\}$ be a basis for the Hilbert space of system $\mathcal{S}+\sum_{k} \mathcal{S}_{k}^{\prime}$. Then, any observable B can be written as

$$
\mathrm{B}=\sum_{k l m n} B_{k l m n}\left|\Phi_{k}\right\rangle\left\langle\Phi_{m}|\otimes| \psi_{l}\right\rangle\left\langle\psi_{n}\right|
$$


In order to commute with $1 \otimes \mathrm{A}, \mathrm{B}$ has to be diagonal in $l$ and $n$ :

$$
\mathrm{B}=\sum_{k l m} B_{k l m}\left|\Phi_{k}\right\rangle\left\langle\Phi_{m}|\otimes| \psi_{l}\right\rangle\left\langle\psi_{l}\right|
$$

Then, we easily obtain

$$
\operatorname{tr}\left[\mathrm{T}_{\text {trig2 } 2} \mathrm{~B}\right]=\sum_{k m l} B_{k m l} p_{m} \nu_{m}^{2}\left\langle\Phi_{l}\left|\mathrm{~T}_{1} \otimes \cdots \otimes \mathrm{T}_{m-1} \otimes \mathrm{W}_{m m} \otimes \mathrm{T}_{m+1} \otimes \cdots \otimes \mathrm{T}_{N}\right| \Phi_{l}\right\rangle
$$

and the same result for $\operatorname{tr}\left[\mathrm{T}_{\text {trig3 }} \mathrm{O}^{\prime}\right]$, which proves Proposition 23. 


\section{Chapter 7}

\section{Conclusion}

Our careful study of quantum-mechanical practice, both experimental and theoretical, has lead us to quite a new picture of quantum mechanics. It differs from most current approaches in several points.

First, we do not consider a value of an observable of system $\mathcal{S}$ as a property of $\mathcal{S}$ but only as an indirect piece of information on such properties. Each value of observable is determined by both objective properties of $\mathcal{S}$ and the registration apparatus. Thus, we reject attempts to view all observables of $\mathcal{S}$, or at least some subsets of them, as objective properties of $\mathcal{S}$.

Second, we find a qualitative difference between quantum microsystems and large composite systems. In nature, microsystems occur in families of a fixed type, the elements of which are identical, and do not possess any individuality in strict sense. Sufficiently large composite systems are different for the following reason. The larger a composite system is, the less probable the existence of an identical system somewhere in the universe is.

Third, classical properties are objective quantum properties of macrosystems associated with states that are close to maximum entropy. We have shown how this principle is applied to classical mechanics by introducing a new class of states, the maximum-entropy packets.

Fourth, we give preparation a different and much greater significance than is usually assumed. In a preparation procedure, the prepared system gains its objective quantum properties such as states, gemenge structures, averages and variances of observables etc. so that it is justified to speak of a physical object. This is what we call quantum object. Next, a preparation must separate a microsystem from the set of identical microsystems, at least approximately so that it obtains a nontrivial separation status. Only then, it can be viewed as an individual system and a particular set of observables depending on the status becomes measurable on it. In this way, the standard rule for composition of identical microsystems 
can be weakened. This is justified by the idea of cluster separability. Finally, a preparation also separates the microsystem from all othe microsystems so that it can be individually manipulated by external fields or matter shields and registered by detectors.

Fifth, macrosystems, as well as generally large composite systems, have much less observables than one would expect according to standard quantum mechanics. There are two reasons why only very few observables concerning single constituents of such a system can be measured. On the one hand, the constituents may be elements of a large family of identical systems from which they are not separated by preparation and do not, therefore, possess any measurable observables of their own. On the other, they are not separated from other microsystems to be individually manipulable by fields and shields and registrable by detectors. The differences between macroscopic and microscopic systems that are the contents of the second, third and fifth points are not due to inapplicability of quantum mechanics to macroscopic systems. Just the opposite is true: they result from strict and careful application of standard quantum mechanics to macroscopic systems.

Sixth, we give registration a more specific form than is usually assumed. Any apparatus that is to register a microsystem directly contains a detector and the 'pointer' value that is read off the apparatus is a signal from the detector. We assume that each detector contains a macroscopic piece of sensitive matter with which the registered microsystem becomes unified and looses its object status. The post-Everett trend in the theory of quantum measurement is to avoid collapse of the wave function. In a sense, the present paper is heading in the opposite direction. We even replace the collapse by a more radical transformation, a change in microsystem kinematic description. This change is, in plain words, a kind of disappearance of a registered object during its registration. Nevertheless, our result for non-absorbing detectors and the collapse idea by von Neumann have some features in common.

Finally, standard quantum mechanics does not provide information about processes, in which the separation status of microsystems changes. Preparations and registrations belong to such processes. Unjustified application of standard rules to such processes leads to contradictions with experimental evidence. However, one can add new rules to quantum mechanics governing such processes without violating its logic.

The main purpose of Chap. 5 is to show how the new ideas on measurement problem work by studying well-understood, restricted class of physical conditions in which the following assumptions are a good approximation: (a) non-relativistic quantum mechanics, (b) measurement performed directly on microsystems, (c) a simple modification of BCL model of measurement and (d) pointer readings being signals from detectors. 
In our future work, we have gradually to remove the restrictions. First, we must turn to other models of measurement, for example to different (non-ideal) kinds of detectors or to the more realistic unitary premeasurements for which the nogo theorems such as Theorem 6.2.1 in Ref. [16], p. 76, hold. The main point is again that the state resulting from the unitary evolution contains information about properties of the composite system $\mathcal{S}+\mathcal{A}$ that could be measured only if more observables than $\mathrm{O}$ of $\mathcal{S}$ existed. Thus, a change of this illusory state analogous to that given by Rule 17 could be justified, it could again remove the objectification problem and the no-go theorems could be avoided. The exact division line between correlations that survive and those that are erased might again be determined by a careful analysis of observational facts.

Next, relativistic corrections have been neglected so that all notions and rules of non-relativistic quantum mechanics could be used. An extension of the present results to relativistic fields seems to be a realistic project.

Let us finish by giving a summary of how our theory changes quantum mechanics. Standard quantum mechanics has the so-called minimal interpretation (see, e.g., Ref. [16], p. 9). As measurement is concerned, it just postulates the probability reproducibility and objectification avoiding any deeper theory. It does not worry about origin of classical properties and about philosophy. Moreover, common practice of quantum mechanics does not take the symmetrisation or anti-symmetrisation over all identical systems seriously ignoring all unknown identical systems. This Minimal Quantum Mechanics has been in general use and it has been formidably successful. Our theory takes over Minimal Quantum Mechanics just giving a new foundation to the identical systems that justifies the common practice. To this, it adds a quantum theory of measurement that is motivated by the new ideas on identical systems and satisfies probability reproducibility and objectification requirements. Finally, it adds a realist interpretation and a theory of classical systems that do not contradict Minimal Quantum Mechanics.

\section{Acknowledgements}

The authors are indebted to Štefan Jánoš for help with experimental physics and to Heinrich Leutwyler for useful discussions. J. T. acknowledges partial support of the Ministry of Education of Czech Republic, research projects MSM6840770039 and LC06002. 


\section{Bibliography}

[1] E. Arthurs and J. L. Kelly, Jr., Bell System Tech. J. 44 (1965) 725.

[2] H. Araki and E. H. Lieb, Commun. Math. Phys. 18 (1970) 160.

[3] L. E. Ballentine, Quantum Mechanics. A Modern Development, World Scientific, Singapore, 2008.

[4] J. S. Bell, On Einstein-Podolsky-Rosen paradox, Physics bf 1 (19964) 195.

[5] J. S. Bell, Against 'measurement' in Sixty Two Years of Uncertainty, A. I. Miller (Ed.), Plenum, New York, 1990.

[6] J. S. Bell, Helv. Phys. Acta, 48 (1975) 93.

[7] J. S. Bell, On the problem of hidden variables in quantum mechanics, Rev. Mod. Phys. 38 (1966) 447.

[8] E. G. Beltrametti, G. Cassinelli and P. J. Lahti, J. Math. Phys. 31 (1990) 91.

[9] G. Birkhoff and J. von Neumann, Ann. of Math. 37 (1936) 823.

[10] D. Bohm, Quantum Theory, Prentice-Hall, Englewood Cliffs, 1951.

[11] P. Bóna, Acta Phys. Slov., 23 (1973) 149, 25 (1975) 3, 27 (1977) 101.

[12] M. Born, Phys. Blätter 11 (1955) 49.

[13] V. B. Braginsky and F. Ya. Khalili Rev. Mod. Phys. 681 (1996).

[14] Č. Brukner and M. Zukowski, Bell's Inequalities: Foundation and Quantum Communication, arXiv:0909.2611.

[15] J. Bub, Interpreting the Quantum World, Cambridge University Press, Cambridge, UK, 1999.

[16] P. Busch, P. J. Lahti and P. Mittelstaedt, The Quantum Theory of Measurement, Springer, Heidelberg, 1996. 
[17] C. Chevalley, in Niels Bohr and Contemporary Philosophy, ed. by J. Faye and H. Holse, Kluwer, Dordrecht, 1994.

[18] F. Coester, Int. J. Modern Phys. 17 (2003) 5328.

[19] C. Davisson and L. Germer, Phys. Rev. 30 (1927) 705.

[20] H. D. Doebner, P. Šťovíček and J. Tolar, Rev. Math. Phys. 13 (2001) 799. 705.

[21] F. Exner, Vorlesungen über die physikalischen Grundlagen der Naturwissenschaften, Deuticke, Leipzig, 1922.

[22] B. d'Espagnat, Veiled Reality, Addison-Wesley, Reading, 1995.

[23] J. Gemmer, M. Michel and G. Mahler, Quantum Thermodynamics. Emergence of Thermodynamic Behavior Within Composite Quantum Systems, LNP 657, Springer, Berlin, 2004.

[24] A. Bassi and G. Ghirardi, Phys. Letters A 275373 (2000).

[25] A. M. Gleason, J. Math. Mech. 6 (1957) 885.

[26] S. Goldstein, J. L. Lebowitz, R. Tumulka and N. Zanghi, Phys. Rev. Letters 96 (2006) 050403.

[27] D. M. Greenberger, M. A. Horne, A. Shimony, and A. Zeilinger, Bell's theorem without inequalities, Am. J. Phys. 58 (1990) 1131.

[28] R. Haag, Local Quantum Physics. Fields, Particles, Algebras, Springer, Berlin 1992.

[29] P. Hájíček and J. Tolar, Found. Phys. 39 (2009) 411.

[30] P. Hájíček, Found. Phys. 39 (2009) 1072.

[31] P. Hájíček, Quantum measurement problem and cluster separability, arXiv:1001.1827. To be published in Found. Phys., DOI: 10.1007/s10701-0109506-3.

[32] P. Hájíček, A new approach to quantum measurement problem: cluster separability, arXiv:1011.1732.

[33] L. Hardy, Quantum mechanics, local realistic theories, and Lorentz invariant realistic theories, Phys. Rev. Letters 68 (1992) 2981.

[34] K. Hepp, Helvetica Phys. Acta, 45 (1972) 237. 
[35] C. J. Isham, Lectures on Quantum Theory. Mathematical and Structural Foundations, Imperial College Press, London 1995.

[36] E. T. Jaynes, Probability Theory. The Logic of Science, Cambridge University Press, Cambridge (UK) 2003.

[37] B. Kayser and L. Stodolsky, Phys. Lett. B 395 (1995) 343.

[38] B. D. Keister and W. N. Polyzou, in Advances in Nuclear Physics, ed. J. W. Negele and E. Vogt, Plenum, New York 2002. Vol 20.

[39] C. Kittel, Introduction to Solid State Physics, Wiley, New York 1976.

[40] S. Kochen and E. P. Specker, J. Math. Mech. 17 (1967) 59.

[41] J. Kofler and Č. Brukner, Phys. Rev. Lett. 99 (2007) 180403.

[42] K. Kraus, States, Effects and Operations, Springer, Berlin, 1983; LNP 190.

[43] W: J. Mullin and F. Laloe, Classical phases and quantum angles in the description of interfering Bose-Einstein condensates, arXiv:0908.3012.

[44] A. J. Leggett, J. Phys.: Condens. Matter 14 (2002) R415.

[45] W. R. Leo, Techniques for Nuclear and Particle Physics Experiments, Springer, Berlin, 1987.

[46] J. M. Lévy-Leblond, in Group Theory and Its Applications, Vol. 2, ed. E. M. Loebl, Academic Press, New York 1941.

[47] N. Linden, S. Popescu, A. J. Short and A. Winter, arXiv:0812.2385.

[48] G. Ludwig, Foundations of Quantum Mechanics I, Springer, New York, 1983; Foundations of Quantum Mechanics II, Springer, New York, 1985.

[49] G. Ludwig, An Axiomatic Basis for Quantum Mechanics 1, Springer, Berlin, 1985; An Axiomatic Basis for Quantum Mechanics 2, Springer, Berlin, 1987.

[50] C. W. Misner, K. S. Thorn and J. A. Wheeler, Gravitation, Freeman, San Francisco, 1973.

[51] L. Mandel and E. Wolf, Optical Coherence and Quantum Optics, Cambridge University Press, Cambridge, 1995.

[52] Methods of Experimental Physics, Vol. 4, Part A, ed. by V. W. Hughes and H. L. Schultz, Academic Press, New York, 1967. 
[53] N. F. Mott, Proc. Roy. Soc. London Series A 126 (1929) 79.

[54] J. von Neumann, Mathematical Foundation of Quantum Mechanics, Princeton University Press, Princeton NJ, 1955.

[55] W. Pauli, Die allgemeinen Prinzipien der Wellenmechanik, Springer, Berlin, 1990.

[56] A. Peres, Quantum Theory: Concepts and Methods, Kluwer, Dordrecht, 1995.

[57] J. R. Pierce, An Introduction to Information Theory. Symbols, Signals and Noise, Dover, New York, 1980.

[58] C. Piron, Foundations of Quantum Physics, Benjamin, Reading 1976; C. Piron, Found. Phys. 2 (1972) 287.

[59] K. R. Popper, The Logic of Scientific Discovery, Hutchinson, London 1959.

[60] D. Poulin, Phys. Rev. A 71 (2005) 022102.

[61] S. Popescu, A. J. Short and A. Winter, arXiv:quant-ph/0511225 (2006).

[62] H. Primas, Chemistry, Quantum Mechanics and Reductionism. Springer, Berlin, 1983.

[63] M. Reed and B. Simon, Methods of Modern Mathematical Physics, Vol. I, Academic Press, New York, 1972.

[64] D. E. Rutherford, Proc. Roy. Soc. (Edinburgh), Ser. A, 62 (1947), 229; 63 (1951), 232.

[65] G. L. Sewell, Quantum Mechanics and its Emergent Macrophysics, Princeton University Press, Princeton, 2002.

[66] C. E. Shannon, Bell Syst. Tech. J. 27 (1948) 379, 623.

[67] A. Tonomura, Am. J. Phys. 57.(1989) 117.

[68] D. Twerenbold, Rep. Progr. Phys. 59 (1996) 239.

[69] W. Thirring, Lehrbuch der Mathematischen Physik, Springer, Berlin, 1980.

[70] D. Wallace, The quantum measurement problem: state of play, arXiv:0712.0149v1 [quant-ph].

[71] A. Wehrl, Rev. Mod. Phys. 50 (1978) 221. 
[72] S. Weinberg, The Quantum Theory of Fields Vol. I, Chap. 4. Cambridge University Press, Cambridge 1995.

[73] E. P. Wigner, Group Theory, Academic Press, New York, 1959.

[74] F. Wilczek, Phys. Rev. Letters 49 (1982) 957.

[75] D. Giulini, E. Joos, C. Kiefer, J. Kupsch, I.-O. Stamatescu, H. D. Zeh, Decoherence and the Appearance of Classical World in Quantum Theory, Springer, Berlin, 1996.

[76] W. H. Zurek, Rev. Mod. Phys.,75 (2003) 715. 